\title{
Unification with vector-like fermions and signals at LHC
}

\author{
Biplob Bhattacherjee, ${ }^{a}$ Pritibhajan Byakti, $^{b}$ Ashwani Kushwaha ${ }^{a}$ and \\ Sudhir K. Vempati ${ }^{a}$ \\ ${ }^{a}$ Centre for High Energy Physics, Indian Institute of Science, \\ Bangalore 560012, India \\ ${ }^{b}$ Department of Physics, \\ Pandit Deendayal Upadhyaya Adarsha Mahavidyalaya (PDUAM) Eraligool, \\ Karimganj, 788723, Assam, India \\ E-mail: biplob@iisc.ac.in, priti137@gmail.com, ashwanik@iisc.ac.in, \\ vempati@iisc.ac.in
}

ABStract: We look for minimal extensions of Standard Model with vector like fermions leading to precision unification of gauge couplings. Constraints from proton decay, Higgs stability and perturbativity are considered. The simplest models contain several copies of vector fermions in two different (incomplete) representations. Some of these models encompass Type III seesaw mechanism for neutrino masses whereas some others have a dark matter candidate. In all the models, at least one of the candidates has non-trivial

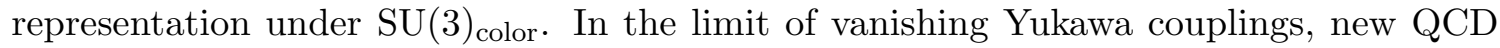
bound states are formed, which can be probed at LHC. The present limits based on results from $13 \mathrm{TeV}$ already probe these particles for masses around a TeV. Similar models can be constructed with three or four vector representations, examples of which are presented.

KEYwords: Beyond Standard Model, GUT

ARXIV EPRINT: 1702.06417v2 


\section{Contents}

1 Introduction 1

2 Recap of essential RG 3

2.1 One loop gauge unification 3

2.2 Two loop RG evolution of gauge couplings 5

2.3 Evolution of Higgs self coupling $\quad 6$

3 Gauge coupling unification with vector-like fermions $\quad 8$

4 Minimal unificon models $\quad 10$

$\begin{array}{llr}4.1 & \text { Model } 1 & 10\end{array}$

$\begin{array}{llr}4.2 & \text { Model } 2 & 13\end{array}$

$\begin{array}{llr}4.3 & \text { Model } 3 & 14\end{array}$

$\begin{array}{lll}4.4 & \text { Model } 4 & 17\end{array}$

$\begin{array}{llr}4.5 & \text { Model } 5 & 19\end{array}$

$\begin{array}{lll}4.6 & \text { Model } 6 & 20\end{array}$

$\begin{array}{lll}4.7 & \text { Model } 7 & 22\end{array}$

4.8 Model $8 \quad 24$

$\begin{array}{lll}4.9 & \text { Model } 9 & 26\end{array}$

5 Collider signature of minimal vector-like fermion models 28

5.1 Decay operators 28

$\begin{array}{lll}5.2 & \text { Formalism for bound state } & 29\end{array}$

$\begin{array}{lll}5.3 & \text { Signals } & 30\end{array}$

5.3.1 $\gamma \gamma, Z Z, Z \gamma, W^{+} W^{-}$channel $\quad 30$

5.3.2 Dijet channel 31

5.4 Limits on signals from CMS and ATLAS 31

5.4.1 Dijet bounds 34

5.4.2 Diphoton bounds 34

6 Summary and outlook $\quad 35$

$\begin{array}{ll}\text { A Two representation case } & 36\end{array}$

$\begin{array}{ll}\text { B Three representation case } & 36\end{array}$

$\begin{array}{ll}\text { C Four representation case } & 37\end{array}$

$\begin{array}{ll}\text { D Representations and Dynkin indices } & 37\end{array}$ 
E Mixing between SM particle with vector-like fermion

$\begin{array}{lll}\text { E.1 Vector like quarks } & 37\end{array}$

E.2 Vector like leptons 41

F Earlier scan of models by Tom Rizzo 42

G Two loop beta function $\quad 43$

\section{Introduction}

For the past few decades, the path to Beyond Standard Model (BSM) physics has been dictated mostly by solutions to the hierarchy problem [1]. However, with no experimental evidence to support this endeavor, from either LEP, Tevatron or the LHC so far, one might wish to explore alternate paths which do not contain a solution to the hierarchy problem. Furthermore, there could solutions to the hierarchy problem which do not introduce any new particles all the way up to GUT scales. The relaxion idea and its variant for example, propose a cosmological solution to the hierarchy problem without introducing any new physics at the weak scale $[2-5]$.

One of the guiding principles for these alternate paths is the unification of gauge coupling constants. Popular models like split supersymmetry [6-8] have been proposed which have part of the MSSM particle spectrum at the weak scale and rest (scalar spectrum) at an intermediate scale. The current limits on the stable, long lived R-hadrons which are a prediction of these models are about $1.5-1.61 \mathrm{TeV}[9,10]$. However, this framework depends crucially on the underlying MSSM framework. Generalization without supersymmetry are important to explore.

With this view point, we revisit extensions of the Standard Model with vector-like fermions which lead to precision gauge coupling unification (for earlier works in this direction, please see [11-32]). There are several virtues of these models:

(i) They have minimal constraints from electroweak precision parameters, especially from S and T parameters [33-39], as long as the mixing between vector-like fermions and SM fermions is small.

(ii) They do not lead to any anomalies as they are vector in nature.

(iii) They can be tested directly at the collider experiments like LHC. The kind of signals depend whether on the amount of mixing they have with the Standard Model fields.

(iv) If they have mixing with SM quarks, it is possible that they can be probed indirectly in flavour physics.

To our knowledge, there has not been a recent survey of models containing vector fermions leading to gauge coupling unification. An earlier analysis was done in ref. [13] with the available LEP data at that time. We have updated where those models stand in the appendix, F. In addition to improvements in the gauge coupling measurements and 
theoretical threshold calculations which are now available at NNLO, an important role is played by the experimental discovery and the (almost) precise determination of the Higgs mass. It has been shown that the Higgs potential becomes unstable from scales close to $10^{11} \mathrm{GeV}$ [40], depending on the exact values of the top mass and alpha $a_{s}$. Thus a Grand Unified Theory should not only lead to gauge coupling unification but also keep Higgs potential stable all the way up to the GUT scale.

In the models presented here as we will see the Higgs potential naturally remains stable all the way up to the GUT scale. In the view that the primary existence of these vector particles is unification of gauge couplings, we dub them "unificons". However, as we will see later, these models do not restrict themselves only to unification. In some models, we find solutions with a provision for Type III seesaw mechanism for neutrino masses, and in some others there is a WIMP (Weakly Interacting Massive Particle) dark matter candidate. Thus "unificon" models can indeed have wide phenomenological reach solving other problems in Standard Model like neutrino masses and dark matter.

As a search for all possible models with extra vector-like fermions would be a herculean task, we resort to minimality. We assume unification of gauge couplings á la SU(5). Additional vector-like particles appear as incomplete representations of SU(5). We have looked at all possible incomplete decompositions emanating from $\mathrm{SU}(5)$ representations up to dimension 75. The number of copies in each representation is taken to be $n$ which is an integer between 1 and 6 . The mass range of these additional vector-like fermions is chosen to be $m \sim k \mathrm{TeV}$, where $k$ is a $\mathcal{O}(1)$ number taken to be approximately between $1 / 4$ to 5 .

There are no solutions with successful gauge coupling unification as long as the vectorlike fermions come in one single representation. This holds true even if increase the number of copies all the way to six, the maximum we have allowed per representation. ${ }^{1}$ The minimal set of successful models with two different representations each with varied number of copies is listed in table 2. All these models satisfy constraints from proton decay and the stability of the Higgs potential. Both representations come in several copies. Some solution allows for degeneracy between the fermions of the different representations, where as in some cases require non-degeneracy of the fermions in representation 1 and fermions in representation 2 .

Interestingly all models have at least one representation with non trivial colour quantum numbers which makes them attractive from LHC point of view. In the limit of negligible Yukawa couplings, these colour states in $\mathrm{SU}(3)$ representations of the type 3, 6, 8 form bound states and are produced at LHC. The present limits on these bound states from $13 \mathrm{TeV}$ run of LHC are already touching the $1 \mathrm{TeV}$ mark, depending on the decay mode and the final states. We provide in detail limits on the relevant $\mathrm{SU}(3)$ representation bound states.

We also looked for solutions with three and four different representations. Unlike the two representation case, we considered degenerate spectrum for all the vector-like fermions in these two cases. Several solutions are found which are listed in appendix B and appendix C. The rest of the paper is organised as follows: in the next section 2 we

\footnotetext{
${ }^{1}$ We have considered the Yukawa couplings of the extra vector-like fermions and the mixing with the SM fermions to be negligible. This can be arranged by imposing discrete symmetry.
} 
recap the essential $\mathrm{RG}$ required for gauge coupling unification and stability of the Higgs potential. In section 3 we present the results for two fermion different representation case. In section 4 we present the properties of each successful model. In section 5 , we discuss the bound state formalism of the colour vector-like fermions and limit from LHC. We close with a conclusion and outlook. In appendix $\mathrm{D}$ we have tabulated all forty representations of $\mathrm{SU}(3) \times \mathrm{SU}(2) \times \mathrm{U}(1)$ coming from $\mathrm{SU}(5)$ representations upto dimension 75 [41] with their Dynkin index. In appendix E constraints on mixing between SM fermions with vector-like quark is summarized. Appendix G summaries the two-loop RG equation of Standard Model.

\section{Recap of essential RG}

\subsection{One loop gauge unification}

It is well known that gauge couplings do not unify precisely in the Standard Model. If one insists on unification of the guage couplings at the GUT scale, the required $\sin ^{2} \theta_{W}\left(M_{Z}^{2}\right)$ is 0.204 (for one loop beta functions) instead of the current experimental value of $\sin ^{2} \theta_{W}\left(M_{Z}^{2}\right)$ $=0.23129 \pm 0.00005$ [42]. As argued in the introduction, in the present work, we look for additional vector-like matter fermions, close to the weak scale, which can compensate the deviation and lead to successful gauge coupling unification. At the 1-loop level, the beta functions for the three gauge couplings are given as

$$
\frac{d g_{l}}{d t}=-\frac{1}{16 \pi^{2}} b_{l} g_{l}^{3}, \quad \text { where } \quad t=\ln \mu,
$$

where is $l=\{\mathrm{U}(1), \mathrm{SU}(2), \mathrm{SU}(3)\}$ runs over all the three gauge groups. The $b_{l}$ functions have the general form:

$$
b_{l}=\left[\frac{11}{3} C\left(V_{l}\right)-\frac{2}{3} T\left(F_{l}\right)-\frac{1}{3} T\left(S_{l}\right)\right] .
$$

Here $C(R)$ is quadratic Casimir and $T(R)$ is Dynkin index of representation R. $V, F$ and $S$ represents vector, Weyl fermion and complex scalar field respectively. For U(1) group $T\left(R_{1}\right)$ and $C\left(R_{1}\right)$ are

$$
T\left(R_{1}\right)=C\left(R_{1}\right)=\frac{3}{5} Y^{2} .
$$

For $\mathrm{SU}(\mathrm{N})$ group $T(R)$ is defined as follows:

$$
\operatorname{Tr}\left[R^{i} R^{j}\right]=T(R) \delta^{i j} .
$$

The following are the list of Dynkin Indices for lower dimensional Representations:

\begin{tabular}{|l|c|}
\hline Representation & $T(N)$ \\
\hline Fundamental & $\frac{1}{2}$ \\
Adjoint & $n$ \\
Second Rank anti-symmetric tensor & $\frac{n-2}{2}$ \\
Second Rank symmetric tensor & $\frac{n+2}{2}$ \\
\hline
\end{tabular}


More complete list on quadratic casimirs can be found in [43]. Within SM, the beta functions take the value

$$
b_{1}^{0}=-\frac{41}{10}, b_{2}^{0}=\frac{19}{6}, \text { and } b_{3}^{0}=7 .
$$

In the presence of a vector-like fermion $V_{1}$ at the scale $M_{1}$ greater than weak scale, given the gauge coupling unification at $M_{\mathrm{GUT}}$, the (eq. (2.1)) take the form:

$$
\alpha_{l}^{-1}\left(\mu_{\mathrm{in}}\right)=\frac{b_{l}^{0}}{2 \pi} \ln \frac{\mu_{\mathrm{in}}}{M_{\mathrm{GUT}}}+\frac{b_{l}^{\mathrm{V}_{1}}}{2 \pi} \ln \frac{M_{1}}{M_{\mathrm{GUT}}}+\alpha_{l}^{-1}\left(M_{\mathrm{GUT}}\right)
$$

where $\alpha_{l}=\frac{g_{l}^{2}}{4 \pi}$ and $b_{l}^{\mathrm{V}_{1}}$ capture effect of addition of vector-like fermions at the scale $M_{1}$. The parameter $\bar{b}$ is an useful measure of unification of gauge couplings. It is defined as

$$
\begin{aligned}
\bar{b}\left(\mu_{\mathrm{in}}\right)= & \frac{\alpha_{3}^{-1}\left(\mu_{\mathrm{in}}\right)-\alpha_{2}^{-1}\left(\mu_{\mathrm{in}}\right)}{\alpha_{2}^{-1}\left(\mu_{\mathrm{in}}\right)-\alpha_{1}^{-1}\left(\mu_{\mathrm{in}}\right)} \\
= & \frac{\triangle b_{32}^{0}+\left(\triangle b_{32}^{\mathrm{V}_{1}}\right) \ln \left(M_{1} / M_{\mathrm{GUT}}\right) / \ln \left(\mu_{\mathrm{in}} / M_{\mathrm{GUT}}\right)}{\triangle b_{21}^{0}+\left(\triangle b_{21}^{\mathrm{V}_{1}}\right) \ln \left(M_{1} / M_{\mathrm{GUT}}\right) / \ln \left(\mu_{\mathrm{in}} / M_{\mathrm{GUT}}\right)} .
\end{aligned}
$$

Where the second line can be derived from eq. (2.6) assuming unification at $M_{\mathrm{GUT}}$. The parameters $\Delta b_{l k}$ are defined as $b_{l}-b_{k}$. In the absence of new vector-like particles, $\bar{b}$ is independent of the running scale $\mu$. In their presence however, there is a $\mu$ dependence but it is typically mild. For the case where the new particles are close to weak scale $\sim \mathrm{TeV}$, and when $\mu_{\text {in }}=M_{Z}$, the $\log$ factor, $\ln \left(M_{1} / M_{\mathrm{GUT}}\right) / \ln \left(\mu / M_{\mathrm{GUT}}\right)$ is close to one. In this case, the expression for unified theories is given by

$$
\bar{b}=\frac{\triangle b_{32}^{0}+\triangle b_{32}^{V_{1}}}{\triangle b_{21}^{0}+\triangle b_{21}^{V_{1}}}
$$

Note that the eq. (2.7) can purely be determined from experiments at $M_{Z}$. It's value is given by

$$
\bar{b}\left(M_{Z}\right)=0.718
$$

In the SM, if we insist on unified gauge couplings at $M_{\mathrm{GUT}}$, at the weak scale, $\bar{b}$ takes the value 0.5 clearly in conflict with experiments. In MSSM, $\bar{b}$ turns out to be $5 / 7$. Of course, these arguments are valid only at one loop. There is deviation in eq. (2.9) when higher loops are considered. In our analysis, most of the successful models have a $\bar{b}$ of 0.67 to 0.833 . The above discussion can be easily generalised for more than one Vector field $V_{i}$ at scales $M_{i}$. It has the following general form at the 1-loop level.

$$
\bar{b}(\mu)=\frac{\Delta b_{32}^{0}+\sum_{i}\left(\Delta b_{32}^{\mathrm{V}_{\mathrm{i}}} \ln \left(M_{i} / M_{\mathrm{GUT}}\right)\right) / \ln \left(\mu / M_{\mathrm{GUT}}\right)}{\Delta b_{21}^{0}+\sum_{i}\left(\Delta b_{21}^{\mathrm{V}_{\mathrm{i}}} \ln \left(M_{i} / M_{\mathrm{GUT}}\right)\right) / \ln \left(\mu / M_{\mathrm{GUT}}\right)},
$$

where we assumed the hierarchy of the scales as $M_{1}<M_{2}<M_{3}$ etc. 


\subsection{Two loop RG evolution of gauge couplings}

To improve the precision in unification of gauge couplings, we consider two loop beta functions. At the two loop level, the beta functions involve Yukawa couplings which makes them model dependent. Vector-like fermions which typically have "bare" mass terms in the Lagrangian, can also mix with the Standard Model fermions through Yukawa interactions if allowed by the gauge symmetry. However, this mixing is subject to strong phenomenological constraints [33-37]. A detailed Discussion on the mixing constraints can be found in appendix E.

In the present analysis, we restrict ourselves to models with minimal or zero vector-like fermion and SM mixing through the Higgs mechanism. With this assumption, we can safely neglect the Yukawa contribution from the new sector to the gauge coupling unification. The RG equations at the two loop level are given by [44-46]:

$$
\frac{d g_{l}}{d t}=-b_{l} \frac{g_{l}^{3}}{16 \pi^{2}}-\sum_{k} m_{l k} \frac{g_{l}^{3} g_{k}^{2}}{\left(16 \pi^{2}\right)^{2}}-\frac{g_{l}^{3}}{\left(16 \pi^{2}\right)^{2}} \operatorname{Tr}\left\{C_{l u} Y_{u}^{\dagger} Y_{u}+C_{l d} Y_{d}^{\dagger} Y_{d}+C_{l e} Y_{e}^{\dagger} Y_{e}\right\},
$$

where the first term in the right hand side is due to one-loop which was discussed in the previous subsection. The second term is purely from gauge interactions whereas the third terms involves the Yukawa terms $Y_{u, d, e}$ where the suffixes mean the up-type,down-type and lepton-type couplings. The expression for the coefficients appearing in the second term of the above equation are as follows [44, 47]:

$$
\begin{aligned}
m_{l k}= & \left(2 C\left(F_{k}\right) d\left(F_{k}\right) T\left(F_{l}\right) d\left(F_{m}\right)+4 C\left(S_{k}\right) d\left(S_{k}\right) T\left(S_{k}\right) d\left(S_{m}\right)\right) \quad \text { where } \quad l \neq k \\
m_{l l}= & {\left[\frac{10}{3} C\left(V_{l}\right)+2 C\left(F_{l}\right)\right] T\left(F_{l}\right) d\left(F_{m}\right) d\left(F_{k}\right)+\left[\frac{2}{3} C\left(V_{l}\right)+4 C\left(S_{l}\right)\right] T\left(S_{l}\right) d\left(S_{m}\right) d\left(S_{k}\right) } \\
& -\frac{34}{3}\left[C\left(V_{l}\right)^{2}\right],
\end{aligned}
$$

where $d(R)$ means dimension of the representation $R$ and other factors $\mathrm{C}(\mathrm{R})$ and $\mathrm{T}(\mathrm{R})$ are already defined in eq. (2.2).

For the Standard Model, the values of $m_{l k}$ are as follows:

$$
m^{0}=-\left(\begin{array}{ccc}
\frac{199}{50} & \frac{27}{10} & \frac{44}{5} \\
\frac{9}{10} & \frac{35}{6} & 12 \\
\frac{11}{10} & \frac{9}{2} & -26
\end{array}\right) .
$$

In the third term of eq. (2.12), we have the coefficients $C_{l f}$ and for the standard model particles it has the following form:

$$
C^{0}=\left(\begin{array}{ccc}
\frac{17}{10} & \frac{1}{2} & \frac{3}{2} \\
\frac{3}{2} & \frac{3}{2} & \frac{1}{2} \\
2 & 2 & 0
\end{array}\right)
$$

As we are considering the Yukawa couplings between the vector-like fermions with Higgs boson to be negligible, ${ }^{2}$ the contribution of vector-like particles to $C_{l f}$ coefficient can be

\footnotetext{
${ }^{2}$ This can be organised by imposing discrete symmetries distinguishing SM partners from vectorlike fermions.
} 


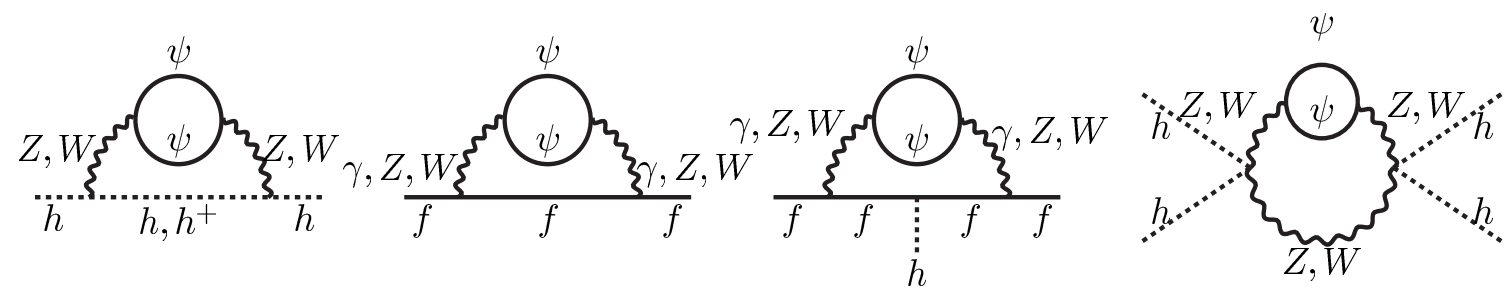

Figure 1. Diagrams Contributing in Two loop RG of Yukawa and Higgs quartic couplings, through new Fermion fields $(\psi)$. Here $f$ is any standard model fermion. First two diagrams correspond to anomalous dimension and the last two diagrams are giving vertex corrections.

taken as zero. On the other hand $\delta m_{i j} \neq 0$, where $\delta$ is used to indicate contribution from additional vector-like fermions. We'll give explicit values of $\delta m_{i j}$ for each of the viable models in section 4 .

Two-loop RG running for the Yukawa couplings is given as

$$
Y_{u, d, e}^{-1} \frac{d Y_{u, d, e}}{d t}=\frac{1}{16 \pi^{2}} \beta_{u, d, e}^{(1) \mathrm{SM}}+\frac{1}{\left(16 \pi^{2}\right)^{2}} \beta_{u, d, e}^{(2) \mathrm{SM}}
$$

The SM RG for these Yukawa couplings are shown in appendix G. Here we will address the effect of new fermion fields in RG of Yukawa couplings [47-50]. The one loop beta functions of these couplings are not be affected by new matter(fermion) fields because we considered the Yukawa couplings between the vector-like fermions with Higgs boson to be negligible. Two loop beta functions get contributions from the diagrams shown in figure 1 , which results in the following terms:

$$
\begin{aligned}
\delta \beta_{u}^{(2) V} & =\frac{40}{9} g_{3}^{4} T\left(F_{3}\right) d\left(F_{2}\right) d\left(F_{1}\right)+\frac{29}{90} g_{1}^{4} T\left(F_{1}\right) d\left(F_{3}\right) d\left(F_{2}\right)+\frac{1}{2} g_{2}^{4} T\left(F_{2}\right) d\left(F_{3}\right) d\left(F_{1}\right) \\
\delta \beta_{d}^{(2) V} & =\frac{40}{9} g_{3}^{4} T\left(F_{3}\right) d\left(F_{2}\right) d\left(F_{1}\right)-\frac{1}{90} g_{1}^{4} T\left(F_{1}\right) d\left(F_{3}\right) d\left(F_{2}\right)+\frac{1}{2} g_{2}^{4} T\left(F_{2}\right) d\left(F_{3}\right) d\left(F_{1}\right) \\
\delta \beta_{e}^{(2) V} & =\frac{11}{10} g_{1}^{4} T\left(F_{1}\right) d\left(F_{3}\right) d\left(F_{2}\right)+\frac{1}{2} g_{2}^{4} T\left(F_{2}\right) d\left(F_{3}\right) d\left(F_{1}\right)
\end{aligned}
$$

\subsection{Evolution of Higgs self coupling}

The modification of the gauge beta functions in the presence of additional vector-like particles can have implications on the evolution of the Higgs self coupling. At the outset, one might consider that since there are no new large Yukawa couplings, ${ }^{3}$ the evolution of the Higgs self coupling might be in the safe region. While this is true, the evolution of the SM Yukawa couplings is itself modified in these models as seen in the previous sub-section. It is thus worthwhile to check explicitly the stability of Higgs self coupling along with gauge coupling unification.

To check the Higgs stability we follow [40, 51, 52] who have checked for the stability using three loop beta functions and NNLO matching conditions. We use the beta function of

\footnotetext{
${ }^{3}$ Firstly by assumption, As we will see in the next section, this is automatic in most models as Yukawa couplings with new vector-like fermions are not gauge invariant.
} 


\begin{tabular}{|ccc|}
\hline Parameter & Value & Description \\
\hline$M_{W}$ & $80.384 \pm 0.015 \mathrm{GeV}$ & Pole mass of W boson [53] \\
$M_{Z}$ & $91.1876 \pm 0.0021 \mathrm{GeV}$ & Pole mass of Z boson [53] \\
$M_{h}$ & $125.09 \pm 0.21 \pm 0.11 \mathrm{GeV}$ & Pole mass of Higgs boson [54] \\
$M_{t}$ & $173.34 \pm 0.76 \pm 0.3 \mathrm{GeV}$ & Pole mass of top quark [55] \\
$\alpha_{3}\left(M_{Z}\right)$ & $0.1184 \pm 0.0007$ & $\overline{M S}$ gauge SU $(3)_{c}$ coupling [56] \\
\hline
\end{tabular}

Table 1. Input values of SM observales used to fix the SM fundamental parameters.

the $\lambda$ at the two loop and put a condition that $\lambda$ is always positive at all scales of evolution. Two-loop RG running for the Higgs quartic coupling are shown below

$$
\frac{d \lambda}{d t}=\frac{1}{16 \pi^{2}} \beta_{\lambda}^{(1) \mathrm{SM}}+\frac{1}{\left(16 \pi^{2}\right)^{2}} \beta_{\lambda}^{(2) \mathrm{SM}}
$$

where beta functions for SM Higgs quartic couplings are defined in appendix G. The effect of new fermion fields in RG of Higgs quartic couplings are:

$$
\begin{aligned}
\delta \beta_{\lambda}^{(2) V}= & -\frac{1}{25} g_{1}^{4}\left(12 g_{1}^{2}+20 g_{2}^{2}-25 \lambda\right) T\left(F_{1}\right) d\left(F_{3}\right) d\left(F_{2}\right) \\
& -\frac{1}{5} g_{2}^{4}\left(4 g_{1}^{2}+20 g_{2}^{2}-25 \lambda\right) T\left(F_{2}\right) d\left(F_{3}\right) d\left(F_{1}\right)
\end{aligned}
$$

To solve the RG equations we need boundary values of the coupling constants and masses at the top mass $\left(M_{t}\right)$ scale. The quantities of interest are Higgs quartic coupling $(\lambda)$, Yukawa couplings and gauge coupling, which can be calculated in terms of physical observables W-boson mass $\left(M_{W}\right)$, Z-boson mass $\left(M_{Z}\right)$, Higgs mass $\left(M_{h}\right)$ and $\alpha_{3}\left(M_{Z}\right)$ at the two loop level. The input parameters are calculated in the $\overline{M S}$-scheme. More detailed can be found in [52]. For the RG running we use the central value of Top mass. The input values of SM parameters and couplings are listed in table 1.

Values of the relevant couplings at scale $M_{t}$ are as follows:

$$
\begin{aligned}
\frac{\lambda\left(M_{t}\right)}{2} & =0.12604+0.00206\left(M_{h}-125.15\right)-0.00004\left(M_{t}-173.34\right) \pm 0.00030_{t h} \\
y_{t}\left(M_{t}\right) & =0.93690+0.00556\left(M_{t}-173.34\right)-0.00042\left(\alpha_{3}\left(M_{Z}\right)-0.1184\right) / 0.0007, \\
g_{2}\left(M_{t}\right) & =0.64779+0.00004\left(M_{t}-173.34\right)+0.00011 \frac{M_{W}-80.384}{0.014} \\
g_{Y}\left(M_{t}\right) & =0.35830+0.00011\left(M_{t}-173.34\right)+0.00020 \frac{M_{W}-80.384}{0.014} \\
g_{3}\left(M_{t}\right) & =1.1666+0.00314 \frac{\alpha_{3}\left(M_{Z}\right)-0.1184}{0.0007}-0.00046\left(M_{t}-173.34\right)
\end{aligned}
$$

where all the parameters with mass dimension has written in $\mathrm{GeV}$. Central values of the above couplings are calculated upto NNLO ([40] for $\lambda$ ) order for all of them except the $y_{t}\left(M_{t}\right)$ for which we considered NNNLO [57-59]. The value of $\alpha_{3}\left(M_{Z}\right)$, is extracted from the global fit of ref. [56] in the effective SM with 5 flavours. Including RG running from $M_{Z}$ to $M_{t}$ at 4 loops in QCD and at 2 loops in the electroweak gauge interactions, and 
3 loop QCD matching at $M_{t}$ to the full SM with 6 flavours, the strong gauge couplig is calculated. The contribution of the bottom and tau Yukawa couplings, are computed from the $\overline{M S}$ b-quark mass $\left(M_{b}\left(M_{t}\right)=2.75 \mathrm{GeV}\right)$ and Tau mass $\left(M_{\tau}\left(M_{t}\right)=1.742 \mathrm{GeV}\right)[60]$.

Threshold corrections at GUT scale. One of the main concerns which remains now is the possible effect of threshold corrections at the GUT scale, which can be quite significant. These corrections are highly model dependent. In some GUT models, with no extra matter at the weak scale (other than the Standard Model particle content), it is possible to achieve gauge coupling unification through large threshold corrections at the GUT scale [61]. While such extreme situations are no longer valid due to the constraint on the stability of the Standard Model Higgs potential, it is still possible that GUT scale threshold corrections could play an important role. To study the impact of threshold corrections on gauge coupling unification, we define the following parameters: $\alpha_{\text {ave. }}(\mu)=\left(\alpha_{1}(\mu)+\alpha_{2}(\mu)+\alpha_{3}(\mu)\right) / 3$ and $\bar{\triangle}_{i}(\mu)=\left(\alpha_{i}(\mu)-\alpha_{\text {avg }}(\mu)\right) / \alpha_{\text {ave }}(\mu)$. Note that $\alpha_{\text {ave }}$ coincides with $\alpha_{\mathrm{GUT}}$ when all $\bar{\triangle}_{i} \rightarrow 0$, at the scale $M_{\mathrm{GUT}}$. In the presence of threshold corrections, one could allow for deviations in $\alpha_{\mathrm{GUT}}$ in terms of $\bar{\triangle}_{i}$ at the GUT scale ${ }^{4}$ Defining $\triangle=\max \left(\triangle_{i}\right)$, we see that $\triangle$ is as large as $6 \%$ in the Standard Model. In our survey of models below, we have allowed for variations in $\triangle$ up to $1.2 \%$. A more conservative set of models is tabulated in appendix A which have $\triangle$ of $3 \%$.

Proton decay. Models studied in this work can lead to proton decay mediated by the gauge bosons at GUT scale. The lifetime of proton decay is extremely sensitive to the heavy gauge bosons $\left(M_{(X, Y)} \sim M_{\mathrm{GUT}}\right)$. For these models, using the simple decay width formulae, $\Gamma \sim \alpha_{\text {Gut }} \frac{m_{\text {proton }}^{5}}{M_{\text {GUT }}^{4}}$ we estimate the life time of the proton, where the current experimental value is of order $>10^{32}-10^{34}$ years [63].

\section{Gauge coupling unification with vector-like fermions}

As mentioned in Introduction, in our search for successful models with gauge coupling unification, we focus on vector-like matter in incomplete representations of SU(5). We have considered (incomplete) representations [41] up-to dimension 75, which contains a 15 of $\mathrm{SU}(3)$ of QCD as the largest component. The full list of incomplete representations is presented in appendix D. As can be seen from the table 8, there are 40 representations which we have considered. Note that representations 4,5 in table 8 do not come as incomplete representations of $\mathrm{SU}(5)$ instead they are singlet representations of $\mathrm{SU}(5)$. Our search strategy is start with $n_{i}$ copies of representation $i$, with all the $n_{i}$ copies degenerate in mass, $m_{i}$ and look for unification of the gauge couplings. The maximum number of copies is taken to be 10 . The number of representation types $i$ considered simultaneously is restricted up to four. An important constraint comes from proton decay, which restricts the scale of unification to lie above (at least) $10^{15} \mathrm{GeV}$. As mentioned above, in addition to unification, we also consider that the Higgs potential should be stable all the way up to the GUT scale.

\footnotetext{
${ }^{4}$ Another model independent parameterisation for the threshold corrections was presented in [62].
} 


\begin{tabular}{|c|c|c||c|c||c|c|c|c|c|}
\hline $\begin{array}{c}\text { Mod } \\
\text { No. }\end{array}$ & Rep 1 & $\begin{array}{c}M_{\text {Rep1 }} \\
\text { GeV }\end{array}$ & Rep 2 & $\begin{array}{c}M_{\text {Rep2 }} \\
\text { GeV }\end{array}$ & $\begin{array}{c}\text { One } \\
\text { loop }\end{array}$ & $\begin{array}{c}\text { Two } \\
\text { loop }\end{array}$ & $\begin{array}{c}\text { Vaccum } \\
\text { Stability }\end{array}$ & $\begin{array}{c}M_{\text {GUT }} \\
\times 10^{16} \mathrm{GeV}\end{array}$ & $\alpha_{\text {GUT }}$ \\
\hline 1 & $6\left(1,2, \frac{1}{2}\right)$ & $(250-5000)$ & $1\left(6,1, \frac{1}{3}\right)$ & $(250-5000)$ & $\checkmark$ & $\checkmark$ & $\checkmark$ & $\sim 0.11$ & $\sim 0.038$ \\
\hline 2 & $6\left(1,2, \frac{1}{2}\right)$ & $(250-2000)$ & $2(8,1,0)$ & $(500-5000)$ & $\checkmark$ & $\checkmark$ & $\checkmark$ & $\sim 2.34$ & $\sim 0.040$ \\
\hline 3 & $2(1,3,0)$ & $(250-5000)$ & $4\left(3,1, \frac{1}{3}\right)$ & $(250-5000)$ & $\checkmark$ & $\checkmark$ & $\checkmark$ & $\sim 2.29$ & $\sim 0.030$ \\
\hline 4 & $2\left(3,1, \frac{2}{3}\right)$ & $(250-5000)$ & $2\left(3,2, \frac{1}{6}\right)$ & $(250-4500)$ & $\checkmark$ & $\checkmark$ & $\checkmark$ & $\sim 4.79$ & $\sim 0.040$ \\
\hline 5 & $3(1,3,0)$ & $(1800-5000)$ & $1\left(6,1, \frac{2}{3}\right)$ & $(250-950)$ & $\checkmark$ & $\checkmark$ & $\checkmark$ & $\sim 1.08$ & $\sim 0.037$ \\
\hline 6 & $1\left(1,4, \frac{1}{2}\right)$ & $(250-2000)$ & $2\left(6,1, \frac{2}{3}\right)$ & $(1000-5000)$ & $\checkmark$ & $\checkmark$ & $\checkmark$ & $\sim 8.58$ & $\sim 0.107$ \\
\hline 7 & $1\left(3,1, \frac{1}{3}\right)$ & $(250-5000)$ & $1\left(3,2, \frac{1}{6}\right)$ & $(250-5000)$ & $\checkmark$ & $\checkmark$ & $\checkmark$ & $\sim 2.20$ & $\sim 0.028$ \\
\hline 8 & $4\left(1,2, \frac{1}{2}\right)$ & $(300-5000)$ & $1(8,1,0)$ & $(300-5000)$ & $\checkmark$ & $\checkmark$ & $\checkmark$ & $\sim 0.10$ & $\sim 0.030$ \\
\hline 9 & $3(1,3,0)$ & $(1100-5000)$ & $6\left(3,1, \frac{1}{3}\right)$ & $(250-1800)$ & $\checkmark$ & $\checkmark$ & $\checkmark$ & $\sim 25.0$ & $\sim 0.037$ \\
\hline
\end{tabular}

Table 2. Model with two vector-like fermions representation satisfying gauge coupling unification and vacuum stability condition, considering $\triangle$ of $1.2 \%$.

In the computations, we have also varied the input parameters to lie within their two sigma regions. The masses of new vector-like are assumed to lie between $250 \mathrm{GeV}-5 \mathrm{TeV}$.

For $i=1$ we searched for the mass of the vector-like fermion from $250 \mathrm{GeV}-5 \mathrm{TeV}$, considering number of vector-like fermions $n_{1}=6$. These masses for $n_{1}$ copies have been considered degenerate for simplicity and no successful model was observed. The simplest solutions we found contain at least two different representation content each with a different number of copies. We call these solutions "minimal unificon models". These are listed in table 2. We now explain the notation used in the table. The two representations considered are called Rep1 and Rep2. The representation is described as $n_{i}\left(R_{\mathrm{SU}(3)}, R_{\mathrm{SU}(2)}, R_{\mathrm{U}(1)}\right)$, where $n_{i}$ introduced earlier is the number of copies of the representation, $R_{G}$ is the representation of the field under the gauge group G of the SM.

Furthermore, in the above, we mentioned only one part of the representation instead of the complete vector multiplet for brevity. For example, $\left(1,2, \frac{1}{2}\right)$ actually means $\left(1,2, \frac{1}{2}\right) \oplus$ $\left(1,2,-\frac{1}{2}\right)$. Colored representations like $\left(3,1, \frac{2}{3}\right)$ may mean two possibilities: (a) $\left(3,1, \frac{2}{3}\right) \oplus$ $\left(\overline{3}, 1,-\frac{2}{3}\right)$ and $(\mathrm{b})\left(\overline{3}, 1, \frac{2}{3}\right) \oplus\left(3,1,-\frac{2}{3}\right)$. On the other hand, the real representations like $(1,3,0)$ and $(8,1,0)$ are not short-hand notations. In the second last column, the entries are written in units of $10^{16} \mathrm{GeV}$. Thus except the first model, all the models have unification scale larger than $10^{16} \mathrm{GeV}$. All models appeared as the solution of one loop RG equation. In the third and fifth columns, we show the mass range of the vector-like fields. One can see that if we increase the mass of one representation, the mass of the other field also increases (as shown in figure 2(b), figure 3(b), figure 4(b), figure 5(b), figure 6(b), figure 7(b), figure 8(b), figure 9 (b) and figure 10(b)).

Solutions with three types of representations are also possible. These are listed in table 6 of appendix B. Here we made a restricted choice that all the representations and their copies are degenerate in mass of about $1 \mathrm{TeV}$. As can been seen from the table, the minimum number of extra vector-like fermions required is seven over the three representations, where 
as the maximum number is eighteen. All of them have unification scale less than $10^{16} \mathrm{GeV}$, which puts them at risk with Proton decay. The life time of the proton in these models is of order $\sim 10^{32}$ years which in contrast with the experimental value $>10^{32}-10^{34}$ years [63]. The maximum number of representations we have chosen simultaneously is four. Searching for models with different masses for each copy and each representation is computationally very intensive. Thus, we have considered all the four representations and their copies to be degenerate in mass at $1 \mathrm{TeV}$. The list of successful models is given in table 7 of appendix $\mathrm{C}$. The minimum number of vector-like particles required over all representations is five and the maximum is twenty. As with the three representation case, we find that the Unification scale is smaller than $10^{16} \mathrm{GeV}$ with the exception of one model (Model No 17 of table 7 ). As before from the arguments of Proton decay, these models can have potentially small proton life times in conflict with experiment. We do not address this issue here.

\section{Minimal unificon models}

In this section we concentrate on the Minimal vector-like fermion Unification Models. The list of such of models is given in table 2. Several interesting features are evident from the table 2 .

(a) Except for the first and eigth model, all the models have unification scale above $10^{16}$ and thus are safe with proton decay. (b) The minimalist model is model 7 , with only two vector-like fermions one with a mass range of $0.250-5 \mathrm{TeV}$ and another within a mass range of $250-5000 \mathrm{GeV}$. This model might have constraints from direct searches of vector-like quarks at LHC and elsewhere if there is significant mixing with SM particles. In its absence, as we assumed here, the bound will be different. We will discuss it in the next section. (c) The maximum number of vector-like fermions needed is nine in Model 9.

We now discuss each of these models in detail.

\subsection{Model 1}

In this model, ${ }^{5}$ we have six copies of $\left(1,2, \frac{1}{2}\right)$, which we called Rep1, with mass range between $250 \mathrm{GeV}$ to $5000 \mathrm{GeV}$ and one copy of $\left(6,1, \frac{1}{3}\right)$, called Rep2, with mass range from $250 \mathrm{GeV}$ to $5000 \mathrm{GeV}$. Rep1 field is lepton doublet like field and thus it can interact with right handed electron and the Higgs field through Yukawa interactions. This field mainly decays to gauge bosons like $Z$ boson and $W^{ \pm}$. For the sake of simplicity of the two loop gauge coupling RG running, we impose appropriate $Z_{N}$ symmetries to these fermion doublets. This symmetry cut-down all the Yukawa terms involving these fields at the renormalisable level and only gauge couplings are allowed. Lightest neutral component of these fermions can be a dark matter candidate. This type of dark matter is called inert fermion doublet dark matter $[65,66]$. Rep2 is more exotic and at the renormalisation level, it can interact with the gauge bosons only. It cannot decay to any standard model particles. Thus they form bound states. Phenomenology of this is studied in detail in the next section 5 .

\footnotetext{
${ }^{5}$ We have cross-checked our Two Loop RG equation of this model with the publicly code SARAH [64] for consistency.
} 


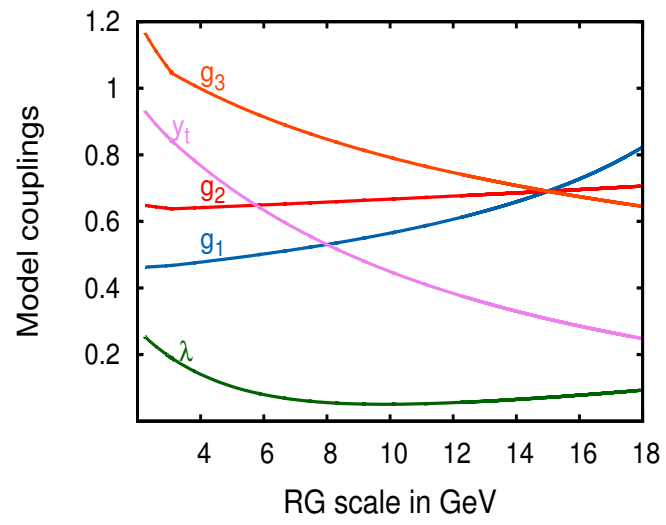

(a)

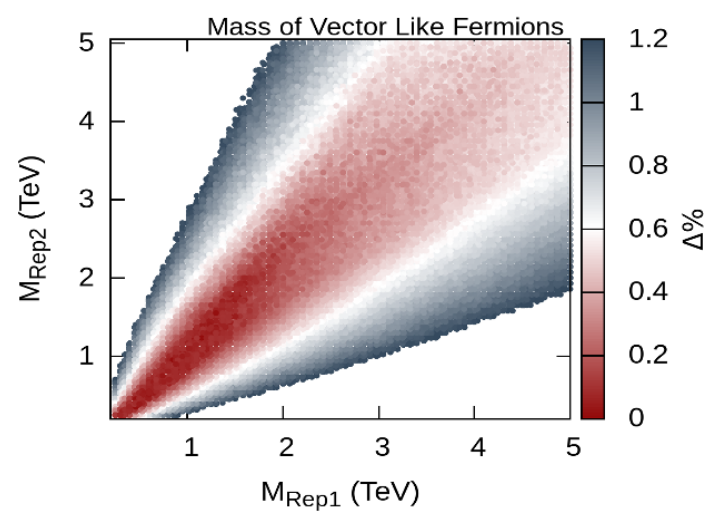

(b)

Figure 2. Model 1: figure (a) Gauge couplings $\left(g_{1}, g_{2}, g_{3}\right)$ unification and Vacuum stability $(\lambda>0)$ plot, considering vector-like fermion in Rep. 1 of mass $1210 \mathrm{GeV}$ and Rep. 2 of mass $1260 \mathrm{GeV}$. Figure (b) Mass range allowed for vector-like fermions in Rep.1 and Rep.2 for gauge unification and Vacuum stability.

For most points in this model vector-like fermions in Rep1 can be degenerate with vector-like fermions in Rep2 $\left(M_{\mathrm{Rep} 1} \sim M_{\mathrm{Rep} 2}\right)$ as shown in figure 2(b). However, there could be points in which either of the $M_{\mathrm{Rep} 1}>M_{\mathrm{Rep} 2}$ and $M_{\mathrm{Rep} 1}<M_{\mathrm{Rep} 2}$ are possible. The change in the beta functions in these three possibilities are as follows:

(a) $M_{\text {Rep1 }}=M_{\text {Rep2 }}$

(I) $\mu>M_{\mathrm{Rep} 2}=M_{\mathrm{Rep} 1}$

$$
\begin{aligned}
\delta b_{i}\left(\mu>M_{\text {Rep } 2}\right) & =\left(\begin{array}{c}
\frac{44}{15} \\
4 \\
\frac{10}{3}
\end{array}\right), \quad \delta m_{i j}\left(\mu>M_{\text {Rep } 2}\right)=\left(\begin{array}{ccc}
\frac{178}{150} & \frac{54}{10} & \frac{80}{15} \\
\frac{18}{10} & 49 & 0 \\
\frac{2}{3} & 0 & \frac{250}{3}
\end{array}\right) \\
\delta \beta_{u}^{(2)}\left(\mu>M_{\text {Rep } 2}\right) & =\frac{200}{9} g_{3}^{4}+\frac{1276}{900} g_{1}^{4}+\frac{6}{2} g_{2}^{4} \\
\delta \beta_{d}^{(2)}\left(\mu>M_{\text {Rep } 2}\right) & =\frac{200}{9} g_{3}^{4}-\frac{44}{900} g_{1}^{4}+\frac{6}{2} g_{2}^{4} \\
\delta \beta_{e}^{(2)}\left(\mu>M_{\text {Rep } 2}\right) & =\frac{44}{100} g_{1}^{4}+\frac{6}{2} g_{2}^{4} \\
\delta \beta_{\lambda}^{(2)}\left(\mu>M_{\text {Rep2 }}\right) & =-\frac{44}{250} g_{1}^{4}\left(12 g_{1}^{2}+20 g_{2}^{2}-25 \lambda\right)-\frac{6}{5} g_{2}^{4}\left(4 g_{1}^{2}+20 g_{2}^{2}-25 \lambda\right)
\end{aligned}
$$

(b) $M_{\text {Rep1 }}>M_{\text {Rep2 }}$

(I) $M_{\mathrm{Rep} 1}>\mu>M_{\mathrm{Rep} 2}$

$\delta b_{i}\left(M_{\text {Rep } 2}<\mu<M_{\text {Rep } 1}\right)=\left(\begin{array}{c}\frac{8}{15} \\ 0 \\ \frac{10}{3}\end{array}\right), \delta m_{i j}\left(M_{\text {Rep } 2}<\mu<M_{\text {Rep } 1}\right)=\left(\begin{array}{ccc}\frac{16}{150} & 0 & \frac{80}{15} \\ 0 & 0 & 0 \\ \frac{2}{3} & 0 & \frac{250}{3}\end{array}\right)$ 


$$
\begin{aligned}
& \delta \beta_{u}^{(2)}\left(M_{\text {Rep } 2}<\mu<M_{\text {Rep } 1}\right)=\frac{200}{9} g_{3}^{4}+\frac{232}{900} g_{1}^{4} \\
& \delta \beta_{d}^{(2)}\left(M_{\text {Rep } 2}<\mu<M_{\text {Rep } 1}\right)=\frac{200}{9} g_{3}^{4}-\frac{8}{900} g_{1}^{4} \\
& \delta \beta_{e}^{(2)}\left(M_{\text {Rep2 }}<\mu<M_{\text {Rep } 1}\right)=\frac{88}{10} g_{2}^{4} \\
& \delta \beta_{\lambda}^{(2)}\left(M_{\text {Rep2 }}<\mu<M_{\text {Rep } 1}\right)=-\frac{8}{250} g_{1}^{4}\left(12 g_{1}^{2}+20 g_{2}^{2}-25 \lambda\right)
\end{aligned}
$$

(II) $\mu>M_{\mathrm{Rep} 1}$

$$
\begin{aligned}
\delta b_{i}\left(\mu>M_{\text {Rep } 1}\right) & =\left(\begin{array}{c}
\frac{44}{15} \\
4 \\
\frac{10}{3}
\end{array}\right), \quad \delta m_{i j}\left(\mu>M_{\text {Rep } 1}\right)=\left(\begin{array}{ccc}
\frac{178}{150} & \frac{54}{10} & \frac{80}{15} \\
\frac{18}{10} & 49 & 0 \\
\frac{2}{3} & 0 & \frac{250}{3}
\end{array}\right) \\
\delta \beta_{u}^{(2)}\left(\mu>M_{\text {Rep } 1}\right) & =\frac{200}{9} g_{3}^{4}+\frac{1276}{900} g_{1}^{4}+\frac{6}{2} g_{2}^{4} \\
\delta \beta_{d}^{(2)}\left(\mu>M_{\text {Rep } 1}\right) & =\frac{200}{9} g_{3}^{4}-\frac{44}{900} g_{1}^{4}+\frac{6}{2} g_{2}^{4} \\
\delta \beta_{e}^{(2)}\left(\mu>M_{\text {Rep } 1}\right) & =\frac{44}{100} g_{1}^{4}+\frac{6}{2} g_{2}^{4} \\
\delta \beta_{\lambda}^{(2)}\left(\mu>M_{\text {Rep } 1}\right) & =-\frac{44}{250} g_{1}^{4}\left(12 g_{1}^{2}+20 g_{2}^{2}-25 \lambda\right)-\frac{6}{5} g_{2}^{4}\left(4 g_{1}^{2}+20 g_{2}^{2}-25 \lambda\right)
\end{aligned}
$$

\section{(c) $M_{\mathrm{Rep} 1}<M_{\mathrm{Rep} 2}$}

(I) $M_{\mathrm{Rep} 1}<\mu<M_{\mathrm{Rep} 2}$

$$
\delta b_{i}\left(M_{\mathrm{Rep} 1}<\mu<M_{\mathrm{Rep} 2}\right)=\left(\begin{array}{c}
\frac{12}{5} \\
4 \\
0
\end{array}\right), \delta m_{i j}\left(M_{\mathrm{Rep} 1}<\mu<M_{\mathrm{Rep} 2}\right)=\left(\begin{array}{ccc}
\frac{54}{50} & \frac{54}{10} & 0 \\
\frac{18}{10} & 49 & 0 \\
0 & 0 & 0
\end{array}\right)
$$

$\delta \beta_{u}^{(2)}\left(M_{\mathrm{Rep} 1}<\mu<M_{\mathrm{Rep} 2}\right)=\frac{1044}{900} g_{1}^{4}+\frac{6}{2} g_{2}^{4}$

$\delta \beta_{d}^{(2)}\left(M_{\mathrm{Rep} 1}<\mu<M_{\mathrm{Rep} 2}\right)=\frac{36}{900} g_{1}^{4}+\frac{6}{2} g_{2}^{4}$

$\delta \beta_{e}^{(2)}\left(M_{\mathrm{Rep} 1}<\mu<M_{\mathrm{Rep} 2}\right)=\frac{396}{100} g_{1}^{4}+\frac{6}{2} g_{2}^{4}$

$\delta \beta_{\lambda}^{(2)}\left(M_{\mathrm{Rep} 1}<\mu<M_{\mathrm{Rep} 2}\right)=-\frac{36}{250} g_{1}^{4}\left(12 g_{1}^{2}+20 g_{2}^{2}-25 \lambda\right)-\frac{6}{5} g_{2}^{4}\left(4 g_{1}^{2}+20 g_{2}^{2}-25 \lambda\right)$

(II) $\mu>M_{\mathrm{Rep} 2}$

$$
\begin{aligned}
\delta b_{i}\left(\mu>M_{\text {Rep } 2}\right) & =\left(\begin{array}{c}
\frac{44}{15} \\
4 \\
\frac{10}{3}
\end{array}\right), \quad \delta m_{i j}\left(\mu>M_{\text {Rep } 2}\right)=\left(\begin{array}{ccc}
\frac{178}{150} & \frac{54}{10} & \frac{80}{15} \\
\frac{18}{10} & 49 & 0 \\
\frac{2}{3} & 0 & \frac{250}{3}
\end{array}\right) \\
\delta \beta_{u}^{(2)}\left(\mu>M_{\text {Rep2 }}\right) & =\frac{200}{9} g_{3}^{4}+\frac{1276}{900} g_{1}^{4}+\frac{6}{2} g_{2}^{4} \\
\delta \beta_{d}^{(2)}\left(\mu>M_{\text {Rep } 2}\right) & =\frac{200}{9} g_{3}^{4}-\frac{44}{900} g_{1}^{4}+\frac{6}{2} g_{2}^{4} \\
\delta \beta_{e}^{(2)}\left(\mu>M_{\text {Rep2 } 2}\right) & =\frac{44}{100} g_{1}^{4}+\frac{6}{2} g_{2}^{4} \\
\delta \beta_{\lambda}^{(2)}\left(\mu>M_{\text {Rep } 2}\right) & =-\frac{44}{250} g_{1}^{4}\left(12 g_{1}^{2}+20 g_{2}^{2}-25 \lambda\right)-\frac{6}{5} g_{2}^{4}\left(4 g_{1}^{2}+20 g_{2}^{2}-25 \lambda\right)
\end{aligned}
$$




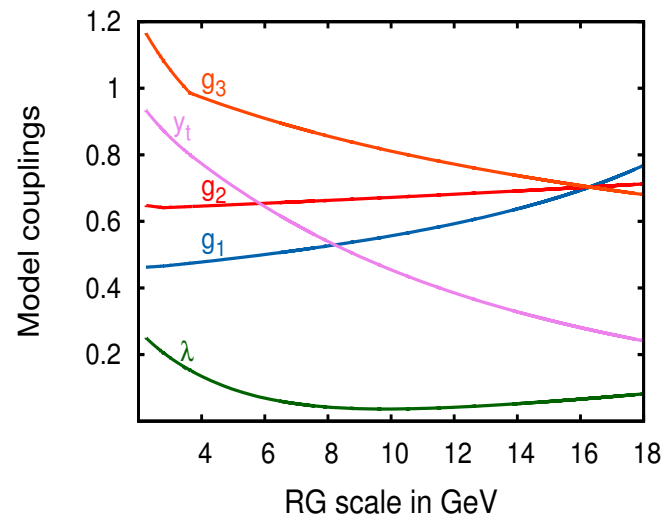

(a)

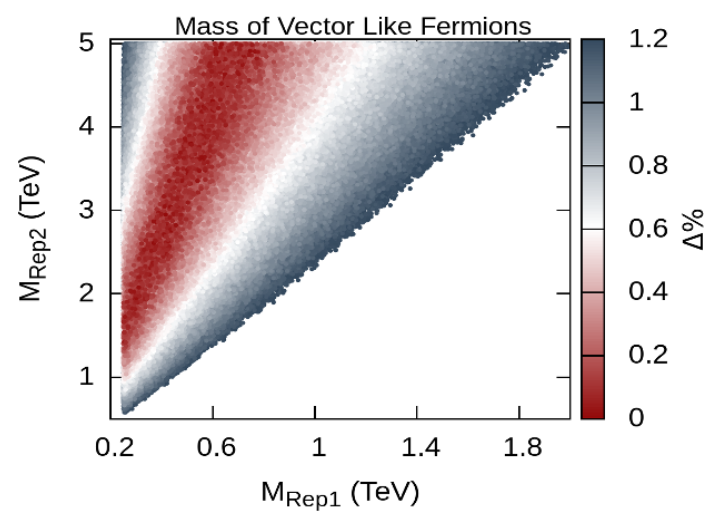

(b)

Figure 3. Model 2: figure (a) Gauge couplings $\left(g_{1}, g_{2}, g_{3}\right)$ unification and Vacuum stability $(\lambda>0)$ plot, considering vector-like fermion in Rep. 1 of mass $620 \mathrm{GeV}$ and Rep. 2 of mass $4310 \mathrm{GeV}$. Figure (b) Mass range allowed for vector-like fermions in Rep.1 and Rep.2 for gauge unification and Vacuum stability.

A sample unification point is shown in figure 2(a), six copies of lepton like vector fermions with degenerate mass of $1210 \mathrm{GeV}$ and one copy of Rep2 with a mass of $1260 \mathrm{GeV}$ is considered. The figure shows unification clearly. The running of $y_{t}$ and $\lambda$ are also shown. The panel figure 2(b) has the mass distribution in Rep1-Rep2 mass plane. The model clearly prefers degeneracy of Rep1 and Rep2 for successful unification.

\subsection{Model 2}

We got six copies of Rep1 $=\left(1,2, \frac{1}{2}\right)$ in mass range between $250 \mathrm{GeV}$ to $2000 \mathrm{GeV}$ and two copies of Rep $2=(8,1,0)$ with mass range from $500 \mathrm{GeV}$ to $5 \mathrm{TeV}$. Similar to the previous model, Rep1 field is lepton like field and thus all the comments are applicable here. Rep2 is gluino like and at the renormalisation level, it can interact with the gluons only and does not have any decay chain. Possibility of any higher dimension decaying operators and its collider phenomenology are studied in the next section 5 .

In the model, $M_{\text {Rep1 }}$ is always less than $M_{\text {Rep2 }}$. The change in the beta functions in the two thresholds are as follows:

$$
\begin{aligned}
& \text { (I) } M_{\text {Rep1 }}<\mu<M_{\text {Rep2 }} \\
& \delta b_{i}\left(M_{\mathrm{Rep} 1}<\mu<M_{\mathrm{Rep} 2}\right)=\left(\begin{array}{c}
\frac{12}{5} \\
4 \\
0
\end{array}\right), \delta m_{i j}\left(M_{\mathrm{Rep} 1}<\mu<M_{\mathrm{Rep} 2}\right)=\left(\begin{array}{ccc}
\frac{54}{50} & \frac{54}{10} & 0 \\
\frac{18}{10} & 49 & 0 \\
0 & 0 & 0
\end{array}\right) \\
& \delta \beta_{u}^{(2)}\left(M_{\mathrm{Rep} 1}<\mu<M_{\mathrm{Rep} 2}\right)=\frac{1044}{900} g_{1}^{4}+\frac{6}{2} g_{2}^{4} \\
& \delta \beta_{d}^{(2)}\left(M_{\text {Rep } 1}<\mu<M_{\text {Rep } 2}\right)=-\frac{36}{900} g_{1}^{4}+\frac{6}{2} g_{2}^{4} \\
& \delta \beta_{e}^{(2)}\left(M_{\mathrm{Rep} 1}<\mu<M_{\mathrm{Rep} 2}\right)=\frac{396}{100} g_{1}^{4}+\frac{6}{2} g_{2}^{4}
\end{aligned}
$$




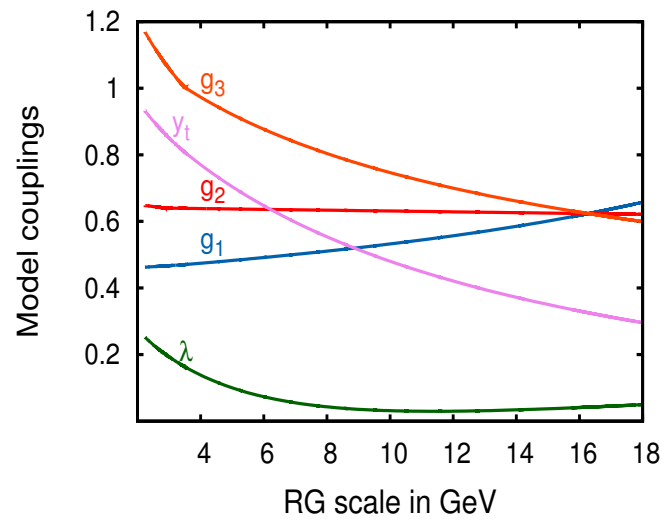

(a)

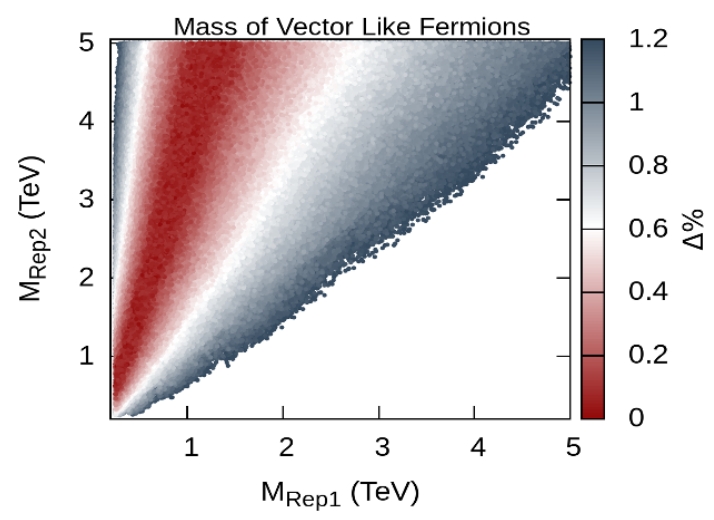

(b)

Figure 4. Model 3: figure (a) Gauge couplings $\left(g_{1}, g_{2}, g_{3}\right)$ unification and Vacuum stability $(\lambda>0)$ plot, considering vector-like fermion in Rep. 1 of mass $800 \mathrm{GeV}$ and Rep. 2 of mass $3030 \mathrm{GeV}$. Figure (b) Mass range allowed for vector-like fermions in Rep.1 and Rep.2 for gauge unification and vacuum stability.

$$
\delta \beta_{\lambda}^{(2)}\left(M_{\mathrm{Rep} 1}<\mu<M_{\mathrm{Rep} 2}\right)=-\frac{36}{250} g_{1}^{4}\left(12 g_{1}^{2}+20 g_{2}^{2}-25 \lambda\right)-\frac{6}{5} g_{2}^{4}\left(4 g_{1}^{2}+20 g_{2}^{2}-25 \lambda\right)
$$

(II) $\mu>M_{\text {Rep2 }}$

$$
\begin{aligned}
\delta b_{i}\left(\mu>M_{\text {Rep } 2}\right) & =\left(\begin{array}{c}
\frac{12}{5} \\
4 \\
4
\end{array}\right), \quad \delta m_{i j}\left(\mu>M_{\text {Rep } 2}\right)=\left(\begin{array}{ccc}
\frac{54}{50} & \frac{54}{10} & 0 \\
\frac{18}{10} & 49 & 0 \\
0 & 0 & 96
\end{array}\right) \\
\delta \beta_{u}^{(2)}\left(\mu>M_{\text {Rep } 2}\right) & =\frac{240}{9} g_{3}^{4}+\frac{1044}{900} g_{1}^{4}+\frac{6}{2} g_{2}^{4} \\
\delta \beta_{d}^{(2)}\left(\mu>M_{\text {Rep } 2}\right) & =\frac{240}{9} g_{3}^{4}-\frac{36}{900} g_{1}^{4}+\frac{6}{2} g_{2}^{4} \\
\delta \beta_{e}^{(2)}\left(\mu>M_{\text {Rep } 2}\right) & =\frac{396}{100} g_{1}^{4}+\frac{6}{2} g_{2}^{4} \\
\delta \beta_{\lambda}^{(2)}\left(\mu>M_{\text {Rep2 }}\right) & =-\frac{36}{250} g_{1}^{4}\left(12 g_{1}^{2}+20 g_{2}^{2}-25 \lambda\right)-\frac{6}{5} g_{2}^{4}\left(4 g_{1}^{2}+20 g_{2}^{2}-25 \lambda\right)
\end{aligned}
$$

A sample unification point is shown in figure 3(a), six copies of lepton like vector fermions with degenerate mass of $620 \mathrm{GeV}$ and two copy of Rep2 with a mass of $4310 \mathrm{GeV}$ is considered. The figure shows unification of gauge couplings as well as running of $y_{t}$ and $\lambda$. Mass distribution in Rep1-Rep2 mass plane is shown in figure 3(b).

\subsection{Model 3}

In this model, we got two copies of Rep1 $=(1,3,0)$ and four copies of Rep2 $=\left(3,1, \frac{1}{3}\right)$. The mass ranges of Rep1 and Rep2 are $(250 \mathrm{GeV}, 5 \mathrm{TeV})$ and $(250 \mathrm{GeV}, 5 \mathrm{TeV})$ respectively. Rep1 can be a viable candidate of type III $[67,68]$ seesaw model with fermion mass of $\mathrm{M}$. The neutrino masses are generically given by a factor $v^{2} / M$, where $v$ is the vacuum expectation value of the Higgs field. For large M (of the order of $10^{14} \mathrm{GeV}$ ), small neutrino 
masses are generated even for Yukawa couplings of $\sim 1$. On the other hand, either smaller Yukawa couplings $\sim 10^{-11}$ (which would not effect the RG running) or extended seesaw mechanisms, such as those of the inverse seesaw models [69], are required to obtain small neutrino masses while keeping $M$ close to a few hundreds of $\mathrm{GeV}$. However, we can also impose appropriate $Z_{N}$ symmetries. This symmetry removes all the Yukawa terms involving these fields at the renormalisable level and only gauge couplings are allowed. ${ }^{6}$ Neutral component of these fermions is a viable dark matter candidate. This type of dark matter are referred as wino like dark matter and have been discussed in [70-73].

Rep2 has same representation like the down quark. This colour vector-like fermion can form a bound state and annihilate to diphoton, dijet etc. event, which we studied in section 5 .

For most points in this model vector-like fermions in Rep1 can be degenerate with vector-like fermions in Rep2 $\left(M_{\operatorname{Rep} 1} \sim M_{\text {Rep2 }}\right)$ as shown in figure 2(b). However, there could be points in which either of the $M_{\mathrm{Rep} 1}=M_{\mathrm{Rep} 2}, M_{\mathrm{Rep} 1}>M_{\mathrm{Rep} 2}$ and $M_{\mathrm{Rep} 1}<M_{\mathrm{Rep} 2}$ are possible. The change in the beta functions in these possibilities are as follows:

$$
\begin{aligned}
& \text { (a) } M_{\text {Rep } 1}=M_{\text {Rep2 }} \\
& \text { (I) } \mu>M_{\text {Rep } 2}=M_{\text {Rep } 1} \\
& \delta b_{i}\left(\mu>M_{\text {Rep2 }}\right)=\left(\begin{array}{l}
\frac{16}{15} \\
\frac{16}{6} \\
\frac{16}{6}
\end{array}\right), \quad \delta m_{i j}\left(\mu>M_{\text {Rep2 }}\right)=\left(\begin{array}{ccc}
\frac{16}{75} & 0 & \frac{64}{15} \\
0 & \frac{128}{3} & 0 \\
\frac{16}{30} & 0 & \frac{152}{3}
\end{array}\right) \\
& \delta \beta_{u}^{(2)}\left(\mu>M_{\text {Rep } 2}\right)=\frac{160}{9} g_{3}^{4}+\frac{464}{900} g_{1}^{4}+\frac{4}{2} g_{2}^{4} \\
& \delta \beta_{d}^{(2)}\left(\mu>M_{\text {Rep2 }}\right)=\frac{160}{9} g_{3}^{4}-\frac{16}{900} g_{1}^{4}+\frac{4}{2} g_{2}^{4} \\
& \delta \beta_{e}^{(2)}\left(\mu>M_{\text {Rep2 }}\right)=\frac{176}{100} g_{1}^{4}+\frac{4}{2} g_{2}^{4} \\
& \delta \beta_{\lambda}^{(2)}\left(\mu>M_{\text {Rep2 }}\right)=-\frac{16}{250} g_{1}^{4}\left(12 g_{1}^{2}+20 g_{2}^{2}-25 \lambda\right)-\frac{4}{5} g_{2}^{4}\left(4 g_{1}^{2}+20 g_{2}^{2}-25 \lambda\right)
\end{aligned}
$$

(b) $M_{\text {Rep1 }}<M_{\text {Rep2 }}$

(I) $M_{\mathrm{Rep} 1}<\mu<M_{\mathrm{Rep} 2}$

$$
\begin{aligned}
\delta b_{i}\left(M_{\text {Rep } 1}<\mu<M_{\text {Rep } 2}\right) & =\left(\begin{array}{c}
0 \\
\frac{16}{6} \\
0
\end{array}\right), \delta m_{i j}\left(M_{\text {Rep } 1}<\mu<M_{\text {Rep } 2}\right)=\left(\begin{array}{ccc}
0 & 0 & 0 \\
0 & \frac{128}{3} & 0 \\
0 & 0 & 0
\end{array}\right) \\
\delta \beta_{u}^{(2)}\left(M_{\text {Rep } 1}<\mu<M_{\text {Rep } 2}\right) & =\frac{4}{2} g_{2}^{4} \\
\delta \beta_{d}^{(2)}\left(M_{\text {Rep } 1}<\mu<M_{\text {Rep } 2}\right) & =\frac{4}{2} g_{2}^{4} \\
\delta \beta_{e}^{(2)}\left(M_{\text {Rep } 1}<\mu<M_{\text {Rep } 2}\right) & =\frac{4}{2} g_{2}^{4}
\end{aligned}
$$

\footnotetext{
${ }^{6}$ Seesaw requires Yukawa couplings, our model does not have a seesaw mechanism for neutrino masses.
} 


$$
\delta \beta_{\lambda}^{(2)}\left(M_{\mathrm{Rep} 1}<\mu<M_{\mathrm{Rep} 2}\right)=-\frac{4}{5} g_{2}^{4}\left(4 g_{1}^{2}+20 g_{2}^{2}-25 \lambda\right)
$$

(II) $\mu>M_{\text {Rep2 }}$

$$
\begin{aligned}
\delta b_{i}\left(\mu>M_{\text {Rep } 2}\right) & =\left(\begin{array}{c}
\frac{16}{15} \\
\frac{16}{6} \\
\frac{16}{6}
\end{array}\right), \quad \delta m_{i j}\left(\mu>M_{\text {Rep } 2}\right)=\left(\begin{array}{ccc}
\frac{16}{75} & 0 & \frac{64}{15} \\
0 & \frac{128}{3} & 0 \\
\frac{16}{30} & 0 & \frac{152}{3}
\end{array}\right) \\
\delta \beta_{u}^{(2)}\left(\mu>M_{\text {Rep } 2}\right) & =\frac{160}{9} g_{3}^{4}+\frac{464}{900} g_{1}^{4}+\frac{4}{2} g_{2}^{4} \\
\delta \beta_{d}^{(2)}\left(\mu>M_{\text {Rep } 2}\right) & =\frac{160}{9} g_{3}^{4}-\frac{16}{900} g_{1}^{4}+\frac{4}{2} g_{2}^{4} \\
\delta \beta_{e}^{(2)}\left(\mu>M_{\text {Rep2 } 2}\right) & =\frac{176}{100} g_{1}^{4}+\frac{4}{2} g_{2}^{4} \\
\delta \beta_{\lambda}^{(2)}\left(\mu>M_{\text {Rep2 }}\right) & =-\frac{16}{250} g_{1}^{4}\left(12 g_{1}^{2}+20 g_{2}^{2}-25 \lambda\right)-\frac{4}{5} g_{2}^{4}\left(4 g_{1}^{2}+20 g_{2}^{2}-25 \lambda\right)
\end{aligned}
$$

(c) $M_{\mathrm{Rep} 2}<M_{\mathrm{Rep} 1}$

(I) $M_{\mathrm{Rep} 2}<\mu<M_{\operatorname{Rep} 1}$

$$
\delta b_{i}\left(M_{\text {Rep } 2}<\mu<M_{\text {Rep } 1}\right)=\left(\begin{array}{c}
\frac{16}{15} \\
0 \\
\frac{16}{6}
\end{array}\right), \quad \delta m_{i j}\left(M_{\text {Rep } 2}<\mu<M_{\text {Rep } 1}\right)=\left(\begin{array}{ccc}
\frac{16}{75} & 0 & \frac{64}{15} \\
0 & 0 & 0 \\
\frac{16}{30} & 0 & \frac{152}{3}
\end{array}\right)
$$

$$
\begin{aligned}
& \delta \beta_{u}^{(2)}\left(M_{\mathrm{Rep} 2}<\mu<M_{\mathrm{Rep} 1}\right)=\frac{160}{9} g_{3}^{4}+\frac{464}{900} g_{1}^{4} \\
& \delta \beta_{d}^{(2)}\left(M_{\mathrm{Rep} 2}<\mu<M_{\mathrm{Rep} 1}\right)=\frac{160}{9} g_{3}^{4}-\frac{16}{900} g_{1}^{4} \\
& \delta \beta_{e}^{(2)}\left(M_{\mathrm{Rep} 2}<\mu<M_{\mathrm{Rep} 1}\right)=\frac{176}{100} g_{1}^{4} \\
& \delta \beta_{\lambda}^{(2)}\left(M_{\mathrm{Rep} 2}<\mu<M_{\mathrm{Rep} 1}\right)=-\frac{16}{250} g_{1}^{4}\left(12 g_{1}^{2}+20 g_{2}^{2}-25 \lambda\right)
\end{aligned}
$$

(II) $\mu>M_{\text {Rep1 }}$

$$
\begin{aligned}
\delta b_{i}\left(\mu>M_{\text {Rep } 1}\right) & =\left(\begin{array}{c}
\frac{16}{15} \\
\frac{16}{6} \\
\frac{16}{6}
\end{array}\right), \quad \delta m_{i j}\left(\mu>M_{\text {Rep } 1}\right)=\left(\begin{array}{ccc}
\frac{16}{75} & 0 & \frac{64}{15} \\
0 & \frac{128}{3} & 0 \\
\frac{16}{30} & 0 & \frac{152}{3}
\end{array}\right) \\
\delta \beta_{u}^{(2)}\left(\mu>M_{\text {Rep } 1}\right) & =\frac{160}{9} g_{3}^{4}+\frac{464}{900} g_{1}^{4}+\frac{4}{2} g_{2}^{4} \\
\delta \beta_{d}^{(2)}\left(\mu>M_{\text {Rep } 1}\right) & =\frac{160}{9} g_{3}^{4}-\frac{16}{900} g_{1}^{4}+\frac{4}{2} g_{2}^{4} \\
\delta \beta_{e}^{(2)}\left(\mu>M_{\text {Rep } 1}\right) & =\frac{176}{100} g_{1}^{4}+\frac{4}{2} g_{2}^{4} \\
\delta \beta_{\lambda}^{(2)}\left(\mu>M_{\text {Rep } 1}\right) & =-\frac{16}{250} g_{1}^{4}\left(12 g_{1}^{2}+20 g_{2}^{2}-25 \lambda\right)-\frac{4}{5} g_{2}^{4}\left(4 g_{1}^{2}+20 g_{2}^{2}-25 \lambda\right)
\end{aligned}
$$

In figure 4(a), a sample of gauge couplings, $y_{t}$ and $\lambda$ running is shown with two copies of weak-isospin triplet vector-like fermions with degenerate mass of $800 \mathrm{GeV}$ and four copies of 


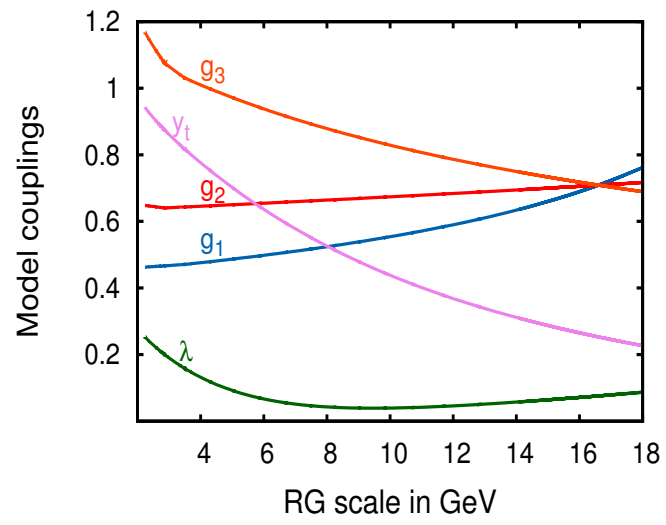

(a)

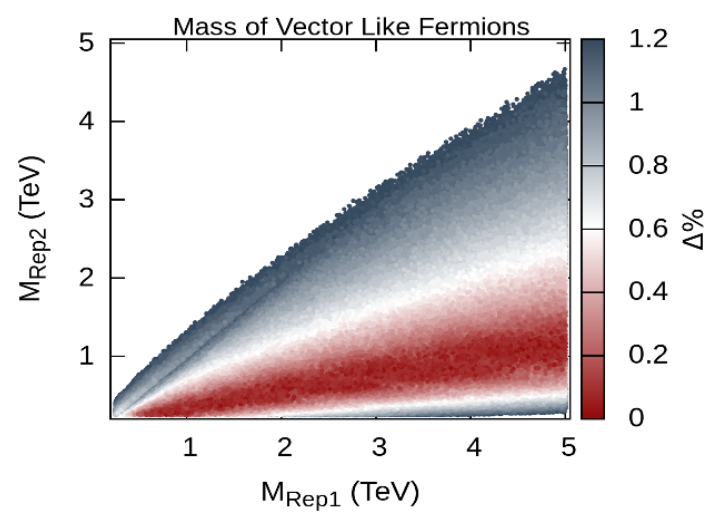

(b)

Figure 5. Model 4: figure (a) Gauge couplings $\left(g_{1}, g_{2}, g_{3}\right)$ unification and Vacuum stability $(\lambda>0)$ plot, considering vector-like fermion in Rep. 1 of mass $3175 \mathrm{GeV}$ and Rep. 2 of mass $730 \mathrm{GeV}$. Figure (b) Mass range allowed for vector-like fermions in Rep.1 and Rep.2 for gauge unification and vacuum stability.

bottom like vector quark with a mass of $3030 \mathrm{GeV}$. Figure 4(b) shows the mass distribution in Rep1-Rep2 mass plane.

\subsection{Model 4}

This model is interesting as representations of the vector-like matter are like up quarks (Rep1) and left handed quark (Rep2). They appear in two copies for each and their mass ranges are $(500 \mathrm{GeV}, 5 \mathrm{TeV})$ and $(250 \mathrm{GeV}, 4.5 \mathrm{TeV})$ respectively. These vector-like quark can be probed at LHC as a bound state, which is studied in section 5 .

In the model, $M_{\text {Rep1 }}$ is greater than $M_{\text {Rep2 }}$. However, some parameters of vector like fermion mass have $M_{\mathrm{Rep} 1}<M_{\mathrm{Rep} 2}$ and $M_{\mathrm{Rep} 1}=M_{\mathrm{Rep} 2}$. The change in the beta functions in these three possibilities are as follows:

(a) $M_{\text {Rep1 }}=M_{\text {Rep2 }}$

(I) $\mu>M_{\operatorname{Rep} 2}=M_{\operatorname{Rep} 1}$

$$
\begin{aligned}
\delta b_{i}\left(\mu>M_{\text {Rep } 1}\right) & =\left(\begin{array}{c}
\frac{12}{5} \\
4 \\
4
\end{array}\right), \quad \delta m_{i j}\left(\mu>M_{\text {Rep } 1}\right)=\left(\begin{array}{ccc}
\frac{258}{150} & \frac{3}{5} & \frac{144}{15} \\
\frac{1}{5} & 49 & 16 \\
\frac{18}{15} & 6 & 76
\end{array}\right) \\
\delta \beta_{u}^{(2)}\left(\mu>M_{\text {Rep } 1}\right) & =\frac{240}{9} g_{3}^{4}+\frac{1044}{900} g_{1}^{4}+\frac{6}{2} g_{2}^{4} \\
\delta \beta_{d}^{(2)}\left(\mu>M_{\text {Rep } 1}\right) & =\frac{240}{9} g_{3}^{4}-\frac{36}{900} g_{1}^{4}+\frac{6}{2} g_{2}^{4} \\
\delta \beta_{e}^{(2)}\left(\mu>M_{\text {Rep } 1}\right) & =\frac{396}{100} g_{1}^{4}+\frac{6}{2} g_{2}^{4} \\
\delta \beta_{\lambda}^{(2)}\left(\mu>M_{\text {Rep } 1}\right) & =-\frac{36}{250} g_{1}^{4}\left(12 g_{1}^{2}+20 g_{2}^{2}-25 \lambda\right)-\frac{6}{5} g_{2}^{4}\left(4 g_{1}^{2}+20 g_{2}^{2}-25 \lambda\right)
\end{aligned}
$$


(b) $M_{\text {Rep2 }}<M_{\text {Rep1 }}$

(I) $M_{\mathrm{Rep} 2}<\mu<M_{\mathrm{Rep} 1}$

$\delta b_{i}\left(M_{\text {Rep } 2}<\mu<M_{\text {Rep1 }}\right)=\left(\begin{array}{c}\frac{4}{15} \\ 4 \\ \frac{8}{3}\end{array}\right), \delta m_{i j}\left(M_{\text {Rep2 }}<\mu<M_{\text {Rep } 1}\right)=\left(\begin{array}{ccc}\frac{2}{150} & \frac{3}{5} & \frac{16}{15} \\ \frac{1}{5} & 49 & 16 \\ \frac{2}{15} & 6 & \frac{152}{3}\end{array}\right)$

$\delta \beta_{u}^{(2)}\left(M_{\mathrm{Rep} 2}<\mu<M_{\mathrm{Rep} 1}\right)=\frac{160}{9} g_{3}^{4}+\frac{116}{900} g_{1}^{4}+\frac{6}{2} g_{2}^{4}$

$\delta \beta_{d}^{(2)}\left(M_{\mathrm{Rep} 2}<\mu<M_{\mathrm{Rep} 1}\right)=\frac{160}{9} g_{3}^{4}(4)-\frac{4}{900} g_{1}^{4}+\frac{6}{2} g_{2}^{4}$

$\delta \beta_{e}^{(2)}\left(M_{\mathrm{Rep} 2}<\mu<M_{\mathrm{Rep} 1}\right)=\frac{44}{100} g_{1}^{4}+\frac{6}{2} g_{2}^{4}$

$\delta \beta_{\lambda}^{(2)}\left(M_{\text {Rep } 2}<\mu<M_{\text {Rep } 1}\right)=-\frac{4}{250} g_{1}^{4}\left(12 g_{1}^{2}+20 g_{2}^{2}-25 \lambda\right)-\frac{6}{5} g_{2}^{4}\left(4 g_{1}^{2}+20 g_{2}^{2}-25 \lambda\right)$

(II) $\mu>M_{\operatorname{Rep} 1}$

$$
\begin{aligned}
\delta b_{i}\left(\mu>M_{\text {Rep } 1}\right) & =\left(\begin{array}{c}
\frac{12}{5} \\
4 \\
4
\end{array}\right), \quad \delta m_{i j}\left(\mu>M_{\text {Rep } 1}\right)=\left(\begin{array}{ccc}
\frac{258}{150} & \frac{3}{5} & \frac{144}{15} \\
\frac{1}{5} & 49 & 16 \\
\frac{18}{15} & 6 & 76
\end{array}\right) \\
\delta \beta_{u}^{(2)}\left(\mu>M_{\text {Rep } 1}\right) & =\frac{240}{9} g_{3}^{4}+\frac{1044}{900} g_{1}^{4}+\frac{6}{2} g_{2}^{4} \\
\delta \beta_{d}^{(2)}\left(\mu>M_{\text {Rep } 1}\right) & =\frac{240}{9} g_{3}^{4}-\frac{36}{900} g_{1}^{4}+\frac{6}{2} g_{2}^{4} \\
\delta \beta_{e}^{(2)}\left(\mu>M_{\text {Rep } 1}\right) & =\frac{396}{100} g_{1}^{4}+\frac{6}{2} g_{2}^{4} \\
\delta \beta_{\lambda}^{(2)}\left(\mu>M_{\text {Rep } 1}\right) & =-\frac{36}{250} g_{1}^{4}\left(12 g_{1}^{2}+20 g_{2}^{2}-25 \lambda\right)-\frac{6}{5} g_{2}^{4}\left(4 g_{1}^{2}+20 g_{2}^{2}-25 \lambda\right)
\end{aligned}
$$

\section{(c) $M_{\mathrm{Rep} 1}<M_{\mathrm{Rep} 2}$}

(I) $M_{\mathrm{Rep} 1}<\mu<M_{\operatorname{Rep} 2}$

$$
\begin{aligned}
\delta b_{i}\left(M_{\text {Rep } 1}<\mu<M_{\text {Rep } 2}\right) & =\left(\begin{array}{c}
\frac{32}{15} \\
0 \\
\frac{4}{3}
\end{array}\right), \delta m_{i j}\left(M_{\text {Rep } 1}<\mu<M_{\text {Rep } 2}\right)=\left(\begin{array}{ccc}
\frac{256}{150} & 0 & \frac{128}{15} \\
0 & 49 & 0 \\
\frac{16}{15} & 0 & \frac{76}{3}
\end{array}\right) \\
\delta \beta_{u}^{(2)}\left(M_{\text {Rep } 1}<\mu<M_{\text {Rep } 2}\right) & =\frac{80}{9} g_{3}^{4}+\frac{928}{900} g_{1}^{4} \\
\delta \beta_{d}^{(2)}\left(M_{\text {Rep } 1}<\mu<M_{\text {Rep } 2}\right) & =\frac{80}{9} g_{3}^{4}(4)-\frac{32}{900} g_{1}^{4} \\
\delta \beta_{e}^{(2)}\left(M_{\text {Rep } 1}<\mu<M_{\text {Rep } 2}\right) & =\frac{352}{100} g_{1}^{4} \\
\delta \beta_{\lambda}^{(2)}\left(M_{\text {Rep } 1}<\mu<M_{\text {Rep } 2}\right) & =-\frac{32}{250} g_{1}^{4}\left(12 g_{1}^{2}+20 g_{2}^{2}-25 \lambda\right)
\end{aligned}
$$




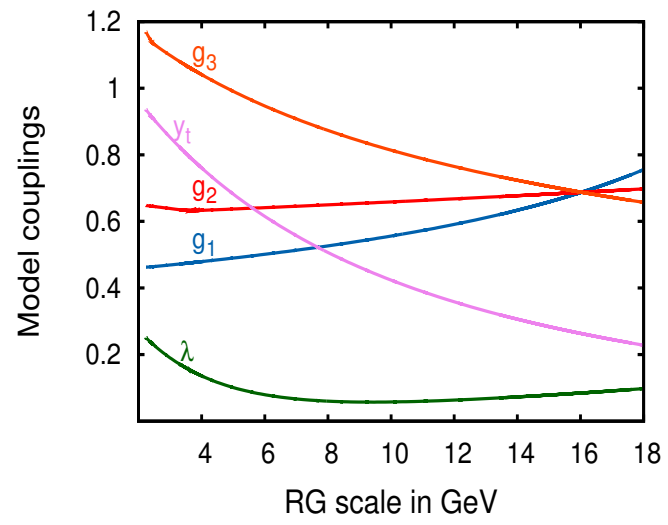

(a)

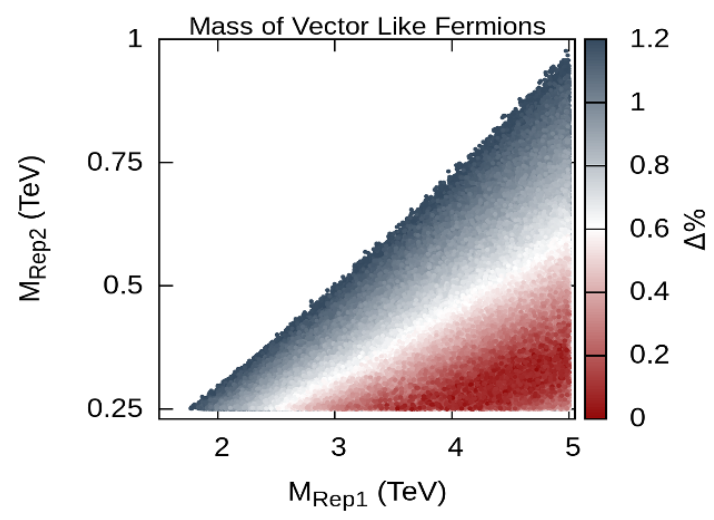

(b)

Figure 6. Model 5: figure (a) Gauge couplings $\left(g_{1}, g_{2}, g_{3}\right)$ unification and Vacuum stability $(\lambda>0)$ plot, considering vector-like fermion in Rep. 1 of mass $4.16 \mathrm{TeV}$ and Rep. 2 of mass $280 \mathrm{GeV}$. Figure (b) Mass range allowed for vector-like fermions in Rep.1 and Rep.2 for gauge unification and vacuum stability.

$$
\begin{aligned}
\text { (II) } \mu>M_{\text {Rep2 }} & \\
\delta b_{i}\left(\mu>M_{\text {Rep } 2}\right) & =\left(\begin{array}{c}
\frac{12}{5} \\
4 \\
4
\end{array}\right), \quad \delta m_{i j}\left(\mu>M_{\text {Rep } 2}\right)=\left(\begin{array}{ccc}
\frac{258}{150} & \frac{3}{5} & \frac{144}{15} \\
\frac{1}{5} & 49 & 16 \\
\frac{18}{15} & 6 & 76
\end{array}\right) \\
\delta \beta_{u}^{(2)}\left(\mu>M_{\text {Rep } 2}\right) & =\frac{240}{9} g_{3}^{4}+\frac{1044}{900} g_{1}^{4}+\frac{6}{2} g_{2}^{4} \\
\delta \beta_{d}^{(2)}\left(\mu>M_{\text {Rep } 2}\right) & =\frac{240}{9} g_{3}^{4}-\frac{36}{900} g_{1}^{4}+\frac{6}{2} g_{2}^{4} \\
\delta \beta_{e}^{(2)}\left(\mu>M_{\text {Rep2 }}\right) & =\frac{396}{100} g_{1}^{4}+\frac{6}{2} g_{2}^{4} \\
\delta \beta_{\lambda}^{(2)}\left(\mu>M_{\text {Rep2 }}\right) & =-\frac{36}{250} g_{1}^{4}\left(12 g_{1}^{2}+20 g_{2}^{2}-25 \lambda\right)-\frac{6}{5} g_{2}^{4}\left(4 g_{1}^{2}+20 g_{2}^{2}-25 \lambda\right)
\end{aligned}
$$

The running of gauge couplings, $y_{t}$ and $\lambda$ are shown in figure $5(\mathrm{a})$, considering two copies of top like vector fermions with degenerate mass of $3175 \mathrm{GeV}$ and two copies of left handed vector-like quark with a mass of $730 \mathrm{GeV}$. Figure 5(b) shows the mass distribution in Rep1-Rep2 mass plane.

\subsection{Model 5}

This Model consist of 3 copies of vector-like fermion $(1,3,0)$, which is triplet under $\mathrm{SU}(2)$ representation (Rep1) and one copy of vector-like fermion $\left(6,1, \frac{2}{3}\right)$ which is sextet under $\mathrm{SU}(3)$ representation (Rep2). The mass range of Rep1 and Rep2 are (1.8 TeV to $5 \mathrm{TeV})$ and $(250 \mathrm{GeV}$ to $950 \mathrm{GeV})$ respectively. The possible scenarios of Rep1 has been discussed in Model 3 and Rep2 has been mentioned in Model 1 with hypercharge 2/3. In the model, $M_{\mathrm{Rep} 1}$ is greater than $M_{\mathrm{Rep} 2}$. 
The change in the beta functions in the two thresholds are as follows:

(I) $M_{\text {Rep2 }}<\mu<M_{\text {Rep1 }}$

$$
\begin{aligned}
\delta b_{i}\left(M_{\text {Rep } 2}<\mu<M_{\text {Rep } 1}\right) & =\left(\begin{array}{c}
\frac{96}{45} \\
0 \\
\frac{10}{3}
\end{array}\right), \delta m_{i j}\left(M_{\text {Rep } 2}<\mu<M_{\text {Rep } 1}\right)=\left(\begin{array}{ccc}
\frac{128}{75} & 0 & \frac{64}{3} \\
0 & 0 & 0 \\
\frac{8}{3} & 0 & \frac{250}{3}
\end{array}\right) \\
\delta \beta_{u}^{(2)}\left(M_{\text {Rep2 }}<\mu<M_{\text {Rep } 1}\right) & =\frac{200}{9} g_{3}^{4}+\frac{232}{225} g_{1}^{4} \\
\delta \beta_{d}^{(2)}\left(M_{\text {Rep2 }}<\mu<M_{\text {Rep } 1}\right) & =\frac{40}{9} g_{3}^{4}(5)-\frac{32}{900} g_{1}^{4} \\
\delta \beta_{e}^{(2)}\left(M_{\text {Rep2 }}<\mu<M_{\text {Rep } 1}\right) & =\frac{352}{100} g_{1}^{4} \\
\delta \beta_{\lambda}^{(2)}\left(M_{\text {Rep2 }}<\mu<M_{\text {Rep } 1}\right) & =-\frac{32}{250} g_{1}^{4}\left(12 g_{1}^{2}+20 g_{2}^{2}-25 \lambda\right)
\end{aligned}
$$

(II) $\mu>M_{\text {Rep1 }}$

$$
\begin{aligned}
\delta b_{i}\left(\mu>M_{\text {Rep } 1}\right) & =\left(\begin{array}{c}
\frac{96}{45} \\
4 \\
\frac{10}{3}
\end{array}\right), \quad \delta m_{i j}\left(\mu>M_{\text {Rep } 1}\right)=\left(\begin{array}{ccc}
\frac{128}{75} & 0 & \frac{64}{3} \\
0 & 64 & 0 \\
\frac{8}{3} & 0 & \frac{250}{3}
\end{array}\right) \\
\delta \beta_{u}^{(2)}\left(\mu>M_{\text {Rep } 1}\right) & =\frac{200}{9} g_{3}^{4}+\frac{232}{225} g_{1}^{4}+\frac{6}{2} g_{2}^{4} \\
\delta \beta_{d}^{(2)}\left(\mu>M_{\text {Rep } 1}\right) & =\frac{200}{9} g_{3}^{4}-\frac{32}{900} g_{1}^{4}+\frac{6}{2} g_{2}^{4} \\
\delta \beta_{e}^{(2)}\left(\mu>M_{\text {Rep } 1}\right) & =\frac{352}{100} g_{1}^{4}+\frac{6}{2} g_{2}^{4} \\
\delta \beta_{\lambda}^{(2)}\left(\mu>M_{\text {Rep } 1}\right) & =-\frac{32}{250} g_{1}^{4}\left(12 g_{1}^{2}+20 g_{2}^{2}-25 \lambda\right)-\frac{6}{5} g_{2}^{4}\left(4 g_{1}^{2}+20 g_{2}^{2}-25 \lambda\right)
\end{aligned}
$$

A sample unification point is shown in figure 6(a), three copies of weak isospin triplet vector-like fermions with degenerate mass of $4.16 \mathrm{TeV}$ and one copy of color sextet vector-like fermion with a mass of $280 \mathrm{GeV}$ is considered. The figure 6(a) shows unification clearly. Figure 6(b) shows the mass distribution in Rep1-Rep2 mass plane.

\subsection{Model 6}

This model consist of one copy of $\operatorname{Rep} 1=\left(1,4, \frac{1}{2}\right)$ and two copies of $\operatorname{Rep} 2=\left(6,1, \frac{2}{3}\right)$. The mass range for Rep1 and Rep2 are $(250 \mathrm{GeV}$ to $2 \mathrm{TeV})$ and $(1 \mathrm{TeV}$ to $5 \mathrm{TeV})$ respectively. The Rep1 is fourplet under $\mathrm{SU}(2)$ representation and has been studied under minimal dark matter in ref. [70]. To our Knowlegde this is a first time it appeared in the unification of gauge coupling. Rep2 is exotic sextet under SU(3), which we discussed in Model 5 . In the model, $M_{\text {Rep2 }}$ is greater than $M_{\text {Rep1 }}$. The change in the beta functions in the two thresholds are as follows: 


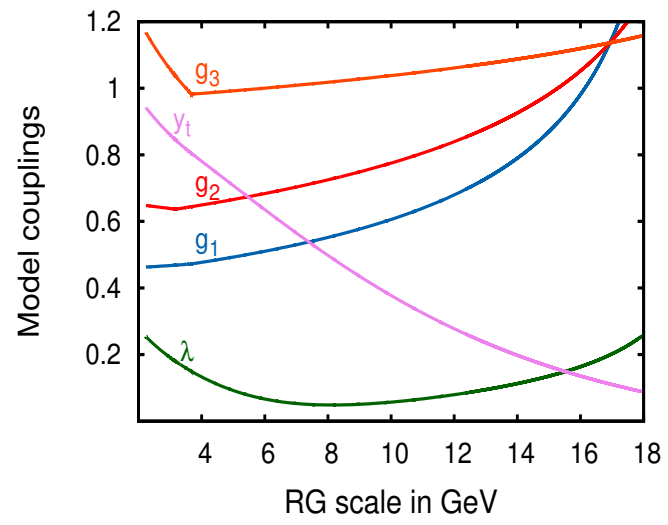

(a)

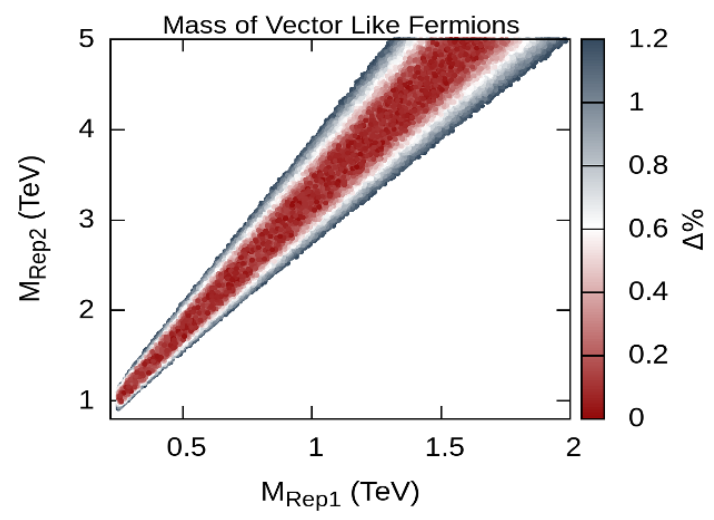

(b)

Figure 7. Model 6: figure (a) Gauge couplings $\left(g_{1}, g_{2}, g_{3}\right)$ unification and Vacuum stability $(\lambda>0)$ plot, considering vector-like fermion in Rep. 1 of mass $1.51 \mathrm{TeV}$ and Rep.2 of mass $4.81 \mathrm{TeV}$. Figure (b) Mass range allowed for vector-like fermions in Rep.1 and Rep.2 for gauge unification and vacuum stability.

(I) $M_{\text {Rep1 }}<\mu<M_{\text {Rep2 }}$

$$
\begin{aligned}
\delta b_{i}\left(M_{\mathrm{Rep} 1}<\mu<M_{\mathrm{Rep} 2}\right) & =\left(\begin{array}{c}
\frac{8}{10} \\
\frac{20}{3} \\
0
\end{array}\right), \delta m_{i j}\left(M_{\mathrm{Rep} 1}<\mu<M_{\mathrm{Rep} 2}\right)=\left(\begin{array}{ccc}
\frac{9}{25} & 9 & 0 \\
3 & \frac{425}{3} & 0 \\
0 & 0 & 0
\end{array}\right) \\
\delta \beta_{u}^{(2)}\left(M_{\mathrm{Rep} 1}<\mu<M_{\mathrm{Rep} 2}\right) & =\frac{348}{900} g_{1}^{4}+\frac{10}{2} g_{2}^{4} \\
\delta \beta_{d}^{(2)}\left(M_{\mathrm{Rep} 1}<\mu<M_{\mathrm{Rep} 2}\right) & =\frac{12}{900} g_{1}^{4}+\frac{10}{2} g_{2}^{4} \\
\delta \beta_{e}^{(2)}\left(M_{\mathrm{Rep} 1}<\mu<M_{\mathrm{Rep} 2}\right) & =\frac{132}{100} g_{1}^{4}+\frac{10}{2} g_{2}^{4} \\
\delta \beta_{\lambda}^{(2)}\left(M_{\mathrm{Rep} 1}<\mu<M_{\mathrm{Rep} 2}\right) & =-\frac{12}{250} g_{1}^{4}\left(12 g_{1}^{2}+20 g_{2}^{2}-25 \lambda\right)-\frac{10}{5} g_{2}^{4}\left(4 g_{1}^{2}+20 g_{2}^{2}-25 \lambda\right)
\end{aligned}
$$

(II) $\mu>M_{\text {Rep2 }}$

$$
\begin{aligned}
\delta b_{i}\left(\mu>M_{\text {Rep } 2}\right) & =\left(\begin{array}{c}
\frac{76}{15} \\
\frac{20}{3} \\
\frac{20}{3}
\end{array}\right), \quad \delta m_{i j}\left(\mu>M_{\text {Rep } 2}\right)=\left(\begin{array}{ccc}
\frac{283}{75} & 9 & \frac{128}{3} \\
3 & \frac{425}{3} & 0 \\
\frac{16}{3} & 0 & \frac{500}{3}
\end{array}\right) \\
\delta \beta_{u}^{(2)}\left(\mu>M_{\text {Rep } 2}\right) & =\frac{400}{9} g_{3}^{4}+\frac{551}{225} g_{1}^{4}+\frac{10}{2} g_{2}^{4} \\
\delta \beta_{d}^{(2)}\left(\mu>M_{\text {Rep } 2}\right) & =\frac{400}{9} g_{3}^{4}-\frac{76}{900} g_{1}^{4}+\frac{10}{2} g_{2}^{4} \\
\delta \beta_{e}^{(2)}\left(\mu>M_{\text {Rep } 2}\right) & =\frac{836}{100} g_{1}^{4}+\frac{10}{2} g_{2}^{4} \\
\delta \beta_{\lambda}^{(2)}\left(\mu>M_{\text {Rep } 2}\right) & =-\frac{76}{250} g_{1}^{4}\left(12 g_{1}^{2}+20 g_{2}^{2}-25 \lambda\right)-\frac{10}{5} g_{2}^{4}\left(4 g_{1}^{2}+20 g_{2}^{2}-25 \lambda\right)
\end{aligned}
$$




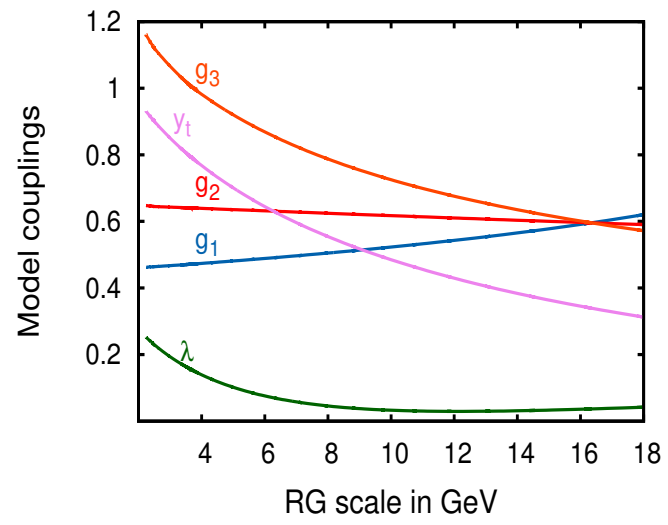

(a)

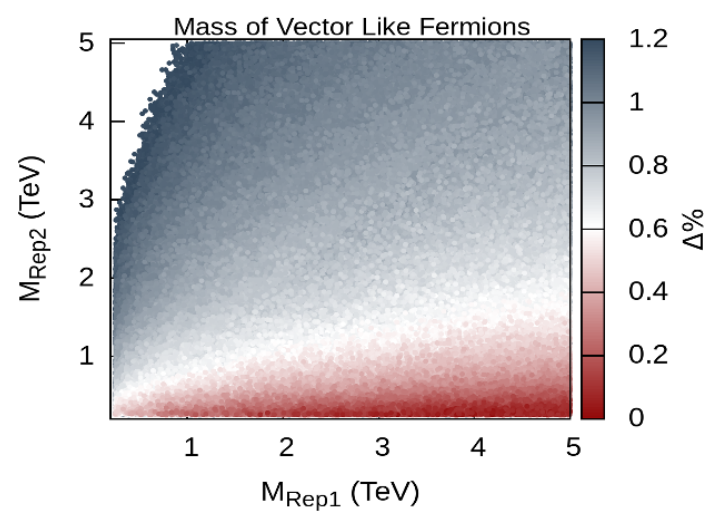

(b)

Figure 8. Model 7: figure (a) Gauge couplings $\left(g_{1}, g_{2}, g_{3}\right)$ unification and Vacuum stability $(\lambda>0)$ plot, considering vector-like fermion in Rep.1 of mass $4.65 \mathrm{TeV}$ and Rep.2 of mass $309 \mathrm{GeV}$. Figure (b) Mass range allowed for vector-like fermions in Rep.1 and Rep.2 for gauge unification and vacuum stability.

Gauge coupling unification and running of $y_{t}$ and $\lambda$ are also shown in figure 7(a), with one copy of weak isospin fourplet vector-like fermions with degenerate mass of $1.51 \mathrm{TeV}$ and two copies of color sextet vector-like fermion with a mass of $4.81 \mathrm{TeV}$. The figure 7 (b) has the mass distribution in Rep1-Rep2 mass plane.

\section{7 $\quad$ Model 7}

This model consist of one copy of $\operatorname{Rep} 1=\left(3,1, \frac{1}{3}\right)$ and two copies of $\operatorname{Rep} 2=\left(3,2, \frac{1}{6}\right)$. The mass range for Rep1 and Rep2 are $(250 \mathrm{GeV}$ to $5 \mathrm{TeV})$ and $(250 \mathrm{GeV}$ to $5 \mathrm{TeV})$ respectively. Representation one has been discussed in Model 4 with Rep1 having hypercharge $2 / 3$. The difference can been studied with their bound state decay to diphoton channel, as shown in section 5. In this model, there could be points in which either of the $M_{\text {Rep1 }} \sim M_{\text {Rep2 }}$, $M_{\text {Rep1 }}>M_{\text {Rep2 }}$ and $M_{\text {Rep1 }}<M_{\text {Rep2 }}$ are possible. The change in the beta functions in the three conditions are as follows:

$$
\begin{aligned}
& \text { (a) } M_{\mathrm{Rep} 1}=M_{\mathrm{Rep} 2} \\
& \text { (I) } \mu>M_{\operatorname{Rep} 2}=M_{\mathrm{Rep} 1} \\
& \delta b_{i}\left(\mu>M_{\text {Rep } 1}\right)=\left(\begin{array}{c}
\frac{4}{10} \\
2 \\
2
\end{array}\right), \quad \delta m_{i j}\left(\mu>M_{\text {Rep } 1}\right)=\left(\begin{array}{ccc}
\frac{6}{100} & \frac{3}{10} & \frac{16}{10} \\
\frac{1}{10} & \frac{245}{10} & 8 \\
\frac{2}{10} & 3 & 38
\end{array}\right) \\
& \delta \beta_{u}^{(2)}\left(\mu>M_{\text {Rep } 1}\right)=\frac{40}{3} g_{3}^{4}+\frac{174}{900} g_{1}^{4}+\frac{3}{2} g_{2}^{4} \\
& \delta \beta_{d}^{(2)}\left(\mu>M_{\text {Rep } 1}\right)=\frac{40}{3} g_{3}^{4}-\frac{6}{900} g_{1}^{4}+\frac{3}{2} g_{2}^{4} \\
& \delta \beta_{e}^{(2)}\left(\mu>M_{\mathrm{Rep} 1}\right)=\frac{66}{100} g_{1}^{4}+\frac{3}{2} g_{2}^{4}
\end{aligned}
$$




$$
\delta \beta_{\lambda}^{(2)}\left(\mu>M_{\mathrm{Rep} 1}\right)=-\frac{6}{250} g_{1}^{4}\left(12 g_{1}^{2}+20 g_{2}^{2}-25 \lambda\right)-\frac{3}{5} g_{2}^{4}\left(4 g_{1}^{2}+20 g_{2}^{2}-25 \lambda\right)
$$

(b) $M_{\text {Rep1 }}>M_{\text {Rep2 }}$

(I) $M_{\mathrm{Rep} 2}<\mu<M_{\mathrm{Rep} 1}$

$\delta b_{i}\left(M_{\text {Rep } 2}<\mu<M_{\text {Rep } 1}\right)=\left(\begin{array}{c}\frac{2}{15} \\ 2 \\ \frac{20}{15}\end{array}\right), \quad \delta m_{i j}\left(M_{\text {Rep } 2}<\mu<M_{\text {Rep } 1}\right)=\left(\begin{array}{ccc}\frac{67}{10000} & \frac{3}{10} & \frac{8}{15} \\ \frac{1}{10} & \frac{245}{10} & 8 \\ \frac{6}{90} & 3 & \frac{76}{3}\end{array}\right)$

$$
\begin{aligned}
& \delta \beta_{u}^{(2)}\left(M_{\mathrm{Rep} 2}<\mu<M_{\mathrm{Rep} 1}\right)=\frac{80}{9} g_{3}^{4}+\frac{58}{900} g_{1}^{4}+\frac{3}{2} g_{2}^{4} \\
& \delta \beta_{d}^{(2)}\left(M_{\mathrm{Rep} 2}<\mu<M_{\mathrm{Rep} 1}\right)=\frac{80}{9} g_{3}^{4}-\frac{2}{900} g_{1}^{4}+\frac{3}{2} g_{2}^{4} \\
& \delta \beta_{e}^{(2)}\left(M_{\mathrm{Rep} 2}<\mu<M_{\mathrm{Rep} 1}\right)=\frac{22}{100} g_{1}^{4}+\frac{3}{2} g_{2}^{4} \\
& \delta \beta_{\lambda}^{(2)}\left(M_{\mathrm{Rep} 2}<\mu<M_{\mathrm{Rep} 1}\right)=-\frac{2}{250} g_{1}^{4}\left(12 g_{1}^{2}+20 g_{2}^{2}-25 \lambda\right)-\frac{3}{5} g_{2}^{4}\left(4 g_{1}^{2}+20 g_{2}^{2}-25 \lambda\right)
\end{aligned}
$$

(II) $\mu>M_{\mathrm{Rep} 1}$

$$
\begin{aligned}
\delta b_{i}\left(\mu>M_{\text {Rep } 1}\right) & =\left(\begin{array}{c}
\frac{4}{10} \\
2 \\
2
\end{array}\right), \quad \delta m_{i j}\left(\mu>M_{\text {Rep } 1}\right)=\left(\begin{array}{ccc}
\frac{6}{100} & \frac{3}{10} & \frac{16}{10} \\
\frac{1}{10} & \frac{245}{10} & 8 \\
\frac{2}{10} & 3 & 38
\end{array}\right) \\
\delta \beta_{u}^{(2)}\left(\mu>M_{\text {Rep } 1}\right) & =\frac{40}{3} g_{3}^{4}+\frac{174}{900} g_{1}^{4}+\frac{3}{2} g_{2}^{4} \\
\delta \beta_{d}^{(2)}\left(\mu>M_{\text {Rep } 1}\right) & =\frac{40}{3} g_{3}^{4}-\frac{6}{900} g_{1}^{4}+\frac{3}{2} g_{2}^{4} \\
\delta \beta_{e}^{(2)}\left(\mu>M_{\text {Rep } 1}\right) & =\frac{66}{100} g_{1}^{4}+\frac{3}{2} g_{2}^{4} \\
\delta \beta_{\lambda}^{(2)}\left(\mu>M_{\text {Rep } 1}\right) & =-\frac{6}{250} g_{1}^{4}\left(12 g_{1}^{2}+20 g_{2}^{2}-25 \lambda\right)-\frac{3}{5} g_{2}^{4}\left(4 g_{1}^{2}+20 g_{2}^{2}-25 \lambda\right)
\end{aligned}
$$

\section{(c) $M_{\text {Rep2 }}>M_{\text {Rep1 }}$}

(I) $M_{\text {Rep1 }}<\mu<M_{\text {Rep2 }}$

$$
\delta b_{i}\left(M_{\mathrm{Rep} 1}<\mu<M_{\mathrm{Rep} 2}\right)=\left(\begin{array}{c}
\frac{4}{15} \\
0 \\
\frac{2}{3}
\end{array}\right), \quad \delta m_{i j}\left(M_{\mathrm{Rep} 1}<\mu<M_{\mathrm{Rep} 2}\right)=\left(\begin{array}{ccc}
\frac{533}{10000} & 0 & \frac{16}{15} \\
0 & 0 & 0 \\
\frac{2}{15} & 0 & \frac{38}{3}
\end{array}\right)
$$

$$
\begin{aligned}
& \delta \beta_{u}^{(2)}\left(M_{\text {Rep } 1}<\mu<M_{\text {Rep } 2}\right)=\frac{40}{9} g_{3}^{4}+\frac{116}{900} g_{1}^{4} \\
& \delta \beta_{d}^{(2)}\left(M_{\text {Rep } 1}<\mu<M_{\text {Rep } 2}\right)=\frac{40}{9} g_{3}^{4}-\frac{4}{900} g_{1}^{4} \\
& \delta \beta_{e}^{(2)}\left(M_{\text {Rep } 1}<\mu<M_{\text {Rep } 2}\right)=\frac{44}{100} g_{1}^{4} \\
& \delta \beta_{\lambda}^{(2)}\left(M_{\text {Rep } 1}<\mu<M_{\text {Rep } 2}\right)=-\frac{4}{250} g_{1}^{4}\left(12 g_{1}^{2}+20 g_{2}^{2}-25 \lambda\right)
\end{aligned}
$$




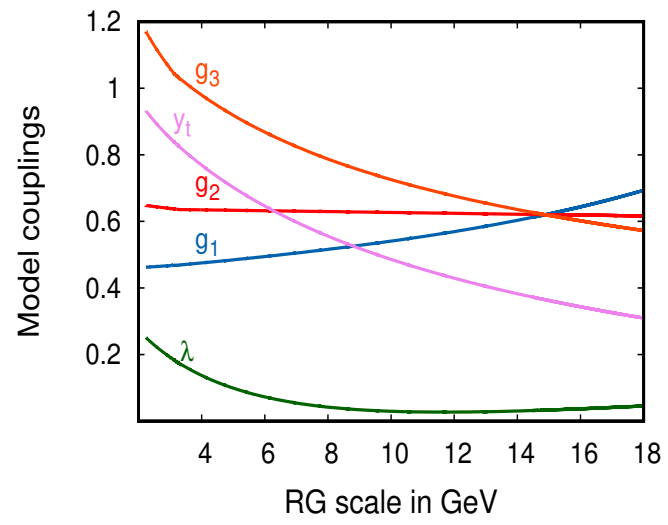

(a)

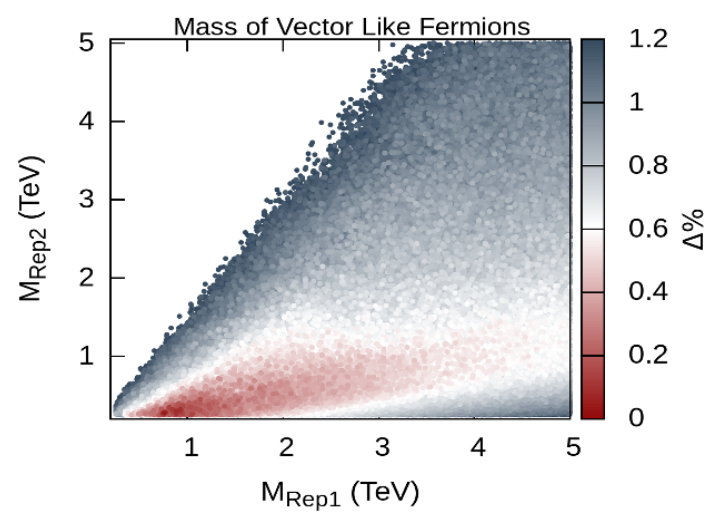

(b)

Figure 9. Model 8: figure (a) Gauge couplings $\left(g_{1}, g_{2}, g_{3}\right)$ unification and Vacuum stability $(\lambda>0)$ plot, considering vector-like fermion in Rep.1 of mass $1.86 \mathrm{TeV}$ and Rep.2 of mass $1.38 \mathrm{TeV}$. Figure (b) Mass range allowed for vector-like fermions in Rep.1 and Rep.2 for gauge unification and vacuum stability.

$$
\begin{aligned}
\text { (II) } \mu>M_{\text {Rep2 }} & \\
\delta b_{i}\left(\mu>M_{\text {Rep } 2}\right) & =\left(\begin{array}{c}
\frac{4}{10} \\
2 \\
2
\end{array}\right), \quad \delta m_{i j}\left(\mu>M_{\text {Rep } 2}\right)=\left(\begin{array}{ccc}
\frac{6}{100} & \frac{3}{10} & \frac{16}{10} \\
\frac{1}{10} & \frac{245}{10} & 8 \\
\frac{2}{10} & 3 & 38
\end{array}\right) \\
\delta \beta_{u}^{(2)}\left(\mu>M_{\text {Rep } 2}\right) & =\frac{40}{3} g_{3}^{4}+\frac{174}{900} g_{1}^{4}+\frac{3}{2} g_{2}^{4} \\
\delta \beta_{d}^{(2)}\left(\mu>M_{\text {Rep } 2}\right) & =\frac{40}{3} g_{3}^{4}-\frac{6}{900} g_{1}^{4}+\frac{3}{2} g_{2}^{4} \\
\delta \beta_{e}^{(2)}\left(\mu>M_{\text {Rep2 }}\right) & =\frac{66}{100} g_{1}^{4}+\frac{3}{2} g_{2}^{4} \\
\delta \beta_{\lambda}^{(2)}\left(\mu>M_{\text {Rep2 } 2}\right) & =-\frac{6}{250} g_{1}^{4}\left(12 g_{1}^{2}+20 g_{2}^{2}-25 \lambda\right)-\frac{3}{5} g_{2}^{4}\left(4 g_{1}^{2}+20 g_{2}^{2}-25 \lambda\right)
\end{aligned}
$$

A sample unification point is shown in figure 8(a), one copy of bottom like vector fermions with degenerate mass of $4.65 \mathrm{TeV}$ and one copy of left handed quark like vector fermion with a mass of $309 \mathrm{GeV}$ is considered. The figure shows unification clearly. The running of $y_{t}$ and $\lambda$ are also shown. The panel figure $8(\mathrm{~b})$ has the mass distibution in Rep1-Rep2 mass plane.

\subsection{Model 8}

This model consist of four copies of $\operatorname{Rep} 1=\left(1,2, \frac{1}{2}\right)$ and one copy of $\operatorname{Rep} 2=(8,1,0)$. The mass range for Rep1 and Rep2 are $(300 \mathrm{GeV}$ to $5 \mathrm{TeV})$ and $(300 \mathrm{GeV}$ to $5 \mathrm{TeV})$ respectively. This representation has been discussed in Model 2 with different number of particles for each represenatation. The difference can been studied with their bound state decay to diphoton channel and dijet, as shown in section 5 . 
For most points in this model vector-like fermions in Rep1 can be degenerate with vector-like fermions in Rep2 $\left(M_{\operatorname{Rep} 1} \sim M_{\mathrm{Rep} 2}\right)$ as shown in figure 9(b). However, there could be points in which either of the $M_{\mathrm{Rep} 1}>M_{\mathrm{Rep} 2}$ and $M_{\mathrm{Rep} 1}<M_{\mathrm{Rep} 2}$ are possible.

The change in the beta functions in the two thresholds are as follows:

(a) $M_{\mathrm{Rep} 1}=M_{\mathrm{Rep} 2}$

(I) $\mu>M_{\text {Rep2 }}=M_{\text {Rep1 }}$

$$
\begin{aligned}
\delta b_{i}\left(\mu>M_{\text {Rep } 1}\right) & =\left(\begin{array}{c}
\frac{16}{10} \\
\frac{24}{9} \\
2
\end{array}\right), \quad \delta m_{i j}\left(\mu>M_{\text {Rep } 1}\right)=\left(\begin{array}{ccc}
\frac{72}{100} & \frac{36}{10} & 0 \\
\frac{12}{10} & \frac{294}{9} & 0 \\
0 & 0 & 48
\end{array}\right) \\
\delta \beta_{u}^{(2)}\left(\mu>M_{\text {Rep } 1}\right) & =\frac{40}{3} g_{3}^{4}+\frac{696}{900} g_{1}^{4}+\frac{4}{2} g_{2}^{4} \\
\delta \beta_{d}^{(2)}\left(\mu>M_{\text {Rep } 1}\right) & =\frac{40}{3} g_{3}^{4}-\frac{24}{900} g_{1}^{4}+\frac{4}{2} g_{2}^{4} \\
\delta \beta_{e}^{(2)}\left(\mu>M_{\text {Rep } 1}\right) & =\frac{264}{100} g_{1}^{4}+\frac{4}{2} g_{2}^{4} \\
\delta \beta_{\lambda}^{(2)}\left(\mu>M_{\text {Rep } 1}\right) & =-\frac{24}{250} g_{1}^{4}\left(12 g_{1}^{2}+20 g_{2}^{2}-25 \lambda\right)-\frac{4}{5} g_{2}^{4}\left(4 g_{1}^{2}+20 g_{2}^{2}-25 \lambda\right)
\end{aligned}
$$

(b) $M_{\text {Rep1 }}>M_{\text {Rep2 }}$

(I) $M_{\mathrm{Rep} 2}<\mu<M_{\mathrm{Rep} 1}$

$$
\begin{aligned}
\delta b_{i}\left(M_{\mathrm{Rep} 2}<\mu<M_{\mathrm{Rep} 1}\right) & =\left(\begin{array}{c}
0 \\
0 \\
2
\end{array}\right), \quad \delta m_{i j}\left(M_{\mathrm{Rep} 2}<\mu<M_{\mathrm{Rep} 1}\right)=\left(\begin{array}{ccc}
0 & 0 & 0 \\
0 & 0 & 0 \\
0 & 0 & 48
\end{array}\right) \\
\delta \beta_{u}^{(2)}\left(M_{\mathrm{Rep} 2}<\mu<M_{\mathrm{Rep} 1}\right) & =\frac{40}{3} g_{3}^{4} \\
\delta \beta_{d}^{(2)}\left(M_{\mathrm{Rep} 2}<\mu<M_{\mathrm{Rep} 1}\right) & =\frac{40}{3} g_{3}^{4} \\
\delta \beta_{e}^{(2)}\left(M_{\mathrm{Rep} 2}<\mu<M_{\mathrm{Rep} 1}\right) & =0 \\
\delta \beta_{\lambda}^{(2)}\left(M_{\mathrm{Rep} 2}<\mu<M_{\mathrm{Rep} 1}\right) & =0
\end{aligned}
$$

(II) $\mu>M_{\mathrm{Rep} 1}$

$$
\begin{aligned}
\delta b_{i}\left(\mu>M_{\text {Rep } 1}\right) & =\left(\begin{array}{c}
\frac{16}{10} \\
\frac{24}{9} \\
2
\end{array}\right), \quad \delta m_{i j}\left(\mu>M_{\text {Rep } 1}\right)=\left(\begin{array}{ccc}
\frac{72}{100} & \frac{36}{10} & 0 \\
\frac{12}{10} & \frac{294}{9} & 0 \\
0 & 0 & 48
\end{array}\right) \\
\delta \beta_{u}^{(2)}\left(\mu>M_{\text {Rep } 1}\right) & =\frac{40}{3} g_{3}^{4}+\frac{696}{900} g_{1}^{4}+\frac{4}{2} g_{2}^{4} \\
\delta \beta_{d}^{(2)}\left(\mu>M_{\text {Rep } 1}\right) & =\frac{40}{3} g_{3}^{4}-\frac{24}{900} g_{1}^{4}+\frac{4}{2} g_{2}^{4} \\
\delta \beta_{e}^{(2)}\left(\mu>M_{\text {Rep } 1}\right) & =\frac{264}{100} g_{1}^{4}+\frac{4}{2} g_{2}^{4} \\
\delta \beta_{\lambda}^{(2)}\left(\mu>M_{\text {Rep } 1}\right) & =-\frac{24}{250} g_{1}^{4}\left(12 g_{1}^{2}+20 g_{2}^{2}-25 \lambda\right)-\frac{4}{5} g_{2}^{4}\left(4 g_{1}^{2}+20 g_{2}^{2}-25 \lambda\right)
\end{aligned}
$$


(c) $M_{\text {Rep2 }}>M_{\text {Rep1 }}$

(I) $M_{\mathrm{Rep} 1}<\mu<M_{\mathrm{Rep} 2}$

$$
\begin{aligned}
\delta b_{i}\left(M_{\text {Rep } 1}<\mu<M_{\text {Rep } 2}\right) & =\left(\begin{array}{c}
\frac{16}{10} \\
\frac{24}{9} \\
0
\end{array}\right), \quad \delta m_{i j}\left(M_{\text {Rep } 1}<\mu<M_{\text {Rep } 2}\right)=\left(\begin{array}{ccc}
\frac{72}{100} & \frac{36}{10} & 0 \\
\frac{12}{10} & \frac{294}{9} & 0 \\
0 & 0 & 0
\end{array}\right) \\
\delta \beta_{u}^{(2)}\left(M_{\text {Rep } 1}<\mu<M_{\text {Rep } 2}\right) & =\frac{696}{900} g_{1}^{4}+\frac{4}{2} g_{2}^{4} \\
\delta \beta_{d}^{(2)}\left(M_{\text {Rep } 1}<\mu<M_{\text {Rep } 2}\right) & =-\frac{24}{900} g_{1}^{4}+\frac{4}{2} g_{2}^{4} \\
\delta \beta_{e}^{(2)}\left(M_{\text {Rep } 1}<\mu<M_{\text {Rep } 2}\right) & =\frac{264}{100} g_{1}^{4}+\frac{4}{2} g_{2}^{4} \\
\delta \beta_{\lambda}^{(2)}\left(M_{\text {Rep } 1}<\mu<M_{\text {Rep } 2}\right) & =-\frac{24}{250} g_{1}^{4}\left(12 g_{1}^{2}+20 g_{2}^{2}-25 \lambda\right)-\frac{4}{5} g_{2}^{4}\left(4 g_{1}^{2}+20 g_{2}^{2}-25 \lambda\right)
\end{aligned}
$$

(II) $\mu>M_{\operatorname{Rep} 2}$

$$
\begin{aligned}
\delta b_{i}\left(\mu>M_{\text {Rep } 2}\right) & =\left(\begin{array}{c}
\frac{16}{10} \\
\frac{24}{9} \\
2
\end{array}\right), \quad \delta m_{i j}\left(\mu>M_{\text {Rep } 2}\right)=\left(\begin{array}{ccc}
\frac{72}{100} & \frac{36}{10} & 0 \\
\frac{12}{10} & \frac{294}{9} & 0 \\
0 & 0 & 48
\end{array}\right) \\
\delta \beta_{u}^{(2)}\left(\mu>M_{\text {Rep } 2}\right) & =\frac{40}{3} g_{3}^{4}+\frac{696}{900} g_{1}^{4}+\frac{4}{2} g_{2}^{4} \\
\delta \beta_{d}^{(2)}\left(\mu>M_{\text {Rep } 2}\right) & =\frac{40}{3} g_{3}^{4}-\frac{24}{900} g_{1}^{4}+\frac{4}{2} g_{2}^{4} \\
\delta \beta_{e}^{(2)}\left(\mu>M_{\text {Rep } 2}\right) & =\frac{264}{100} g_{1}^{4}+\frac{4}{2} g_{2}^{4} \\
\delta \beta_{\lambda}^{(2)}\left(\mu>M_{\text {Rep2 } 2}\right) & =-\frac{24}{250} g_{1}^{4}\left(12 g_{1}^{2}+20 g_{2}^{2}-25 \lambda\right)-\frac{4}{5} g_{2}^{4}\left(4 g_{1}^{2}+20 g_{2}^{2}-25 \lambda\right)
\end{aligned}
$$

A sample unification point is shown in figure 9 (a), four copies of lepton like vector fermions with degenerate mass of $1.86 \mathrm{TeV}$ and one copy of gluion like vector fermion with a mass of $1.38 \mathrm{TeV}$ is considered. The figure shows unification clearly. The running of $y_{t}$ and $\lambda$ are also shown. The panel figure 9(b) has the mass distibution in Rep1-Rep2 mass plane.

\subsection{Model 9}

This model consist of three copies of $\operatorname{Rep} 1=(1,3,0)$ and six copies of $\operatorname{Rep} 2=\left(3,1, \frac{1}{3}\right)$. The mass range for Rep1 and Rep2 are $(1.1 \mathrm{TeV}$ to $5 \mathrm{TeV})$ and $(250 \mathrm{GeV}$ to $1.8 \mathrm{TeV})$ respectively. This representation has been discussed in Model 3 with different number of particle for each represenatation. The difference can been studied with their bound state decay to diphoton channel and dijet, as shown in section 5. In the model, $M_{\text {Rep1 }}$ is greater than $M_{\text {Rep2 }}$. The change in the beta functions in the two thresholds are as follows: 


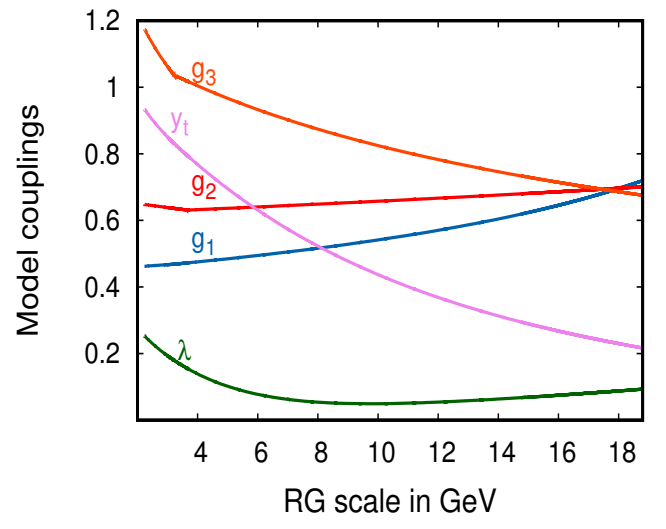

(a)

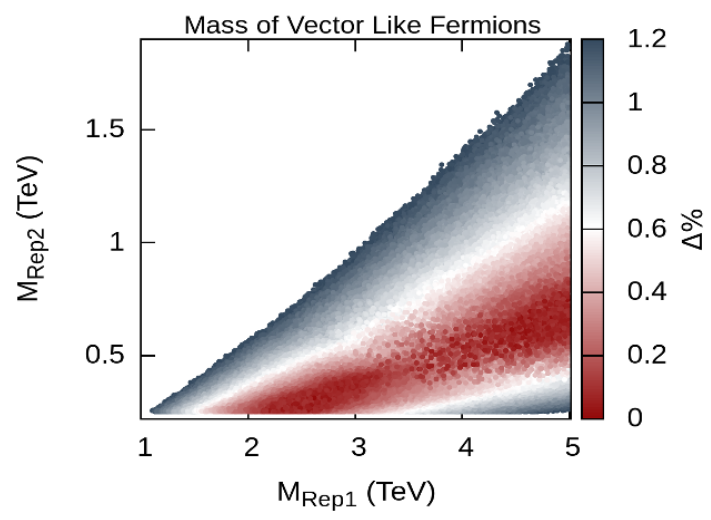

(b)

Figure 10. Model 9: figure (a) Gauge couplings $\left(g_{1}, g_{2}, g_{3}\right)$ unification and Vacuum stability $(\lambda>0)$ plot, considering vector-like fermion in Rep.1 of mass $4.6 \mathrm{TeV}$ and Rep.2 of mass $1.6 \mathrm{TeV}$. Figure (b) Mass range allowed for vector-like fermions in Rep.1 and Rep.2 for gauge unification and vacuum stability.

(I) $M_{\text {Rep2 }}<\mu<M_{\text {Rep1 }}$

$\delta b_{i}\left(M_{\text {Rep } 2}<\mu<M_{\text {Rep } 1}\right)=\left(\begin{array}{c}\frac{16}{10} \\ 0 \\ 4\end{array}\right), \quad \delta m_{i j}\left(M_{\text {Rep } 2}<\mu<M_{\text {Rep } 1}\right)=\left(\begin{array}{ccc}\frac{32}{100} & 0 & \frac{64}{10} \\ 0 & 0 & 0 \\ \frac{8}{10} & 0 & 76\end{array}\right)$

$\delta \beta_{u}^{(2)}\left(M_{\mathrm{Rep} 2}<\mu<M_{\mathrm{Rep} 1}\right)=\frac{240}{9} g_{3}^{4}+\frac{696}{900} g_{1}^{4}$

$\delta \beta_{d}^{(2)}\left(M_{\mathrm{Rep} 2}<\mu<M_{\mathrm{Rep} 1}\right)=\frac{240}{9} g_{3}^{4}-\frac{24}{900} g_{1}^{4}$

$\delta \beta_{e}^{(2)}\left(M_{\mathrm{Rep} 2}<\mu<M_{\mathrm{Rep} 1}\right)=\frac{264}{100} g_{1}^{4}$

$\delta \beta_{\lambda}^{(2)}\left(M_{\mathrm{Rep} 2}<\mu<M_{\mathrm{Rep} 1}\right)=-\frac{24}{250} g_{1}^{4}\left(12 g_{1}^{2}+20 g_{2}^{2}-25 \lambda\right)$

(II) $\mu>M_{\text {Rep1 }}$

$$
\begin{aligned}
\delta b_{i}\left(\mu>M_{\text {Rep } 1}\right) & =\left(\begin{array}{c}
\frac{16}{10} \\
4 \\
4
\end{array}\right), \quad \delta m_{i j}\left(\mu>M_{\text {Rep } 1}\right)=\left(\begin{array}{ccc}
\frac{32}{100} & 0 & \frac{64}{10} \\
0 & 64 & 0 \\
\frac{8}{10} & 0 & 76
\end{array}\right) \\
\left.\delta \beta_{u}^{(2)} \mu>M_{\text {Rep } 1}\right) & =\frac{240}{9} g_{3}^{4}+\frac{696}{900} g_{1}^{4}+\frac{6}{2} g_{1}^{4} \\
\delta \beta_{d}^{(2)}\left(\mu>M_{\text {Rep } 1}\right) & =\frac{240}{9} g_{3}^{4}-\frac{24}{900} g_{1}^{4}+\frac{6}{2} g_{1}^{4} \\
\delta \beta_{e}^{(2)}\left(\mu>M_{\text {Rep } 1}\right) & =\frac{264}{100} g_{1}^{4}+\frac{6}{2} g_{1}^{4} \\
\delta \beta_{\lambda}^{(2)}\left(\mu>M_{\text {Rep } 1}\right) & =-\frac{24}{250} g_{1}^{4}\left(12 g_{1}^{2}+20 g_{2}^{2}-25 \lambda\right)-\frac{6}{5} g_{2}^{4}\left(4 g_{1}^{2}+20 g_{2}^{2}-25 \lambda\right)
\end{aligned}
$$


A sample unification point is shown in figure 10(a), three copies of weak-isospin triplet vector-like fermions with degenerate mass of $4.6 \mathrm{TeV}$ and six copies of bottom like vector fermion with a mass of $1.6 \mathrm{GeV}$ is considered. The figure shows unification clearly. The running of $y_{t}$ and $\lambda$ are also shown. The panel figure 10(b) has the mass distibution in Rep1-Rep2 mass plane.

\section{Collider signature of minimal vector-like fermion models}

The models listed in table 2 have several exotic states lying close to electroweak scale, which can be probed at LHC. Models have exotic lepton like states (uncoloured) mostly in doublet, triplet and fourplet representation of SU(2). These states are produced at the LHC through Drell-Yan process and typically have cross-section of the order $10 \mathrm{fb}$ [74] (roughly slepton production or exotic lepton production). These particles decay through Yukawa interaction to lighter SM leptons. In the limit of vanishing Yukawa couplings, these particles can manifest as missing energy and disappearing charge track at LHC and limits from monojets and disappearing tracks could apply to our model. The LHC at $14 \mathrm{TeV}$ with integrated luminosity $3000 \mathrm{fb}^{-1}$ is only sensitive to mass of order $400 \mathrm{GeV}$ [75]. In the following we will concentrate on the strongly interacting exotic sector; which appears in all the successful models.

\subsection{Decay operators}

The models tabulated in the above has exotic fields and some of these fields don't have renormalisation level decay operators. These fields are (i) $\left(6,1, \frac{1}{3}\right)$, (ii) $\left(6,1, \frac{2}{3}\right)$ and (iii) $(8,1,0)$. Now question is whether we can have higher dimensional operators or not. Note that if there exists any higher dimensional operator then there must be some new fields which got integrated out in some higher scales. Now this scale has to be high (close to the GUT scale) as otherwise unification will be disturbed. These higher dimensional operators are suppressed as

$$
\frac{\mathcal{O}}{\Lambda^{\operatorname{dim}(\mathcal{O})-4}},
$$

where $\operatorname{dim}(\mathcal{O})$ is the dimension of the operator $\mathcal{O}$. Six-dimensional operators are suppressed by square of the GUT scale and thus life-time of the particle is expected to be High $\left(\sim 10^{33}\right.$ years). Thus we are focusing only on the five dimensional operators. Any five dimensional operator for decay of such particle must have the forms:

(1) Exotic field $\times$ a standard model fermion $\times$ Higgs $\times$ Higgs

(2) Exotic field $\times$ a standard model fermion $\times$ Gauge boson $\times$ Gauge boson,

where in the place of the Higgs and SM fermions fields one can use their conjugate fields. Thus colour charge of the exotic field has to be neutralized by SM fermion to form a five dimensional operator involving the Higgs. In the SM, there is no such field and hence possibility (1) is not possible. For the second case, colour representation of the exotic times that of the SM fermion field must transform as any one of 1, 8, 10 and 27 dimensional representation. However we don't have SM field with above representation hence, this 
second possibility is also ruled out. These exotic fields can form a bound state and in the next subsection we'll discuss this in details.

\subsection{Formalism for bound state}

In this section we investigate the possibility of producing bound states of the colour vectorlike fermions. The idea of bound state has been studied, in understanding bottom and charm quark through their bound states. For the formation of bound state, we assume the new vector-like fermion $(\psi)$ is long lived so that it has time to form a bound state prior to decaying. This condition is easily satisfied in our case, as the Yukawa coupling between the new vector-like fermions and SM particle is assumed to be negligible. The bound state formalism has been studied in [76, 77], where they focus on pair-produced colour particle Beyond the Standard Model by the observation of diphoton, dijet etc. resonances arising from QCD bound state.

We assume that the only interaction that contribute to the production of bound state is the Standard Model SU(3) colour gauge interaction. We estimate the annihilation rates and parton-level cross-section at leading order, along with NLO MSTW parton distribution functions [78], to compute the LHC signals for $\sqrt{s}=8 \mathrm{TeV}, 13 \mathrm{TeV}$ and $14 \mathrm{TeV}$ evaluated at scale $m_{\psi}$. The production cross section of colour singlet spin zero bound state from constituent vector-like fermion with colour representation $3,6,8$ are shown in figure 11 and figure 14. As pointed out in ref. [79], NLO corrections to cross-section can increase the diphoton resonance arising from stoponium by $25 \%$. Therefore, large uncertainties are expected in our result of factor of two or so. This still can allow us to constraints minimal vector-like fermion model.

Further uncertainty in our results arises because of limits extracted from ATLAS and CMS result, which is obtained for a fixed spin and production channel. Signal shape have some dependence on the acceptance, intrinsic width and whether a jet is due to parton-level gluon or quark, this adds to some uncertainties.

A pair of $\psi \bar{\psi}$ near threshold can form a QCD bound state, which we defined as $\mathcal{O}$. If the decay width of $\mathcal{O}$ is smaller than its respective binding energy, it can be observed as a resonance which annihilates to SM particles. For particles $(\psi)$ of mass $m_{\psi} \gg \Lambda_{Q C D}$, the Bohr radius of relevant bound state is much smaller than the QCD scale and the velocity of its constituents is non relativistic, we can estimate bound state as modified hydrogenic approximation. For a particle $\psi$ in the colour representation $R$, the potential between $\psi$ and $\bar{\psi}$ depends on the colour representation $\mathcal{R}$ of the $\psi \bar{\psi}$ pair through the casimirs of $R$ and $\mathcal{R}$ as

$$
V(r)=-C \frac{\bar{\alpha}_{s}}{r}, \quad C=C(R)-\frac{1}{2} C(\mathcal{R})
$$

where $\bar{\alpha}_{s}$ is defined as the running coupling at the scale of the average distance between the two particle in the corresponding hydrogenic state, which is order of the Bohr radius $a_{0}=2 /\left(C \bar{\alpha}_{s} m_{\psi}\right)$ (for which we used ref. [80]). The binding energy of the wave functions at the origin for the ground state are given by

$$
E_{b}=-\frac{1}{4} C^{2} \bar{\alpha}_{s}^{2} m_{\psi}, \quad|\psi(0)|^{2} \equiv \frac{1}{4 \pi}|R(0)|^{2}=\frac{C^{3} \bar{\alpha}_{s}^{3} m_{\psi}^{3}}{8 \pi}
$$


The quantum number of $\psi$ determines the production as well as the decay modes of bound state particle $\mathcal{O}$. The cross-section for the bound state $\mathcal{O}$ to be produced by initial-state partons $x$ and $y$ is given as

$$
\hat{\sigma}_{x y \rightarrow \mathcal{O}}(\hat{s})=\frac{8 \pi}{m_{\psi}} \frac{\hat{\sigma}_{x y \rightarrow \psi \bar{\psi}}^{\text {free }}(\hat{s})}{\beta(\hat{s})}|\psi(0)|^{2} 2 \pi \delta\left(\hat{s}-M^{2}\right)
$$

where $M=2 m_{\psi}+E_{b}$ is the mass of the bound state, $\beta(\hat{s})$ is the velocity of $\psi$ or $\bar{\psi}$ in CM frame. The production cross-section of any narrow resonance $\mathcal{O}$ of mass $M$ and spin $J$ from parton $x$ and $y$, and the decay rate of bound state to $x$ and $y$, are related by

$$
\hat{\sigma}_{x y \rightarrow \mathcal{O}}=\frac{2 \pi(2 J+1) d_{\mathcal{O}}(\mathcal{R})}{D_{x} D_{y}} \frac{\Gamma_{\mathcal{O} \rightarrow x y}}{M} 2 \pi \delta\left(\hat{s}-M^{2}\right) \quad(\times 2 \text { for } \mathrm{x}=\mathrm{y})
$$

where $D_{\mathcal{O}}$ denotes the colour representation of particle $\mathcal{O}$.

In the next subsection we will strict ourself to study the colour singlet and spin zero $(\mathrm{J}=0)$ bound state system. Assuming the production cross-section of $\psi \bar{\psi}$ is dominated by gluon fusion. The gluon fusion partonic production cross-section of bound state is given by

$$
\hat{\sigma}_{g g \rightarrow \mathcal{O}}=\frac{\pi^{2}}{8} \frac{\Gamma_{\mathcal{O} \rightarrow g g}}{M} \delta\left(\hat{s}-M^{2}\right)
$$

Depending on the quantum number of $\psi$, bound state $\mathcal{O}$ can decay to diphoton, dijet, $Z \gamma, Z Z$ and $W^{+} W^{-}$channels. The production of preceding pair events produced in proton-proton collisions in LHC can be predicted as $\sigma(p p \rightarrow \mathcal{O}) \times B R\left(\mathcal{O} \rightarrow X_{1} X_{2}\right)$.

Here we will identify the channels in which the bound state resonance would be most easily measurable and compute the corresponding cross-section as a function of the mass, colour representation and charge of the constituent particles. The promising final states that we analyzed are diphoton and dijet channels. In the case of SU(2) multiplet the large mass splitting is constrained by Electroweak precision test, which modifies the oblique parameter T and S [38], hence we have analysed our results in degenerate mass scenario.

\subsection{Signals}

\subsection{1 $\gamma \gamma, Z Z, Z \gamma, W^{+} W^{-}$channel}

Any spin half particle can be produced in pairs (in gg collisions) in an S-wave $J=0$ colour singlet bound state, which can decay as typically narrow $\gamma \gamma, Z Z, Z \gamma$ resonance. The decay width of the $\gamma \gamma, Z Z, Z \gamma$ signal due to spin $J=0$ bound state is given as [81]

$$
\begin{aligned}
\Gamma\left(\mathcal{O}_{J=0}^{\mathcal{R}} \rightarrow \gamma \gamma\right) & =\frac{Q^{4} C(R)^{3} d_{R}}{2} \alpha^{2} \bar{\alpha}_{s}^{3} m_{\psi} \\
\Gamma\left(\mathcal{O}_{J=0}^{\mathcal{R}} \rightarrow \gamma Z\right) & =\frac{Q^{2} C(R)^{3} d_{R}}{\sin ^{2} \theta_{W} \cos ^{2} \theta_{W}}\left(1-R_{Z}\right) v^{2} \alpha^{2} \bar{\alpha}_{s}^{3} m_{\psi} \\
\Gamma\left(\mathcal{O}_{J=0}^{\mathcal{R}} \rightarrow Z Z\right) & =\frac{C(R)^{3} d_{R}}{2 \sin ^{4} \theta_{W} \cos ^{4} \theta_{W}} \frac{\beta_{Z}^{3}}{\left(1-2 R_{Z}\right)} v^{4} \alpha^{2} \bar{\alpha}_{s}^{3} m_{\psi}
\end{aligned}
$$

where $v=\frac{1}{2}\left(T_{3 L}+T_{3 R}\right)-Q \sin ^{2} \theta_{W}, T_{3 L, 3 R}$ is the third component of the weak isospin for the left and right handed state of the fermion, $Q$ is the charge of particle, $R_{Z}=M_{Z} / M$ and $\beta_{Z}=\sqrt{1-4 R_{Z}}$. 
Model No. 4 of minimal fermion model contains constituent of vector-like fermion $(3,2,1 / 6)$ with $\mathrm{SU}(2)$ doublet. This can also decay to $W^{+} W^{-}$channel, which is comparable to $\gamma \gamma$ channel. The decay width for $W^{+} W^{-}$is given as [81],

$$
\Gamma\left(\mathcal{O}_{J=0}^{\mathcal{R}} \rightarrow W^{+} W^{-}\right)=\frac{3 \alpha^{2} \beta_{W}^{3}}{16 \sin ^{4} \theta_{W}} \frac{1}{\left(1-2 R_{W}\right)^{2}} \bar{\alpha}_{s}^{3} m_{\psi}
$$

where $R_{W}=M_{W} / M, \beta_{W}=\sqrt{1-4 R_{W}}$.

The branching fraction of the isoweak singlet fermions which satisfied the gauge coupling unification and vacuum stability are tabulated in table 3 .

Model No. 4 with vector-like fermion constituent $(3,2,1 / 6)$, can decay to $g g$ or $\gamma \gamma, Z \gamma$, $Z Z$ and $W W$ channels. With charge $\mathrm{Q}=-1 / 3$ the branching fraction at mass $m_{\psi}=1 \mathrm{TeV}$ is $93.55 \%, 2.80 \times 10^{-2} \%, 0.49 \%, 2.13 \%$ and $3.79 \%$ respectively and for $\mathrm{Q}=2 / 3$ is $93.49 \%$, $0.44 \%, 1.31 \%, 0.95 \% 3.79 \%$ respectively. We observed that in a large isoweak SU(2) represenatation the total decay width can be larger than its width into gg.

Both ATLAS and CMS have performed a search of resonant production of photon pairs for scalar particle $(\mathrm{J}=0)$. ATLAS [82] analysis is based on data corresponding to an integrated luminosity of $15.4 \mathrm{fb}^{-1}$ at $\sqrt{s}=13$ recorded in 2015 and 2016. CMS [83] data sample correspond to luminosity $12.9 \mathrm{fb}^{-1}$ at $\sqrt{s}=13$ in 2016 , combined statistically with the previous data of 2012 and 2015 at $\sqrt{s}=8$ and $\sqrt{s}=13$ respectively, with luminosity of 19.7 and $3.3 \mathrm{fb}^{-1}$.

\subsubsection{Dijet channel}

S-wave bound state with spin $J=0$ can be produced via $g g \rightarrow \mathcal{O}$ and annihilating mostly to $g g$. For $\mathrm{j}=1 / 2$ there is also a comparable contribution from S-wave $J=1$ colour octet bound states produced via $q \bar{q} \rightarrow \mathcal{O}$ and annihilating to $q \bar{q}$, which we will not discuss here.

The decay width of $g g$ signal due to spin $J=0$ colour singlet bound state is,

$$
\Gamma\left(\mathcal{O}_{J=0}^{\mathcal{R}=1} \rightarrow g g\right)=\frac{C(R)^{5} d_{R}}{32} \alpha_{s}^{2} \bar{\alpha}_{s}^{3} m_{\psi}
$$

( $\times 2$ for Complex Representation of constituent fermion).

Search for narrow resonances decaying to dijet final states in proton-proton collision has been performed by the ATLAS and CMS collaborations using the LHC run data at $\sqrt{s}$ $8 \mathrm{TeV}$ as well as $13 \mathrm{TeV}$. CMS [84] study has been performed with integrated luminosity $18.8 \mathrm{fb}^{-1}$ at $\sqrt{s}=8 \mathrm{TeV}$ using a novel technique called data scouting. ATLAS [85] has studied with $\sqrt{s}=8 \mathrm{TeV}$ using full integrated luminosity of $20.3 \mathrm{fb}^{-1}$ masses upto $4.5 \mathrm{TeV}$. In run-II, ATLAS [86] with centre-of-mass energy $\sqrt{s}=13$ has studied the dijet search using the data collected in 2015 and 2016 with luminosity $3.5 \mathrm{fb}^{-1}$ and $33.5 \mathrm{fb}^{-1}$ respectively and CMS [87] has presented a data with luminosity $36 \mathrm{fb}^{-1}$ considering masses above $600 \mathrm{GeV}$.

\subsection{Limits on signals from CMS and ATLAS}

In next section we examine the constraints on masses of bound state from dijet and diphoton bounds considering one copy of constituent vector-like fermions. We have used the recent limits of ATLAS and CMS for diphoton resonance at centre of energy $\sqrt{s}=13 \mathrm{TeV}$ from 


\begin{tabular}{|c|c|c|c|c|c|}
\hline Fermion & $\mathcal{O}$ & \multicolumn{4}{|c|}{ Branching Fraction $\times 100$} \\
\hline & & $B R(\mathcal{O} \rightarrow g g) \times 100$ & $B R(\mathcal{O} \rightarrow \gamma \gamma) \times 100$ & $B R(\mathcal{O} \rightarrow \gamma Z) \times 100$ & $B R(\mathcal{O} \rightarrow Z Z) \times 100$ \\
\hline$(6,1,1 / 3)$ & 1 & 99.99 & $4.80 \times 10^{-3}$ & $2.79 \times 10^{-3}$ & $4.98 \times 10^{-4}$ \\
\hline$(6,1,2 / 3)$ & 1 & 99.87 & $7.67 \times 10^{-2}$ & $4.45 \times 10^{-2}$ & $7.95 \times 10^{-3}$ \\
\hline$(8,1,0)$ & 1 & 100 & - & - & - \\
\hline$(3,1,1 / 3)$ & 1 & 99.95 & $2.99 \times 10^{-2}$ & $1.74 \times 10^{-2}$ & $3.11 \times 10^{-3}$ \\
\hline$(3,1,2 / 3)$ & 1 & 99.19 & 0.47 & 0.27 & $4.94 \times 10^{-2}$ \\
\hline
\end{tabular}

Table 3. Branching fraction for Bound state of $J=0$, colour representation singlet at mass of $m_{\psi}=1 \mathrm{TeV}$.

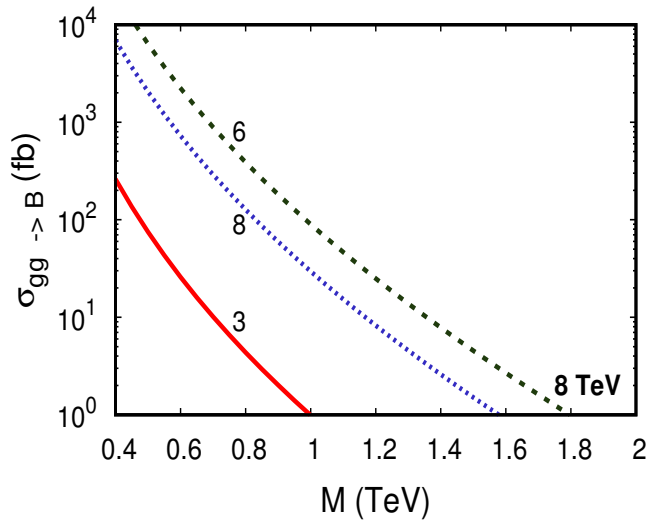

(a)

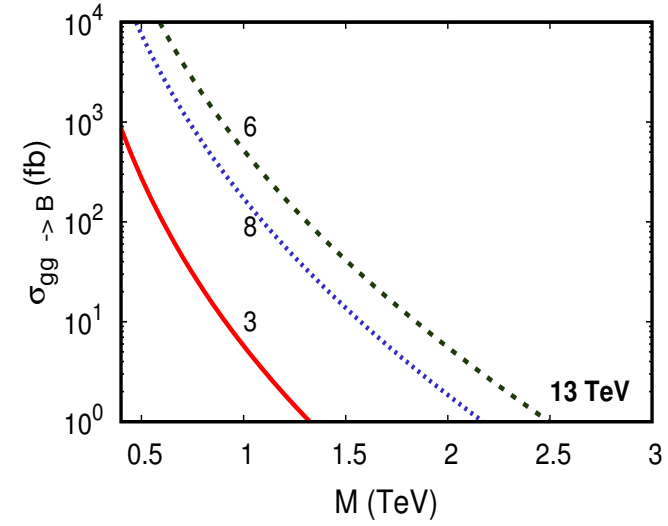

(b)

Figure 11. Cross section of Bound State for Representation $\mathcal{R}=1$ and $J=0$, from constituent particle of Representation $R=3,6,8$ with respect to mass of bound state. The left figure corespond to $\sqrt{s}=8 \mathrm{TeV}$ and right at $\sqrt{s}=13 \mathrm{TeV}$.

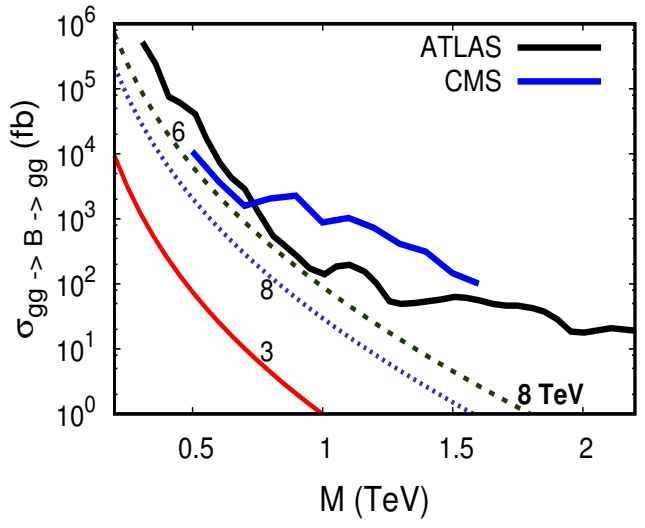

(a)

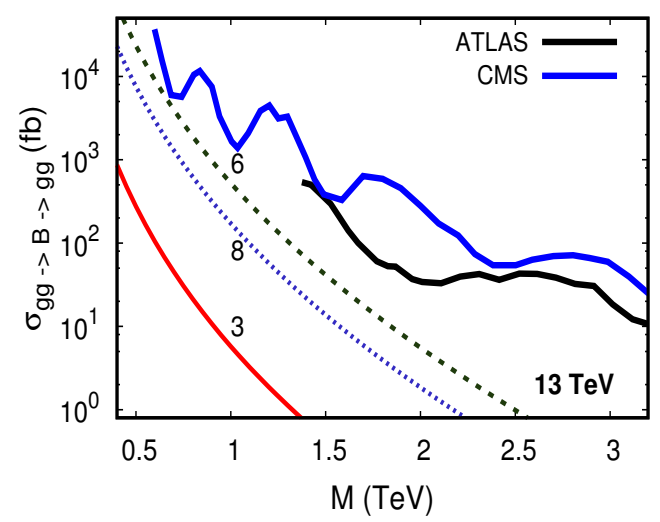

(b)

Figure 12. Cross section of Dijet events at $\sqrt{s}=8 \mathrm{TeV}$ (left) and $\sqrt{s}=13 \mathrm{TeV}$ (right) for Bound State of Representation $\mathcal{R}=1$ and $J=0$, from constituent particle of Representation $R=3,6,8$. Limits from ATLAS $8 \mathrm{TeV}$ and $13 \mathrm{TeV}$ are shown in thick black and CMS $8 \mathrm{TeV}$ and $13 \mathrm{TeV}$ are shown in thick blue. 


\begin{tabular}{|c|c|c|c|}
\hline Model & Representation & Diphoton $(\mathrm{GeV})$ & Dijet $(\mathrm{GeV})$ \\
\hline Model1 & $\operatorname{Rep2} \sim 1(6,1,1 / 3)$ & 220 & - \\
\hline Model2 & $\operatorname{Rep2} \sim 2(8,1,0)$ & - & - \\
\hline Model3 & $\operatorname{Rep2} \sim 4(3,1,1 / 3)$ & 150 & - \\
\hline \multirow{2}{*}{ Model4 } & $\operatorname{Rep1} \sim 2(3,1,2 / 3)$ & 300 & - \\
\cline { 2 - 4 } & $\operatorname{Rep2} \sim 2(3,2,1 / 6)$ & 300 & - \\
\hline Model5 & $\operatorname{Rep2~} 1(6,1,2 / 3)$ & 390 & - \\
\hline Model6 & $\operatorname{Rep2~} \sim 2(6,1,2 / 3)$ & 450 & - \\
\hline \multirow{2}{*}{ Model7 } & $\operatorname{Rep1} \sim 1(3,1,1 / 3)$ & - & - \\
\cline { 2 - 4 } & $\operatorname{Rep2} \sim 1(3,2,1 / 6)$ & 220 & - \\
\hline Model8 & $\operatorname{Rep2} \sim 1(8,1,0)$ & - & - \\
\hline Model9 & $\operatorname{Rep2} \sim 6(3,1,1 / 3)$ & 200 & - \\
\hline
\end{tabular}

Table 4. Lower bounds on masses of vector-like fermions $\left(m_{\psi}=M / 2\right)$ from dijet and diphoton events.

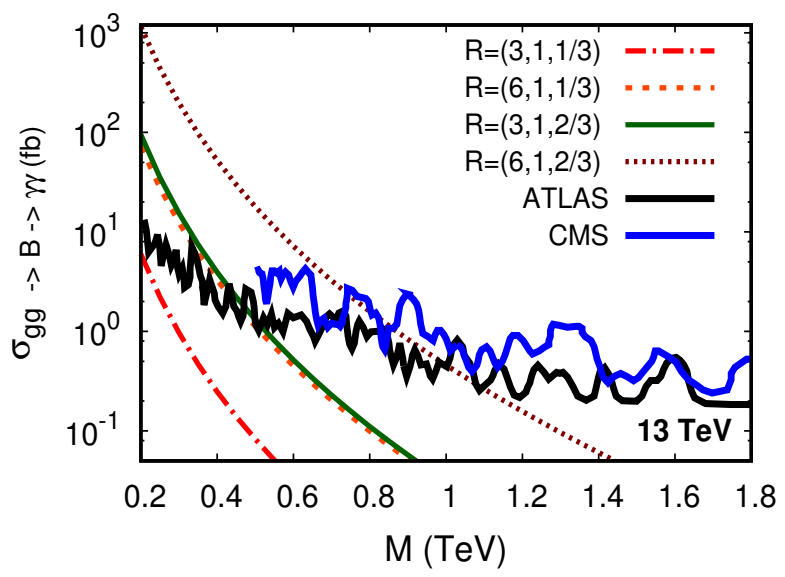

Figure 13. Cross section of diphoton event w.r.t. bound state mass at $\sqrt{s}=13 \mathrm{TeV}$ for Bound State of Representation $\mathcal{R}=1$ and $J=0$ from constituent particle of Color Representation $R=3$, 6. The red line(dash dot) shows the fermion with $R=3$ and $\mathrm{Q}=1 / 3$, green line(solid) correspond to $R=3$ and $\mathrm{Q}=2 / 3$, purple line(dotted) shows the fermion with $R=6$ and $\mathrm{Q}=2 / 3$ and orange line(dashed) shows the $R=6$ and $\mathrm{Q}=1 / 3$ fermion. Limits are from ATLAS $13 \mathrm{TeV}$ black line and CMS $13 \mathrm{TeV}$ blue line.

2015 and as well as 2016 data. Dijet bounds has been considered for centre of energy $\sqrt{s}=8$ and $13 \mathrm{TeV}$ from both ATLAS and CMS.

As we have $\mathrm{n}$ number of copies of vector-like fermions described in the in section 4 for two fermions representation, we will give the exclusion limits of vector-like fermion particle occurring in different models with $\mathrm{n}$ number of copies in the table 4. 


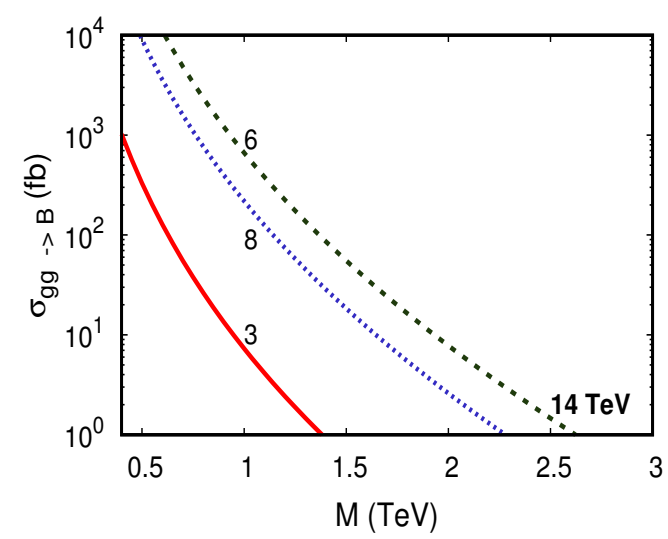

Figure 14. Cross section of Bound State w.r.t. bound state mass at $\sqrt{s}=14 \mathrm{TeV}$ for Representation $\mathcal{R}=1$ and $J=0$, from constituent particle of Representation $R=3,6,8$.

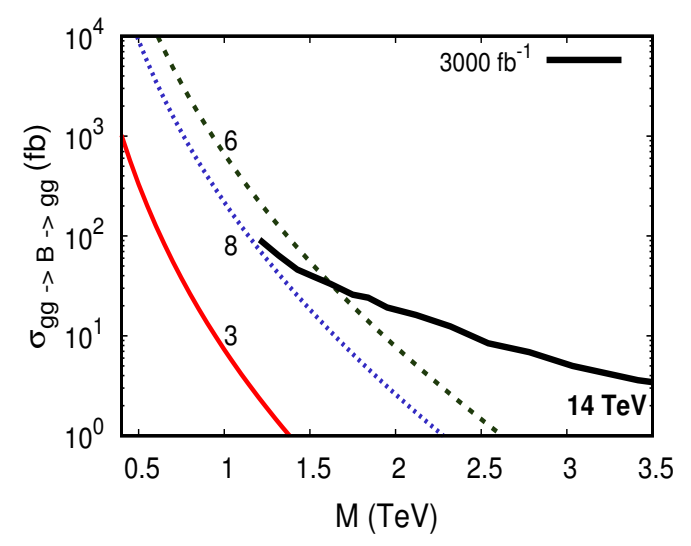

Figure 15. Cross section of Dijet events at $\sqrt{s}$ $=14 \mathrm{TeV}$ for Bound State for Representation $\mathcal{R}=1$ and $J=0$, from constituent particle of Representation $R=3,6,8$ w.r.t. bound state mass. Future limits from $14 \mathrm{TeV}$ at $3000 \mathrm{fb}^{-1}$ is shown in thick black line.

\subsubsection{Dijet bounds}

In figure 12(a)(b) we present the $\sigma(p p \rightarrow \mathcal{O}) \times B R(\mathcal{O} \rightarrow g g)$ as a function of the mass of the $\mathcal{O}$ resonance considering one copy of constituent vector-like fermions. The black line is the upper limit on this cross-section from ATLAS [85] $8 \mathrm{TeV}$ and blue line is from CMS [84] $8 \mathrm{TeV}$ data in figure 12(a). Figure 12(b) shows the dijet limits from ATLAS(black) [88] $13 \mathrm{TeV}$ and CMS(blue) [89] $13 \mathrm{TeV}$ data. We can clearly say that the dijet limits are not strong enough to rule any of the models, if they have only one copy of constituent fermions.

In the figure 15, we have plotted (black solid line) the projected limit for $14 \mathrm{TeV}$ LHC at $3000 \mathrm{fb}^{-1}$ for the dijet cross section [90]. Assuming $Z_{B}^{\prime}$ model, $14 \mathrm{TeV}$ limits on mass of $Z_{B}^{\prime}$ and coupling between $Z_{B}^{\prime}$ gauge field with quark has been calculated in ref. [90]. Using this limit, we have calculated $14 \mathrm{TeV}$ projected limit on dijet cross-section. We have found that mass of vector-like fermion with colour representation six can be excluded up to $800-900 \mathrm{GeV}$ at the HL-LHC.

\subsubsection{Diphoton bounds}

The diphoton channel has played a very important role in discovering the Higgs Boson. It can be a very important channel to look at BSM physics. We present the production of diphoton channel as a function of the resonance mass considering one copy of constituent vector-like fermions in figure 13. Black line is the upper limit on this cross-section from ATLAS [82] $13 \mathrm{TeV}$ and blue line is from CMS [83] $13 \mathrm{TeV}$ data. It can be observed that the upper limits on cross-section can give stringent bound on the masses of vector-like fermions $\left(m_{\psi}=M / 2\right)$. 
There has been searches in $Z \gamma, Z Z$ and $W W$ resonances from these bound states. ATLAS [91] has performed a combination of individual searches in all-leptonic, and all hadronic final states to search for heavy bosons decaying to $Z Z$ and $W W$ with integrated luminosity of $20.3 \mathrm{fb}^{-1}$ at $8 \mathrm{TeV}$. The sensitivity is weaker than $\gamma \gamma$ channel for ATLAS [92] at $8 \mathrm{TeV}$ by around 1000. Both CMS [93] and ATLAS [94] have performed a resonance decaying to $Z \gamma$ at centre-of-mass energy of $8 \mathrm{TeV}$ at integrated luminosity 20.3 and $19.7 \mathrm{fb}^{-1}$ respectively. Where sensitivity is weaker than diphoton channel is weaker by order 10 .

CMS [95] has performed a searches in $Z \gamma$ resonance in leptonic channel final decay state at centre of mass energies of 8 and $13 \mathrm{TeV}$. The bounds are weaker than diphoton bounds by factor of 200 . ATLAS [96] has searched for heavy resonance decaying to $Z Z$ and $Z W$ pair decaying to leptonic and hadronic channels at a centre of mass energy $13 \mathrm{TeV}$ with total integrated luminosity $13.2 \mathrm{fb}^{-1}$. The sensitivity is still weaker by factor 1000 with respect to diphoton channel.

\section{Summary and outlook}

Unification of gauge couplings is one of the most important signatures of a successful Grand Unified Theory beyond the electroweak scale. We look for models with extra vector-like fermions at the weak scale which can lead to successful unification of gauge couplings. With two representation, we find a class of nine models leading to successful unification of gauge couplings. An interesting aspect of these is that all of them contain coloured vector-like fermion in the spectrum. The coloured set of the vector-like fermions can be probed at LHC by looking for bound states formed by them and their probable decays. We have already listed the present bounds from LHC for each successful model. The future runs of LHC are sensitive to further mass ranges of these particles. Finally, it would be interesting to look for complete GUT models with this particle spectrum.

\section{Acknowledgments}

The authors thank Tao Han, Rohini Godbole and Xerxes Tata for collaboration in the initial stages of the project. We thank Xerxes Tata for comments on the manuscript and a reference. SKV thanks visits to University of Pittsburgh and University of Hawaii where this project was started. SKV acknowledges support from IUSTFF Grant:JC-Physics Beyond Standard Model/23-2010 during the visit PITT PACC, Department of physics and Astronomy, University of Pittsburgh, U.S.A. and Department of Physics and Astronomy, University of Hawaii, Honolulu, U.S.A.. The work of B.B. is supported by the Department of Science and Technology, Government of India, under the Grant Agreement number IFA13-PH-75 (INSPIRE Faculty Award). P.B. thanks Palash B. Pal for giving a clue to calculate 6 dimensional representations of $\mathrm{SU}(3)$ generators. 


\section{A Two representation case}

Here we enlist the models which satisfy gauge coupling unification and positivity of higgs potenatial for Two fermion representation model, with $\Delta=3 \%$.

\begin{tabular}{|c|c|c||c|c||c|c|}
\hline $\begin{array}{c}\text { Mod } \\
\text { No. }\end{array}$ & Rep 1 & $\begin{array}{c}M_{\text {Rep1 }} \\
\text { GeV }\end{array}$ & Rep 2 & $\begin{array}{c}M_{\text {Rep2 }} \\
\text { GeV }\end{array}$ & $\begin{array}{c}M_{\text {GUT }} \\
\times 10^{16} \mathrm{GeV}\end{array}$ & $\alpha_{\text {GUT }}$ \\
\hline 1 & $1(1,1,1)$ & $(500-5000)$ & $1\left(3,2, \frac{1}{6}\right)$ & $(500-5000)$ & $\sim 0.15$ & $\sim 0.027$ \\
\hline 2 & $5\left(1,2, \frac{1}{2}\right)$ & $(250-500)$ & $1\left(6,1, \frac{1}{3}\right)$ & $(2500-5000)$ & $\sim 0.12$ & $\sim 0.035$ \\
\hline 3 & $3\left(1,2, \frac{1}{2}\right)$ & $(250-700)$ & $1(8,1,0)$ & $(1500-5000)$ & $\sim 0.14$ & $\sim 0.029$ \\
\hline 4 & $1(1,3,0)$ & $(500-5000)$ & $1\left(3,1, \frac{1}{3}\right)$ & $(500-5000)$ & $\sim 0.11$ & $\sim 0.025$ \\
\hline 5 & $1(1,3,0)$ & $(250-2200)$ & $2\left(3,1, \frac{1}{3}\right)$ & $(500-5000)$ & $\sim 0.13$ & $\sim 0.026$ \\
\hline 6 & $2(1,3,0)$ & $(1300-5000)$ & $3\left(3,1, \frac{1}{3}\right)$ & $(250-3000)$ & $\sim 0.67$ & $\sim 0.03$ \\
\hline 7 & $3(1,3,0)$ & $(3000-5000)$ & $1\left(6,2, \frac{5}{6}\right)$ & $(250-500)$ & $\sim 0.11$ & $\sim 0.32$ \\
\hline 8 & $1\left(3,1, \frac{2}{3}\right)$ & $(250-5000)$ & $1\left(3,2, \frac{1}{6}\right)$ & $(250-1100)$ & $\sim 0.15$ & $\sim 0.03$ \\
\hline
\end{tabular}

Table 5. Model with two vector-like fermions representation satisfying gauge coupling unification and vacuum stability condition, with $\Delta=3 \%$.

\section{B Three representation case}

Here we enlist the models which satisfy gauge coupling unification and positivity of higgs potenatial for three fermion representation model. Unlike Two Representation case, we made a restricted choice that all the representations and their copies are degenerate in mass of about $1 \mathrm{TeV}$, with up to ten copies in each representation. All of the models have unification scale less than $10^{16} \mathrm{GeV}$, which does not satisfy with Proton decay constraint. The models are listed below in table 6

\begin{tabular}{|c|c|c|c|c|c|}
\hline ModelNo. & Rep 1 & Rep 2 & Rep 3 & $\begin{array}{c}M_{\mathrm{GUT}} \\
\times 10^{16} \mathrm{GeV}\end{array}$ & $\alpha_{\mathrm{GUT}}$ \\
\hline 1 & $1(1,1,1)$ & $7\left(1,2, \frac{1}{2}\right)$ & $2(8,1,0)$ & 0.132 & 0.043 \\
2 & $7(1,1,1)$ & $5(1,3,0)$ & $3(8,1,0)$ & 0.414 & 0.082 \\
3 & $4(1,3,0)$ & $1\left(3,1, \frac{4}{3}\right)$ & $2(8,1,0)$ & 0.133 & 0.051 \\
4 & $8\left(1,2, \frac{1}{2}\right)$ & $1(1,3,0)$ & $9\left(3,1, \frac{1}{3}\right)$ & 0.209 & 0.077 \\
5 & $8\left(1,2, \frac{1}{2}\right)$ & $4\left(3,1, \frac{1}{3}\right)$ & $1(8,1,0)$ & 0.144 & 0.050 \\
\hline
\end{tabular}

Table 6. Models satisfying three fermion representation of gauge coupling unification and stable higgs potenatial with degenerate mass of $1 \mathrm{TeV}$. The representation is described as $n_{i}\left(R_{\mathrm{SU}(3)}, R_{\mathrm{SU}(2)}, R_{\mathrm{U}(1)}\right)$, where $n_{i}$ introduced earlier is the number of copies of the representation, $R_{G}$ is the representation of the field under the gauge group $\mathrm{G}$ of the SM. 


\section{Four representation case}

Here we enlist the models which satisfy gauge coupling unification and stable higgs potenatial upto grand unified scale for four fermion representation model. Here also we restricted representations and their copies are degenerate in mass of about $1 \mathrm{TeV}$. We have allowed for up to ten copies in each model. Except one model, all of the models have unification scale less than $10^{16} \mathrm{GeV}$, which does not satisfy with Proton decay constraint. The models are listed below in table 7

\section{Representations and Dynkin indices}

We considered all the $\mathrm{SU}(3) \times \mathrm{SU}(2) \times \mathrm{U}(1)$ representations coming from $\mathrm{SU}(5)$ representations upto dimension 75 . In table 8 , we listed those forty representations [41] with their contribution to beta function (i.e. Dynkin index) considering them as scalar fields. One can straight-forwardly derive corresponding Dynkin indices if the fileds are vector-like fermion just by multiplying the tabulated value with 2 if the representation is real and by multiplying with 4 if the considered representation is complex.

\section{E Mixing between SM particle with vector-like fermion}

In this section we will assume that the new vector-like fermions interact with the SM fermions via Yukawa interactions. SM contains $l=(1,2,-1 / 2) e_{R}=(1,1,-1), q=(3,2,1 / 6)$ and $d_{R}=(3,1,-1 / 3), u_{R}=(3,1,2 / 3)$ and Higgs doublet, $H=(1,2,1 / 2)$. It can be easily be understood that, among the vector-like fermions considered in this work, new vector-like fermions coupling to the SM ones with renormalisable couplings can only appear in top and bottom partner gauge-covariant multiplets, and in lepton and neutrino partner with definite $\mathrm{SU}(3)_{C} \times \mathrm{SU}(2)_{L} \times \mathrm{U}(1)_{Y}$ quantum numbers, which has been studied in [33, 34, 36, 37, 97100] and some of them tabulated in table 9 . Here we will briefly overview the leading order constraints coming from EW precision tests, direct searches at colliders and Higgs physics. It is reasonable to assume that, only third family of SM fermions have sizable contribution from new vector-like fermions.

\section{E.1 Vector like quarks}

Due to mixing of the SM top and bottom quark with vector-like fermions partners, the resulting physical up and down type quark mass eigenstates $u^{0}, c^{0}, t^{0}, T^{0}$ and $d^{0}, s^{0}, b^{0}, B^{0}$ may contain non-zero $T$ and $B$ components, leading to a deviation in their couplings to $\mathrm{Z}$ and $\mathrm{W} \pm$ bosons. In this case, the relation between weak and mass eigenstates for up quark can be parameterized as two $2 \times 2$ matrices $V_{L, R}^{U}$,

$$
\left(\begin{array}{c}
t_{L, R}^{0} \\
T_{L, R}^{0}
\end{array}\right)=\left(\begin{array}{cc}
\cos \theta_{L, R}^{u} & -\sin \theta_{L, R}^{u} \\
\sin \theta_{L, R}^{u} & \cos \theta_{L, R}^{u}
\end{array}\right)\left(\begin{array}{c}
t_{L, R} \\
T_{L, R}
\end{array}\right) .
$$




\begin{tabular}{|c|c|c|c|c|c|c|}
\hline ModelNo. & Rep 1 & Rep 2 & Rep 3 & Rep 4 & $\begin{array}{c}M_{\text {GUT }} \\
\times 10^{16} \mathrm{GeV} \\
\end{array}$ & $\alpha_{\mathrm{GUT}}$ \\
\hline 1 & $1(1,1,1)$ & $1\left(1,2, \frac{3}{2}\right)$ & $1\left(1,4, \frac{1}{2}\right)$ & $2\left(6,1, \frac{1}{3}\right)$ & 0.837 & 0.14 \\
\hline 2 & $1(1,1,1)$ & $4\left(1,2, \frac{1}{2}\right)$ & $1(1,3,0)$ & $1\left(6,1, \frac{1}{3}\right)$ & 0.112 & 0.038 \\
\hline 3 & $1(1,1,1)$ & $6(1,3,0)$ & $7\left(3,1, \frac{1}{3}\right)$ & $4\left(3,1, \frac{2}{3}\right)$ & 0.637 & 0.26 \\
\hline 4 & $1(1,1,1)$ & $7\left(1,2, \frac{1}{2}\right)$ & $2(1,3,0)$ & $10\left(3,1, \frac{1}{3}\right)$ & 0.317 & 0.11 \\
\hline 5 & $1(1,1,1)$ & $8\left(1,2, \frac{1}{2}\right)$ & $8\left(3,1, \frac{1}{3}\right)$ & $1\left(3,2, \frac{1}{6}\right)$ & 0.343 & 0.11 \\
\hline 6 & $2(1,1,1)$ & $3(1,3,0)$ & $1\left(3,2, \frac{5}{6}\right)$ & $2(8,1,0)$ & 0.193 & 0.063 \\
\hline 7 & $2(1,1,1)$ & $4(1,3,0)$ & $2\left(3,1, \frac{1}{3}\right)$ & $1\left(6,1, \frac{2}{3}\right)$ & 0.123 & 0.051 \\
\hline 8 & $2(1,1,1)$ & $4(1,3,0)$ & $2\left(3,1, \frac{2}{3}\right)$ & $1\left(6,1, \frac{1}{3}\right)$ & 0.154 & 0.051 \\
\hline 9 & $2(1,1,1)$ & $5\left(1,2, \frac{1}{2}\right)$ & $1\left(3,2, \frac{1}{6}\right)$ & $1\left(6,1, \frac{1}{3}\right)$ & 0.167 & 0.051 \\
\hline 10 & $2(1,1,1)$ & $5\left(1,2, \frac{1}{2}\right)$ & $1(1,3,0)$ & $2(8,1,0)$ & 0.137 & 0.044 \\
\hline 11 & $2(1,1,1)$ & $5\left(1,2, \frac{1}{2}\right)$ & $3(1,3,0)$ & $10\left(3,1, \frac{1}{3}\right)$ & 0.352 & 0.11 \\
\hline 12 & $2(1,1,1)$ & $6(1,3,0)$ & $8\left(3,1, \frac{1}{3}\right)$ & $3\left(3,1, \frac{2}{3}\right)$ & 0.763 & 0.28 \\
\hline 13 & $3(1,1,1)$ & $5(1,3,0)$ & $3\left(3,1, \frac{2}{3}\right)$ & $2(8,1,0)$ & 0.274 & 0.080 \\
\hline 14 & $3(1,1,1)$ & $6\left(1,2, \frac{1}{2}\right)$ & $1\left(3,2, \frac{1}{6}\right)$ & $2(8,1,0)$ & 0.236 & 0.062 \\
\hline 15 & $4(1,1,1)$ & $2(1,3,0)$ & $2\left(3,2, \frac{1}{6}\right)$ & $1\left(6,1, \frac{2}{3}\right)$ & 0.269 & 0.082 \\
\hline 16 & $4(1,1,1)$ & $4\left(1,2, \frac{1}{2}\right)$ & $2\left(3,2, \frac{1}{6}\right)$ & $1\left(6,1, \frac{1}{3}\right)$ & 0.358 & 0.082 \\
\hline 17 & $5(1,1,1)$ & $1\left(1,2, \frac{1}{2}\right)$ & $1\left(1,4, \frac{1}{2}\right)$ & $2\left(6,1, \frac{1}{3}\right)$ & 1.09 & 0.15 \\
\hline 18 & $5(1,1,1)$ & $5\left(1,2, \frac{1}{2}\right)$ & $2\left(3,2, \frac{1}{6}\right)$ & $2(8,1,0)$ & 0.721 & 0.13 \\
\hline 19 & $5(1,1,1)$ & $5(1,3,0)$ & $4\left(3,1, \frac{1}{3}\right)$ & $1\left(6,1, \frac{1}{3}\right)$ & 0.300 & 0.081 \\
\hline 20 & $1(1,1,1)$ & $1(1,3,1)$ & $2\left(3,2, \frac{1}{6}\right)$ & $1\left(6,1, \frac{2}{3}\right)$ & 0.207 & 0.081 \\
\hline 21 & $1(1,1,2)$ & $4\left(1,2, \frac{1}{2}\right)$ & $2\left(3,2, \frac{1}{6}\right)$ & $1\left(6,1, \frac{1}{3}\right)$ & 0.276 & 0.081 \\
\hline 22 & $1(1,1,2)$ & $4(1,3,0)$ & $2\left(3,1, \frac{1}{3}\right)$ & $1\left(6,1, \frac{1}{3}\right)$ & 0.157 & 0.051 \\
\hline 23 & $1(1,1,2)$ & $6(1,3,0)$ & $10\left(3,1, \frac{1}{3}\right)$ & $1\left(3,1, \frac{2}{3}\right)$ & 0.748 & 0.27 \\
\hline 24 & $1\left(1,2, \frac{1}{2}\right)$ & $2(1,3,0)$ & $1\left(3,2, \frac{5}{6}\right)$ & $1\left(6,1, \frac{1}{3}\right)$ & 0.130 & 0.051 \\
\hline 25 & $3\left(1,2, \frac{1}{2}\right)$ & $1\left(1,2, \frac{3}{2}\right)$ & $2\left(3,2, \frac{1}{6}\right)$ & $1\left(6,1, \frac{1}{3}\right)$ & 0.266 & 0.081 \\
\hline 26 & $3\left(1,2, \frac{1}{2}\right)$ & $4(1,3,0)$ & $7\left(3,1, \frac{1}{3}\right)$ & $3\left(3,1, \frac{2}{3}\right)$ & 0.280 & 0.11 \\
\hline 27 & $4\left(1,2, \frac{1}{2}\right)$ & $1(1,3,1)$ & $1\left(3,1, \frac{1}{3}\right)$ & $2(8,1,0)$ & 0.142 & 0.051 \\
\hline 28 & $5\left(1,2, \frac{1}{2}\right)$ & $1(1,3,0)$ & $1\left(3,1, \frac{2}{3}\right)$ & $1\left(6,1, \frac{1}{3}\right)$ & 0.112 & 0.043 \\
\hline 29 & $5\left(1,2, \frac{1}{2}\right)$ & $1(1,3,1)$ & $9\left(3,1, \frac{1}{3}\right)$ & $1\left(3,2, \frac{1}{6}\right)$ & 0.836 & 0.28 \\
\hline 30 & $5\left(1,2, \frac{1}{2}\right)$ & $3(1,3,0)$ & $8\left(3,1, \frac{1}{3}\right)$ & $2\left(3,1, \frac{2}{3}\right)$ & 0.269 & 0.11 \\
\hline 31 & $6\left(1,2, \frac{1}{2}\right)$ & $2(1,3,0)$ & $8\left(3,1, \frac{1}{3}\right)$ & $1\left(3,1, \frac{2}{3}\right)$ & 0.200 & 0.077 \\
\hline 32 & $6\left(1,2, \frac{1}{2}\right)$ & $4\left(3,1, \frac{1}{3}\right)$ & $3\left(3,1, \frac{2}{3}\right)$ & $2\left(3,2, \frac{1}{6}\right)$ & 0.922 & 0.30 \\
\hline 33 & $8\left(1,2, \frac{1}{2}\right)$ & $7\left(3,1, \frac{1}{3}\right)$ & $1\left(3,1, \frac{2}{3}\right)$ & $1\left(3,2, \frac{1}{6}\right)$ & 0.319 & 0.11 \\
\hline 34 & $1\left(1,2, \frac{3}{2}\right)$ & $1(1,3,1)$ & $2\left(3,2, \frac{1}{6}\right.$ & $2(8,1,0)$ & 0.570 & 0.13 \\
\hline 35 & $1(1,3,0)$ & $2(1,3,1)$ & $3\left(3,1, \frac{1}{3}\right)$ & $2(8,1,0)$ & 0.239 & 0.080 \\
\hline 36 & $1\left(1,2, \frac{3}{2}\right)$ & $4(1,3,0)$ & $3\left(3,1, \frac{1}{3}\right)$ & $1\left(6,1, \frac{1}{3}\right)$ & 0.185 & 0.062 \\
\hline 37 & $3(1,3,0)$ & $1\left(3,1, \frac{2}{3}\right)$ & $1\left(3,2, \frac{5}{6}\right)$ & $1\left(6,1, \frac{1}{3}\right)$ & 0.156 & 0.062 \\
\hline 38 & $3(1,3,0)$ & $1\left(3,1, \frac{4}{3}\right)$ & $1\left(3,2, \frac{1}{6}\right)$ & $1\left(6,1, \frac{1}{3}\right)$ & 0.157 & 0.062 \\
\hline 39 & $4(1,3,0)$ & $1(1,3,1)$ & $9\left(3,1, \frac{1}{3}\right)$ & $2\left(3,1, \frac{2}{3}\right)$ & 0.681 & 0.27 \\
\hline 40 & $5(1,3,0)$ & $1\left(3,1, \frac{1}{3}\right)$ & $5\left(3,1, \frac{2}{3}\right)$ & $1(8,1,0)$ & 0.223 & 0.079 \\
\hline 41 & $5(1,3,0)$ & $5\left(3,1, \frac{1}{3}\right)$ & $1\left(3,1, \frac{4}{3}\right)$ & $1(8,1,0)$ & 0.188 & 0.078 \\
\hline
\end{tabular}

Table 7. Models satisfying four fermion representation of gauge coupling unification and stable higgs potenatial with degenerate mass of $1 \mathrm{TeV}$. The representation is described as $n_{i}\left(R_{\mathrm{SU}(3)}, R_{\mathrm{SU}(2)}, R_{\mathrm{U}(1)}\right)$, where $n_{i}$ introduced earlier is the number of copies of the representation, $R_{G}$ is the representation of the field under the gauge group G of the SM. 


\begin{tabular}{|c|c|c|c|c|c|c|c|}
\hline S.No. & SM Rep & Source & Dynkin Indices & S.No. & SM Rep & Source & Dynkin Indices \\
\hline 1 & $(1,1,1)$ & 10 & $\left(0,0,-\frac{1}{5}\right)$ & 21 & $\left(3,2, \frac{7}{6}\right)$ & $\overline{45}, \overline{50}$ & $\left(-\frac{1}{3},-\frac{1}{2},-\frac{49}{30}\right)$ \\
\hline 2 & $(1,1,2)$ & $\overline{50}$ & $\left(0,0,-\frac{4}{5}\right)$ & 22 & $\left(3,3,-\frac{1}{3}\right)$ & 45,70 & $\left(-\frac{1}{2},-2,-\frac{1}{5}\right)$ \\
\hline 3 & $(1,1,3)$ & & $\left(0,0,-\frac{9}{5}\right)$ & 23 & $\left(3,3, \frac{2}{3}\right)$ & $\overline{35}, \overline{40}$ & $\left(-\frac{1}{2},-2,-\frac{4}{5}\right)$ \\
\hline 4 & $(1,1,4)$ & & $\left(0,0,-\frac{16}{5}\right)$ & 24 & $\left(\overline{3}, 3, \frac{4}{3}\right)$ & 70 & $\left(-\frac{1}{2},-2,-\frac{16}{5}\right)$ \\
\hline 5 & $(1,1,5)$ & & $(0,0,-5)$ & 25 & $\left(3,4, \frac{7}{6}\right)$ & $\overline{70^{\prime}}$ & $\left(-\frac{2}{3},-5,-\frac{49}{15}\right)$ \\
\hline 6 & $\left(1,2, \frac{1}{2}\right)$ & $5,45,70$ & $\left(0,-\frac{1}{6},-\frac{1}{10}\right)$ & 26 & $\left(6,1, \frac{1}{3}\right)$ & $\overline{45}$ & $\left(-\frac{5}{6}, 0,-\frac{2}{15}\right)$ \\
\hline 7 & $\left(1,2,-\frac{3}{2}\right)$ & 40 & $\left(0,-\frac{1}{6},-\frac{9}{10}\right)$ & 27 & $\left(6,1,-\frac{2}{3}\right)$ & 15 & $\left(-\frac{5}{6}, 0,-\frac{8}{15}\right)$ \\
\hline 8 & $(1,3,0)$ & 24 & $\left(0,-\frac{2}{3}, 0\right)$ & 28 & $\left(6,1, \frac{4}{3}\right)$ & 50 & $\left(-\frac{5}{6}, 0,-\frac{32}{15}\right)$ \\
\hline 9 & $(1,3,1)$ & 15 & $\left(0,-\frac{2}{3},-\frac{3}{5}\right)$ & 29 & $\left(\overline{6}, 2, \frac{1}{6}\right)$ & 35,40 & $\left(-\frac{5}{3},-1,-\frac{1}{15}\right)$ \\
\hline 10 & $\left(1,4, \frac{1}{2}\right)$ & 70 & $\left(0,-\frac{5}{3},-\frac{1}{5}\right)$ & 30 & $\left(6,2, \frac{5}{6}\right)$ & 75 & $\left(-\frac{5}{3},-1,-\frac{5}{3}\right)$ \\
\hline 11 & $\left(1,4,-\frac{3}{2}\right)$ & 35 & $\left(0,-\frac{5}{3},-\frac{9}{5}\right)$ & 31 & $\left(6,2,-\frac{7}{6}\right)$ & 70 & $\left(-\frac{5}{3},-1,-\frac{49}{15}\right)$ \\
\hline 12 & $(1,5,-2)$ & $70^{\prime}$ & $\left(0,-\frac{10}{3},-4\right)$ & 32 & $\left(6,3, \frac{1}{3}\right)$ & $\overline{50}, \overline{70}$ & $\left(-\frac{5}{2},-4,-\frac{2}{5}\right)$ \\
\hline 13 & $(1,5,1)$ & & $\left(0,-\frac{10}{3},-1\right)$ & 33 & $(8,1,0)$ & 24 & $(-1,0,0)$ \\
\hline 14 & $(1,5,0)$ & & $\left(0,-\frac{10}{3}, 0\right)$ & 34 & $(8,1,1)$ & 40 & $\left(-1,0,-\frac{8}{5}\right)$ \\
\hline 15 & $\left(3,1,-\frac{1}{3}\right)$ & $5,45,50,70$ & $\left(-\frac{1}{6}, 0,-\frac{1}{15}\right)$ & 35 & $\left(8,2, \frac{1}{2}\right)$ & $45,50,70$ & $\left(-2,-\frac{4}{3},-\frac{4}{5}\right)$ \\
\hline 16 & $\left(\overline{3}, 1,-\frac{2}{3}\right)$ & 10,40 & $\left(-\frac{1}{6}, 0,-\frac{4}{15}\right)$ & 36 & $(8,3,0)$ & 75 & $\left(-3,-\frac{16}{3}, 0\right)$ \\
\hline 17 & $\left(\overline{3}, 1, \frac{4}{3}\right)$ & 45 & $\left(-\frac{1}{6}, 0,-\frac{16}{15}\right)$ & 37 & $(\overline{10}, 1,1)$ & 35 & $\left(-\frac{5}{2}, 0,-2\right)$ \\
\hline 18 & $\left(3,1, \frac{5}{3}\right)$ & 75 & $\left(-\frac{1}{6}, 0,-\frac{5}{3}\right)$ & 38 & $\left(\overline{10}, 2, \frac{1}{2}\right)$ & $70^{\prime}$ & $\left(-5,-\frac{5}{3},-1\right)$ \\
\hline 19 & $\left(3,2, \frac{1}{6}\right)$ & $10,15,40$ & $\left(-\frac{1}{3},-\frac{1}{2},-\frac{1}{30}\right)$ & 39 & $\left(15,1,-\frac{1}{3}\right)$ & 70 & $\left(-\frac{10}{3}, 0,-\frac{1}{3}\right)$ \\
\hline 20 & $\left(3,2,-\frac{5}{6}\right)$ & 24,75 & $\left(-\frac{1}{3},-\frac{1}{2},-\frac{5}{6}\right)$ & 40 & $\left(15,1, \frac{4}{3}\right)$ & $70^{\prime}$ & $\left(-\frac{10}{3}, 0,-\frac{16}{3}\right)$ \\
\hline
\end{tabular}

Table 8. Representation of fields considered in this paper. In the column entitled with "SM Rep" we put incomplete multiplets of SU(5) and the entries inside the brackets are $\mathrm{SU}(3), \mathrm{SU}(2)$ and U(1) representations respectively. In the column with title we'd written the $\mathrm{SU}(5)$ representations from which those representations are coming. Dynkin indices are calculated assuming the fields are scalar fields. Note that we had considered up the SU(5) representation of dimension 75 . There are some extra representations as well.

Similar unitary matrices can be written for down sector. The mixing angles in the left and right sectors are not independent, but have a relation (see also [101-103])

$$
\begin{aligned}
\tan \theta_{R}^{q} & =\frac{m_{q}}{m_{Q}} \tan \theta_{L}^{q} \quad \text { (singlets, triplets), } \\
\tan \theta_{L}^{q} & =\frac{m_{q}}{m_{Q}} \tan \theta_{R}^{q} \quad \text { (doublets), }
\end{aligned}
$$

where $m_{q}$ and $m_{Q}$ are the mass of SM fermion and vector-like fermion respectively.

This mixing gives new contributions to the oblique parameters S and T [104], which is precisely measured at LEP and SLC. The contributions to S, T in models with T, B singlets and (T B) doublets are studied in [34, 37, 38, 105], which would give a constraints in mixing parameters between SM and their vector-like fermions partners. For singlet B quark, the constraints from $R_{b}$ is strong, which gives upper bound on mixing $\sin \theta_{L}^{d}$ to be 


\begin{tabular}{|c|c|}
\hline Vector-Like Fermion & Couples to \\
\hline$E(1,1,-1)$ & $l, e_{R}$ \\
\hline$L\left(1,2,-\frac{1}{2}\right)$ & $l, e_{R}$ \\
\hline$\Lambda\left(1,2,-\frac{3}{2}\right)$ & $e_{R}$ \\
\hline$\Delta(1,3,-1)$ & $l$ \\
\hline$\Sigma(1,3,0)$ & $l$ \\
\hline$T\left(3,1,+\frac{2}{3}\right)$ & $q, u_{R}$ \\
\hline$B\left(3,1,-\frac{1}{3}\right)$ & $q, d_{R}$ \\
\hline$X_{T}\left(3,2,+\frac{7}{6}\right)$ & $u_{R}$ \\
\hline$Q\left(3,2,+\frac{1}{6}\right)$ & $q, d_{R}, u_{R}$ \\
\hline$Y_{B}\left(3,2,-\frac{5}{6}\right)$ & $d_{R}$ \\
\hline$X_{Q}\left(3,3,+\frac{2}{3}\right)$ & $q$ \\
\hline$Y_{Q}\left(3,3,-\frac{1}{3}\right)$ & $q$ \\
\hline
\end{tabular}

Table 9. Vector-like fermions, that provide a consistent extension of the SM and modify the Higgs boson couplings [37].

0.04. For singlet $\mathrm{T}$ quark upper bound of $\sin \theta_{L}^{u}$ is 0.15 to 0.10 for mass range $600 \mathrm{GeV}$ to $2 \mathrm{TeV}$ respectively, from $\mathrm{S}$ and $\mathrm{T}$ parameter. For $(\mathrm{T} \mathrm{B})$ doublet, the constraints from EW precision gives upper bound on $\sin \theta_{R}^{d}$ to be 0.06 and, $\sin \theta_{R}^{u}$ between 0.13 to 0.09 for mass range $600 \mathrm{GeV}$ to $2 \mathrm{TeV}$ respectively, considering the splitting between $M_{B}$ and $M_{T}$ of $2 \mathrm{GeV}$.

Direct searches. A full model of vector-like Quark decaying to SM particles and search strategies to discover at LHC has been studied in refs. [34, 106, 107] and ref. within. The singlet $T$ Quark decays as,

$$
T \rightarrow W^{+} b, \quad T \rightarrow Z t, \quad T \rightarrow H t .
$$

The singlet $B$ quark decays are

$$
B \rightarrow W^{-} t, \quad B \rightarrow Z b, \quad B \rightarrow H b .
$$

$T B$ doublet assuming that they couple to the third generation, are the same as for singlets,

$$
\begin{array}{lll}
T \rightarrow W^{+} b, & T \rightarrow Z t, & T \rightarrow H t, \\
T \rightarrow W^{-} t, & B \rightarrow Z b, & T \rightarrow H b .
\end{array}
$$

We would summaries the mass constraints coming from direct searches of VLQ at the LHC.

For Integrated luminosity of $19.5 \mathrm{fb}^{-1}$ at $\sqrt{s}=8 \mathrm{TeV}$ CMS [108] experiment at the Large Hadron Collider searched for the $T$ quark decaying into three different final states, $\mathrm{bW}$, tZ, and tH. The search is carried out using events with at least one isolated lepton. 
The lower limits are set on the T quark mass at $95 \%$ confidence level between 687 and $782 \mathrm{GeV}$ for all possible values of the branching fractions into the three different final states assuming strong production.

A search in CMS [109] is performed in five exclusive channels: a single- lepton channel, a multilepton channel, two all-hadronic channels optimized either for the bW or the tH decay, and one channel in which the Higgs boson decays into two photons. A statistical combination of these results is performed and lower limits on the $\mathrm{T}$ quark mass are set. Depending on the branch- ing fractions, lower mass limits between 720 and $920 \mathrm{GeV}$ at 95 $\%$ confidence level are found. A search similar to Top like vector quark, heavy B quark vec- tor couplings to $\mathrm{W}, \mathrm{Z}$, and $\mathrm{H}$ bosons, is carried out by CMS experiment [110]. The B quark is assumed to be pair produced and to decay in one of three ways: to tW, bZ, or bH. The search is carried out in final states with one, two, and more than two charged leptons, as well as in fully hadronic final states.Each of the channels in the exclusive final-state topologies is designed to be sensitive to specific combinations of the B quark-antiquark pair decays. A statistical combination of these results gives lower limits on the B quark mass between $740 \mathrm{GeV}$ and $900 \mathrm{GeV}$ with $95 \%$ confidence level, depending on the values of the branching fractions of the $\mathrm{B}$ quark to $\mathrm{tW}, \mathrm{bZ}$, and $\mathrm{bH}$.

ATLAS has also searched for exotic quark, heavy X quark with $Q=5 / 3$ decaying to tW gives a lower bound of mass $840 \mathrm{GeV}$ [111] with $95 \%$ C.L. Quark Y with chagre $Q=-4 / 3$ decaying to $\mathrm{Wb}$ gives lower bound of mass $770 \mathrm{GeV}[112]$ with $95 \%$ C.L. The experimental searches assume pair production via strong interactions and prominent decays in the indicated channels.

\section{E.2 Vector like leptons}

In this section we discuss new colourless fermions. Weak iso-triplet with zero hyper-charge vector-like fermion can couple to left $l$ handed SM fermions and higgs as:

$$
\mathcal{L}_{\Sigma}=-\sqrt{2} Y_{\Sigma} \bar{\Sigma} l \tilde{H}-\frac{1}{2} \operatorname{Tr}\left(\bar{\Sigma} M_{\Sigma} \Sigma^{c}\right)+\text { h.c. }
$$

where the matrix notation of $\Sigma$ is as follows

$$
\Sigma_{\equiv} \sqrt{2} \Sigma^{a} \tau^{a}=\left(\begin{array}{cc}
\frac{1}{\sqrt{2}} \Sigma^{0} & -\Sigma^{+} \\
\Sigma^{-} & -\frac{1}{\sqrt{2}} \Sigma^{0}
\end{array}\right)
$$

The contribution of $\Sigma$ to the EW precision parameters is vanishingly small [33], since the mixing angle are suppressed by $\sim m_{\nu} / M_{\Sigma}$ and the loop induced mass splitting between the $M_{\Sigma^{ \pm}}-M_{\Sigma^{0}}=164-165 \mathrm{GeV}$ [113]. In the limit $Y_{\Sigma} \ll M_{\Sigma} / v$ we can realize it as a type III seesaw model [67] with neutrino mass $m_{\nu}=Y_{\Sigma}^{2} v^{2} / M_{\Sigma}$.

In the limit $Y_{\Sigma} \rightarrow 0$, this can be realized as a wino like dark matter [70].

SM fermions can also couple to four different possible vector-like leptons, a weak singlet $\mathrm{E}$, a weak doublet $\mathrm{L}$ or $\Lambda$, a weak triplet $\Delta$. The effect of these vector-like leptons on modification on the Higgs decays, anomalous magnetic moment to the muon and lepton flavour violation decays are studied in refs. [35, 100, 114-117]. 
Direct search. The limits on $M$ strongly depend on the SM generation that couples to the heavy leptons. The limits on doublet L, couplings only to the third generation is $M_{L}$ $>270 \mathrm{GeV}$ and coupling with $e$ and $\mu$ gives bound of $M_{L}>450 \mathrm{GeV}$, ref. [114], while the LEP limit remains more constraining in the case of the singlet E, $M_{E}>100 \mathrm{GeV}$. For the exotic doublet $\Lambda$ with a doubly-charged component, ref. [115] reports $M_{\Lambda}>320 \mathrm{GeV}$.

\section{F Earlier scan of models by Tom Rizzo}

In this section we update the work done in ref. [13]. They studied the grand unified theories in context of additional degree of freedom at electroweak scale. S (F) indicates that the quantum numbers following it refer to a complex scalar (vector like fermion) representation. $N_{A}\left(N_{B}\right)$ is the number of fields of type A (B) in the scenario.

\begin{tabular}{|c|c|c|c|c|c|c|c|c|c|c|c|c|}
\hline$N_{A}$ & & $\mathrm{SU}(3)$ & $\mathrm{SU}(2)$ & $\mathrm{U}(1)$ & $N_{B}$ & $\mathrm{SU}(3)$ & $\mathrm{SU}(2)$ & $\mathrm{U}(1)$ & $M_{\mathrm{GUT}}$ & $\alpha_{\mathrm{GUT}}$ & Status \\
\hline 1 & $S$ & 8 & 1 & $\frac{2}{3}$ & 1 & $S$ & 3 & 3 & 1 & $5.15393 \times 10^{14}$ & 0.0310221 & No \\
\hline 2 & $S$ & 3 & 2 & $\frac{1}{6}$ & 2 & $S$ & 1 & 2 & $\frac{1}{2}$ & $5.07162 \times 10^{14}$ & 0.026024 & Yes \\
\hline 2 & $S$ & 6 & 2 & $\frac{1}{2}$ & 2 & $S$ & 1 & 3 & $\frac{2}{3}$ & $4.07143 \times 10^{16}$ & 0.0353412 & Yes \\
\hline 2 & $S$ & 6 & 2 & $\frac{1}{6}$ & 2 & $S$ & 1 & 3 & 0 & $8.54256 \times 10^{20}$ & 0.0326849 & No \\
\hline 1 & $F$ & 3 & 2 & $\frac{1}{6}$ & 1 & $F$ & 1 & 1 & 1 & $5.07162 \times 10^{14}$ & 0.0283188 & Yes \\
\hline 1 & $F$ & 3 & 2 & $\frac{1}{2}$ & 1 & $S$ & 8 & 2 & $\frac{1}{6}$ & $1.29764 \times 10^{17}$ & 0.034587 & Yes \\
\hline 1 & $F$ & 3 & 2 & $\frac{1}{6}$ & 1 & $S$ & 1 & 1 & 2 & $5.07162 \times 10^{14}$ & 0.0283188 & Yes \\
\hline 1 & $F$ & 3 & 2 & $\frac{1}{6}$ & 2 & $S$ & 3 & 1 & 1 & $1.69262 \times 10^{15}$ & 0.0292299 & Yes \\
\hline 1 & $F$ & 3 & 1 & 0 & 2 & $S$ & 1 & 3 & $\frac{2}{3}$ & $5.02121 \times 10^{14}$ & 0.0264338 & Yes \\
\hline 1 & $F$ & 3 & 1 & $\frac{1}{3}$ & 2 & $S$ & 1 & 3 & 1 & $1.7518 \times 10^{14}$ & 0.0275409 & Yes \\
\hline 1 & $S$ & 8 & 1 & $\frac{2}{3}$ & 1 & $S$ & 3 & 1 & $\frac{5}{3}$ & $1.91539 \times 10^{22}$ & 0.0440168 & No \\
\hline 1 & $S$ & 8 & 2 & $\frac{1}{6}$ & 1 & $S$ & 1 & 3 & 1 & $2.10093 \times 10^{16}$ & 0.0285893 & Yes \\
\hline 1 & $F$ & 3 & 2 & $\frac{1}{6}$ & 1 & $F$ & 3 & 1 & 0 & $4.07855 \times 10^{16}$ & 0.0276959 & Yes \\
\hline 1 & $F$ & 1 & 2 & $\frac{1}{6}$ & 1 & $F$ & 8 & 2 & $\frac{1}{6}$ & $8.51879 \times 10^{48}$ & -0.0774188 & No \\
\hline 1 & $F$ & 3 & 2 & $\frac{1}{6}$ & 2 & $S$ & 3 & 1 & $\frac{2}{3}$ & $1.90667 \times 10^{15}$ & 0.0281254 & Yes \\
\hline 2 & $F$ & 1 & 2 & $\frac{1}{2}$ & 1 & $S$ & 8 & 2 & $\frac{1}{2}$ & $5.07162 \times 10^{14}$ & 0.0310574 & Yes \\
\hline 2 & $F$ & 1 & 2 & $\frac{1}{6}$ & 1 & $S$ & 6 & 1 & 0 & $1.22375 \times 10^{15}$ & 0.0258567 & Yes \\
\hline 2 & $F$ & 1 & 2 & $\frac{1}{6}$ & 1 & $S$ & 6 & 1 & $\frac{1}{3}$ & $6.47456 \times 10^{14}$ & 0.0259887 & Yes \\
\hline 2 & $F$ & 1 & 1 & 0 & 1 & $S$ & 3 & 3 & $\frac{2}{3}$ & $3.63426 \times 10^{14}$ & 0.0272945 & Yes \\
\hline 2 & $F$ & 3 & 1 & $\frac{2}{3}$ & 1 & $S$ & 6 & 3 & 1 & $1.2987 \times 10^{16}$ & 0.0690751 & No \\
\hline 2 & $F$ & 3 & 2 & $\frac{1}{2}$ & 1 & $S$ & 8 & 1 & 0 & $8.1903 \times 10^{15}$ & 0.0390761 & Yes \\
\hline 2 & $F$ & 3 & 2 & $\frac{1}{2}$ & 1 & $S$ & 8 & 1 & $\frac{1}{3}$ & $3.12815 \times 10^{15}$ & 0.0388947 & Yes \\
\hline 2 & $F$ & 1 & 2 & $\frac{1}{6}$ & 2 & $S$ & 6 & 2 & $\frac{1}{2}$ & $1.29764 \times 10^{17}$ & 0.034587 & Yes \\
\hline 2 & $F$ & 3 & 2 & $\frac{1}{6}$ & 2 & $S$ & 6 & 2 & $\frac{5}{6}$ & $6.99517 \times 10^{17}$ & 0.0713552 & Yes \\
\hline
\end{tabular}




\section{G Two loop beta function}

For Standard Model, in Yukawa sector the beta function are [44-46]

$$
\frac{d Y_{u, d, e}}{d t}=Y_{u, d, e} \frac{1}{16 \pi^{2}} \beta_{u, d, e}^{(1)}+\frac{1}{\left(16 \pi^{2}\right)^{2}} \beta_{u, d, e}^{(2)}
$$

where one loop contribution are given as

$$
\begin{aligned}
& \beta_{u}^{(1)}=\frac{3}{2}\left(Y_{u}^{\dagger} Y_{u}-Y_{d}^{\dagger} Y_{d}\right)+Y_{2}(S)-\left(\frac{17}{20} g_{1}^{2}+\frac{9}{4} g_{2}^{2}+8 g_{3}^{2}\right) \\
& \beta_{d}^{(1)}=\frac{3}{2}\left(Y_{d}^{\dagger} Y_{d}-Y_{u}^{\dagger} Y_{u}\right)+Y_{2}(S)-\left(\frac{1}{4} g_{1}^{2}+\frac{9}{4} g_{2}^{2}+8 g_{3}^{2}\right) \\
& \beta_{e}^{(1)}=\frac{3}{2} Y_{e}^{\dagger} Y_{e}+Y_{2}(S)-\frac{9}{4}\left(g_{1}^{2}+g_{2}^{2}\right)
\end{aligned}
$$

with

$$
Y_{2}(S)=\operatorname{Tr}\left(3 Y_{u}^{\dagger} Y_{u}+3 Y_{d}^{\dagger} Y_{d}+Y_{e}^{\dagger} Y_{e}\right)
$$

the two-loop contribution are given as

$$
\begin{aligned}
\beta_{u}^{(2)}= & \frac{3}{2}\left(Y_{u}^{\dagger} Y_{u}\right)^{2}-Y_{u}^{\dagger} Y_{u} Y_{d}^{\dagger} Y_{d}-\frac{1}{4} Y_{d}^{\dagger} Y_{d} Y_{u}^{\dagger} Y_{u}+\frac{11}{4}\left(Y_{d}^{\dagger} Y_{d}\right)^{2}+Y_{2}(S)\left(\frac{5}{4} Y_{d}^{\dagger} Y_{d}-\frac{9}{4} Y_{u}^{\dagger} Y_{u}\right) \\
& -\chi_{4}(S)+\frac{3}{2} \lambda^{2}-6 \lambda Y_{u}^{\dagger} Y_{u}+\left(\frac{223}{80} g_{1}^{2}+\frac{135}{16} g_{2}^{2}+16 g_{3}^{2}\right) Y_{u}^{\dagger} Y_{u} \\
& -\left(\frac{43}{80} g_{1}^{2}-\frac{9}{16} g_{2}^{2}+16 g_{3}^{2}\right) Y_{d}^{\dagger} Y_{d}+\frac{5}{2} Y_{4}(S)+\left(\frac{9}{200}+\frac{29}{45} n_{g}\right) g_{1}^{4} \\
& -\frac{9}{20} g_{1}^{2} g_{2}^{2}+\frac{19}{15} g_{1}^{2} g_{3}^{2}-\left(\frac{35}{4}-n_{g}\right) g_{2}^{4}+9 g_{2}^{2} g_{3}^{2}-\left(\frac{404}{3}-\frac{80}{9} n_{g}\right) g_{3}^{4} \\
\beta_{d}^{(2)}= & \frac{3}{2}\left(Y_{d}^{\dagger} Y_{d}\right)^{2}-Y_{d}^{\dagger} Y_{d} Y_{u}^{\dagger} Y_{u}-\frac{1}{4} Y_{u}^{\dagger} Y_{u} Y_{d}^{\dagger} Y_{d}+\frac{11}{4}\left(Y_{u}^{\dagger} Y_{u}\right)^{2}+Y_{2}(S)\left(\frac{5}{4} Y_{u}^{\dagger} Y_{u}-\frac{9}{4} Y_{d}^{\dagger} Y_{d}\right) \\
& -\chi_{4}(S)+\frac{3}{2} \lambda^{2}-2 \lambda 3 Y_{d}^{\dagger} Y_{d}+\left(\frac{187}{80} g_{1}^{2}+\frac{135}{16} g_{2}^{2}+16 g_{3}^{2}\right) Y_{d}^{\dagger} Y_{d} \\
& -\left(\frac{79}{80} g_{1}^{2}-\frac{9}{16} g_{2}^{2}+16 g_{3}^{2}\right) Y_{u}^{\dagger} Y_{u}+\frac{5}{2} Y_{4}(S)-\left(\frac{29}{200}+\frac{1}{45} n_{g}\right) g_{1}^{4} \\
& -\frac{27}{20} g_{1}^{2} g_{2}^{2}+\frac{31}{15} g_{1}^{2} g_{3}^{2}-\left(\frac{35}{4}-n_{g}\right) g_{2}^{4} 9 g_{2}^{2} g_{3}^{2}-\left(\frac{404}{3}-\frac{80}{9} n_{g}\right) g_{3}^{4} \\
\beta_{e}^{(2)}= & \frac{3}{2}\left(Y_{e}^{\dagger} Y_{e}\right)^{2}-\frac{9}{4} Y_{2}(S) Y_{e}^{\dagger} Y_{e}-\chi_{4}(S)+\frac{3}{2} \lambda^{2}-6 \lambda Y_{e}^{\dagger} Y_{e}+\left(\frac{387}{80} g_{1}^{2}+\frac{135}{15} g_{2}^{2}\right) Y_{e}^{\dagger} Y_{e} \\
& \left.+\frac{5}{2} Y_{4}(S)+\left(\frac{51}{200}+\frac{11}{5} n_{g}\right) g_{1}^{4}+\frac{27}{20} g_{1}^{2} g_{2}^{2}-\left(\frac{35}{4}-n_{g}\right) g_{2}^{4}\right)
\end{aligned}
$$

with

$$
Y_{4}(S)=\left(\frac{17}{20} g_{1}^{2}+\frac{9}{4} g_{2}^{2}+8 g_{3}^{2}\right) \operatorname{Tr}\left[Y_{u}^{\dagger} Y_{u}\right]+\left(\frac{1}{4} g_{1}^{2}+\frac{9}{4} g_{2}^{2}+8 g_{3}^{2}\right) \operatorname{Tr}\left[Y_{d}^{\dagger} Y_{d}\right]+\frac{3}{4}\left(g_{1}^{2}+g_{2}^{2}\right) \operatorname{Tr}\left[Y_{e}^{\dagger} Y_{e}\right]
$$


and

$$
\chi_{4}(S)=\frac{9}{4}\left(3\left(Y_{u}^{\dagger} Y_{u}\right)^{2}+3\left(Y_{d}^{\dagger} Y_{d}\right)^{2}+\left(Y_{e}^{\dagger} Y_{e}\right)^{2}-\frac{2}{3} Y_{u}^{\dagger} Y_{u} Y_{d}^{\dagger} Y_{d}\right)
$$

In Higgs sector we present $\beta$ functions for the quartic coupling:

$$
\frac{d \lambda}{d t}=\frac{1}{16 \pi^{2}} \beta_{\lambda}^{(1)}+\frac{1}{\left(16 \pi^{2}\right)^{2}} \beta_{\lambda}^{(2)}
$$

where the one loop contribution is given as,

$$
\beta_{\lambda}^{(1)}=12 \lambda^{2}-\left(\frac{9}{5} g_{1}^{2}+9 g_{2}^{2}\right) \lambda+\frac{9}{4}\left(\frac{3}{5} g_{1}^{4}+\frac{2}{5} g_{2}^{2} g_{2}^{2}+g_{2}^{4}\right)+4 Y_{2}(S) \lambda-4 H(S),
$$

with

$$
H(S)=\operatorname{Tr}\left(3\left(Y_{u}^{\dagger} Y_{u}\right)^{2}+3\left(Y_{d}^{\dagger} Y_{d}\right)^{2}+\left(Y_{e}^{\dagger} Y_{e}\right)^{2}\right)
$$

and the two loop contribution is given as:

$$
\begin{aligned}
\beta_{\lambda}^{(2)} & =-78 \lambda^{3}+18\left(\frac{3}{5} g_{1}^{2}+3 g_{2}^{2}\right) \lambda^{2}-\left[\left(\frac{313}{8}-10 n_{g}\right) g_{2}^{4}-\frac{117}{20} g_{1}^{2} g_{2}^{2}-\left(\frac{687}{200}+2 n_{g}\right) g_{1}^{4}\right] \lambda \\
& +\left(\frac{497}{8}-8 n_{g}\right) g_{2}^{3}-\frac{3}{5}\left(\frac{97}{24}+\frac{8}{3} n_{g}\right) g_{1}^{2} g_{2}^{4}-\frac{9}{25}\left(\frac{239}{24}+\frac{40}{9} n_{g}\right) g_{1}^{4} g_{2}^{2}-\frac{27}{125}\left(\frac{59}{24}+\frac{40}{9} n_{g}\right) g_{1}^{6} \\
& -64 g_{3}^{2} \operatorname{Tr}\left(\left(Y_{u}^{\dagger} Y_{u}\right)^{2}+\left(Y_{d}^{\dagger} Y_{d}\right)^{2}\right)-\frac{8}{5} g_{1}^{2} \operatorname{Tr}\left(2\left(Y_{u}^{\dagger} Y_{u}\right)^{2}-\left(Y_{d}^{\dagger} Y_{d}\right)^{2}+3\left(Y_{e}^{\dagger} Y_{e}\right)^{2}\right)-\frac{3}{2} g_{2}^{4} Y_{2}(S) \\
& +10 \lambda\left[\left(\frac{17}{20} g_{1}^{2}+\frac{9}{4} g_{2}^{2}+8 g_{3}^{2}\right) \operatorname{Tr}\left(Y_{u}^{\dagger} Y_{u}\right)+\left(\frac{1}{4} g_{1}^{2}+\frac{9}{4} g_{2}^{2}+8 g_{3}^{2}\right) \operatorname{Tr}\left(Y_{d}^{\dagger} Y_{d}\right)+\frac{3}{4}\left(g_{1}^{2}+g_{2}^{2}\right) \operatorname{Tr}\left(Y_{e}^{\dagger} Y_{e}\right)\right] \\
& +\frac{3}{5} g_{1}^{2}\left[\left(-\frac{57}{10} g_{1}^{2}+21 g_{2}^{2}\right) \operatorname{Tr}\left(Y_{u}^{\dagger} Y_{u}\right)+\left(\frac{3}{2} g_{1}^{2}+9 g_{2}^{2}\right) \operatorname{Tr}\left(Y_{d}^{\dagger} Y_{d}\right)+\left(-\frac{15}{2} g_{1}^{2}+11 g_{2}^{2}\right) \operatorname{Tr}\left(Y_{e}^{\dagger} Y_{e}\right)\right] \\
& -24 \lambda^{2} Y_{2}(S)-\lambda H(S)-42 \lambda \operatorname{Tr}\left(Y_{u}^{\dagger} Y_{u} Y_{d}^{\dagger} Y_{d}\right)+20 \operatorname{Tr}\left(3\left(Y_{u}^{\dagger} Y_{u}\right)^{3}+3\left(Y_{d}^{\dagger} Y_{d}\right)^{3}+\left(Y_{e}^{\dagger} Y_{e}\right)^{3}\right) \\
& -12 \operatorname{Tr}\left\{Y_{u}^{\dagger} Y_{u}\left(Y_{u}^{\dagger} Y_{u}+Y_{d}^{\dagger} Y_{d}\right) Y_{d}^{\dagger} Y_{d}\right\}
\end{aligned}
$$

where $n_{g}$ is the number of generation of fermions in SM.

Open Access. This article is distributed under the terms of the Creative Commons Attribution License (CC-BY 4.0), which permits any use, distribution and reproduction in any medium, provided the original author(s) and source are credited.

\section{References}

[1] C. Csáki and P. Tanedo, Beyond the Standard Model, in Proceedings, 2013 European School of High-Energy Physics (ESHEP 2013): Paradfurdo, Hungary, June 5-18, 2013, pp. 169-268, arXiv: 1602.04228 [https://doi.org/10.5170/CERN-2015-004.169] [INSPIRE].

[2] P.W. Graham, D.E. Kaplan and S. Rajendran, Cosmological Relaxation of the Electroweak Scale, Phys. Rev. Lett. 115 (2015) 221801 [arXiv: 1504.07551] [INSPIRE].

[3] J.R. Espinosa, C. Grojean, G. Panico, A. Pomarol, O. Pujolàs and G. Servant, Cosmological Higgs-Axion Interplay for a Naturally Small Electroweak Scale, Phys. Rev. Lett. 115 (2015) 251803 [arXiv: 1506.09217] [INSPIRE].

[4] K. Choi and S.H. Im, Realizing the relaxion from multiple axions and its UV completion with high scale supersymmetry, JHEP 01 (2016) 149 [arXiv:1511.00132] [INSPIRE]. 
[5] D.E. Kaplan and R. Rattazzi, Large field excursions and approximate discrete symmetries from a clockwork axion, Phys. Rev. D 93 (2016) 085007 [arXiv: 1511.01827] [InSPIRE].

[6] N. Arkani-Hamed, S. Dimopoulos, G.F. Giudice and A. Romanino, Aspects of split supersymmetry, Nucl. Phys. B 709 (2005) 3 [hep-ph/0409232] [INSPIRE].

[7] G.F. Giudice and A. Romanino, Split supersymmetry, Nucl. Phys. B 699 (2004) 65 [Erratum ibid. B 706 (2005) 487] [hep-ph/0406088] [INSPIRE].

[8] N. Arkani-Hamed and S. Dimopoulos, Supersymmetric unification without low energy supersymmetry and signatures for fine-tuning at the LHC, JHEP 06 (2005) 073 [hep-th/0405159] [INSPIRE].

[9] ATLAS collaboration, Search for heavy long-lived charged R-hadrons with the ATLAS detector in 3.2 $\mathrm{fb}^{-1}$ of proton-proton collision data at $\sqrt{s}=13 \mathrm{TeV}$, Phys. Lett. B 760 (2016) 647 [arXiv: 1606.05129$]$ [INSPIRE].

[10] CMS collaboration, Search for long-lived charged particles in proton-proton collisions at $\sqrt{s}=13$ TeV, Phys. Rev. D 94 (2016) 112004 [arXiv:1609.08382] [InSPIRE].

[11] C. Liu and Z.-h. Zhao, $\theta_{13}$ and the Higgs mass from high scale supersymmetry, Commun. Theor. Phys. 59 (2013) 467 [arXiv:1205.3849] [InSPIRE].

[12] F. Hartmann, W. Kilian and K. Schnitter, Multiple Scales in Pati-Salam Unification Models, JHEP 05 (2014) 064 [arXiv: 1401.7891] [INSPIRE].

[13] T.G. Rizzo, Desert guts and new light degrees of freedom, Phys. Rev. D 45 (1992) 3903 [INSPIRE].

[14] D. Choudhury, T.M.P. Tait and C.E.M. Wagner, Beautiful mirrors and precision electroweak data, Phys. Rev. D 65 (2002) 053002 [hep-ph/0109097] [INSPIRE].

[15] D.E. Morrissey and C.E.M. Wagner, Beautiful mirrors, unification of couplings and collider phenomenology, Phys. Rev. D 69 (2004) 053001 [hep-ph/0308001] [INSPIRE].

[16] V. Barger, C.-W. Chiang, J. Jiang and T. Li, Axion models with high-scale supersymmetry breaking, Nucl. Phys. B 705 (2005) 71 [hep-ph/0410252] [INSPIRE].

[17] D. Emmanuel-Costa and R. Gonzalez Felipe, Minimal string-scale unification of gauge couplings, Phys. Lett. B 623 (2005) 111 [hep-ph/0505257] [INSPIRE].

[18] V. Barger, J. Jiang, P. Langacker and T. Li, String scale gauge coupling unification with vector-like exotics and non-canonical $\mathrm{U}(1)(Y)$ normalization, Int. J. Mod. Phys. A 22 (2007) 6203 [hep-ph/0612206] [INSPIRE].

[19] V. Barger, N.G. Deshpande, J. Jiang, P. Langacker and T. Li, Implications of Canonical Gauge Coupling Unification in High-Scale Supersymmetry Breaking, Nucl. Phys. B 793 (2008) 307 [hep-ph/0701136] [INSPIRE].

[20] L. Calibbi, L. Ferretti, A. Romanino and R. Ziegler, Gauge coupling unification, the GUT scale, and magic fields, Phys. Lett. B 672 (2009) 152 [arXiv:0812.0342] [INSPIRE].

[21] I. Donkin and A. Hebecker, Precision Gauge Unification from Extra Yukawa Couplings, JHEP 09 (2010) 044 [arXiv: 1007.3990] [INSPIRE].

[22] L.J. Hall and Y. Nomura, A Finely-Predicted Higgs Boson Mass from A Finely-Tuned Weak Scale, JHEP 03 (2010) 076 [arXiv:0910.2235] [INSPIRE].

[23] R. Dermisek, Unification of gauge couplings in the standard model with extra vectorlike families, Phys. Rev. D 87 (2013) 055008 [arXiv:1212.3035] [INSPIRE]. 
[24] L.-F. Li and F. Wu, Coupling constant unification in extensions of standard model, Int. J. Mod. Phys. A 19 (2004) 3217 [hep-ph/0304238] [InSPIRE].

[25] R. Shrock, Variants of the Standard Model with Electroweak-Singlet Quarks, Phys. Rev. D 78 (2008) 076009 [arXiv:0809.0087] [INSPIRE].

[26] I. Dorsner and P. Fileviez Perez, Unification without supersymmetry: Neutrino mass, proton decay and light leptoquarks, Nucl. Phys. B 723 (2005) 53 [hep-ph/0504276] [INSPIRE].

[27] J.L. Chkareuli, I.G. Gogoladze and A.B. Kobakhidze, Natural nonSUSY SU(N) GUTs, Phys. Lett. B 340 (1994) 63 [INSPIRE].

[28] I. Gogoladze, B. He and Q. Shafi, New Fermions at the LHC and Mass of the Higgs Boson, Phys. Lett. B 690 (2010) 495 [arXiv:1004.4217] [InSPIRE].

[29] P. Fileviez Perez, Unification with and without Supersymmetry: Adjoint SU(5), in SUSY 2007 Proceedings, 15th International Conference on Supersymmetry and Unification of Fundamental Interactions, July 26 - August 1, 2007, Karlsruhe, Germany, pp. 678-681, arXiv:0710.1321 [INSPIRE].

[30] R. Dermisek, Insensitive Unification of Gauge Couplings, Phys. Lett. B 713 (2012) 469 [arXiv:1204.6533] [INSPIRE].

[31] I. Dorsner, S. Fajfer and I. Mustac, Light vector-like fermions in a minimal SU(5) setup, Phys. Rev. D 89 (2014) 115004 [arXiv: 1401.6870] [INSPIRE].

[32] M.-L. Xiao and J.-H. Yu, Stabilizing electroweak vacuum in a vectorlike fermion model, Phys. Rev. D 90 (2014) 014007 [arXiv: 1404.0681] [InSPIRE].

[33] F. del Aguila, J. de Blas and M. Pérez-Victoria, Effects of new leptons in Electroweak Precision Data, Phys. Rev. D 78 (2008) 013010 [arXiv:0803.4008] [InSPIRE].

[34] J.A. Aguilar-Saavedra, R. Benbrik, S. Heinemeyer and M. Pérez-Victoria, Handbook of vectorlike quarks: Mixing and single production, Phys. Rev. D 88 (2013) 094010 [arXiv:1306.0572] [INSPIRE].

[35] K. Ishiwata, Z. Ligeti and M.B. Wise, New Vector-Like Fermions and Flavor Physics, JHEP 10 (2015) 027 [arXiv: 1506.03484] [INSPIRE].

[36] F. del Aguila, M. Pérez-Victoria and J. Santiago, Observable contributions of new exotic quarks to quark mixing, JHEP 09 (2000) 011 [hep-ph/0007316] [INSPIRE].

[37] N. Bizot and M. Frigerio, Fermionic extensions of the Standard Model in light of the Higgs couplings, JHEP 01 (2016) 036 [arXiv: 1508.01645] [INSPIRE].

[38] L. Lavoura and J.P. Silva, The oblique corrections from vector-like singlet and doublet quarks, Phys. Rev. D 47 (1993) 2046 [InSPIRE].

[39] S.A.R. Ellis, R.M. Godbole, S. Gopalakrishna and J.D. Wells, Survey of vector-like fermion extensions of the Standard Model and their phenomenological implications, JHEP 09 (2014) 130 [arXiv: 1404.4398] [INSPIRE].

[40] G. Degrassi et al., Higgs mass and vacuum stability in the Standard Model at NNLO, JHEP 08 (2012) 098 [arXiv: 1205.6497] [INSPIRE].

[41] R. Slansky, Group Theory for Unified Model Building, Phys. Rept. 79 (1981) 1 [INSPIRE].

[42] Particle Data Group collaboration, C. Patrignani et al., Review of Particle Physics, Chin. Phys. C 40 (2016) 100001 [inSPIRE]. 
[43] A. Aranda, J.L. Diaz-Cruz and A.D. Rojas, Anomalies, $\beta$-functions and Supersymmetric Unification with Multi-Dimensional Higgs Representations, Phys. Rev. D 80 (2009) 085027 [arXiv:0907.4552] [INSPIRE].

[44] D.R.T. Jones, The Two Loop $\beta$-function for a $G_{1} \times G_{2}$ Gauge Theory, Phys. Rev. D 25 (1982) 581 [INSPIRE].

[45] H. Arason et al., Renormalization group study of the standard model and its extensions. 1. The standard model, Phys. Rev. D 46 (1992) 3945 [INSPIRE].

[46] M.-x. Luo and Y. Xiao, Two loop renormalization group equations in the standard model, Phys. Rev. Lett. 90 (2003) 011601 [hep-ph/0207271] [INSPIRE].

[47] M.-x. Luo, H.-w. Wang and Y. Xiao, Two loop renormalization group equations in general gauge field theories, Phys. Rev. D 67 (2003) 065019 [hep-ph/0211440] [InSPIRE].

[48] M.E. Machacek and M.T. Vaughn, Two Loop Renormalization Group Equations in a General Quantum Field Theory. 3. Scalar Quartic Couplings, Nucl. Phys. B 249 (1985) 70 [inSPIRE].

[49] M.E. Machacek and M.T. Vaughn, Two Loop Renormalization Group Equations in a General Quantum Field Theory. 2. Yukawa Couplings, Nucl. Phys. B 236 (1984) 221 [INSPIRE].

[50] M.E. Machacek and M.T. Vaughn, Two Loop Renormalization Group Equations in a General Quantum Field Theory. 1. Wave Function Renormalization, Nucl. Phys. B 222 (1983) 83 [INSPIRE].

[51] J. Elias-Miro, J.R. Espinosa, G.F. Giudice, G. Isidori, A. Riotto and A. Strumia, Higgs mass implications on the stability of the electroweak vacuum, Phys. Lett. B 709 (2012) 222 [arXiv:1112.3022] [INSPIRE].

[52] D. Buttazzo et al., Investigating the near-criticality of the Higgs boson, JHEP 12 (2013) 089 [arXiv: 1307.3536] [INSPIRE].

[53] Particle Data Group collaboration, K.A. Olive et al., Review of Particle Physics, Chin. Phys. C 38 (2014) 090001 [InSPIRE].

[54] ATLAS and CMS collaborations, Combined Measurement of the Higgs Boson Mass in pp Collisions at $\sqrt{s}=7$ and $8 \mathrm{TeV}$ with the ATLAS and CMS Experiments, Phys. Rev. Lett. 114 (2015) 191803 [arXiv: 1503.07589] [INSPIRE].

[55] ATLAS, CDF, CMS and D0 collaborations, First combination of Tevatron and LHC measurements of the top-quark mass, arXiv:1403.4427 [INSPIRE].

[56] S. Bethke, World Summary of $\alpha_{s}$ (2011), Nucl. Phys. Proc. Suppl. 222-224 (2012) 94 [INSPIRE].

[57] K.G. Chetyrkin and M. Steinhauser, Short distance mass of a heavy quark at order $\alpha_{s}^{3}$, Phys. Rev. Lett. 83 (1999) 4001 [hep-ph/9907509] [INSPIRE].

[58] K.G. Chetyrkin and M. Steinhauser, The relation between the MS-bar and the on-shell quark mass at order $\alpha_{s}^{3}$, Nucl. Phys. B 573 (2000) 617 [hep-ph/9911434] [INSPIRE].

[59] K. Melnikov and T.v. Ritbergen, The three loop relation between the MS-bar and the pole quark masses, Phys. Lett. B 482 (2000) 99 [hep-ph/9912391] [INSPIRE].

[60] Z.-z. Xing, H. Zhang and S. Zhou, Updated Values of Running Quark and Lepton Masses, Phys. Rev. D 77 (2008) 113016 [arXiv:0712.1419] [InSPIRE].

[61] L. Lavoura and L. Wolfenstein, Resuscitation of minimal SO(10) grand unification, Phys. Rev. D 48 (1993) 264 [INSPIRE]. 
[62] S.A.R. Ellis and J.D. Wells, Visualizing gauge unification with high-scale thresholds, Phys. Rev. D 91 (2015) 075016 [arXiv: 1502.01362] [InSPIRE].

[63] Super-Kamiokande collaboration, V. Takhistov, Review of Nucleon Decay Searches at Super-Kamiokande, in Proceedings, 51st Rencontres de Moriond on Electroweak Interactions and Unified Theories: La Thuile, Italy, March 12-19, 2016, pp. 437-444, arXiv:1605.03235, [INSPIRE].

[64] F. Staub, SARAH, arXiv:0806.0538 [INSPIRE].

[65] C. Arina and N. Sahu, Asymmetric Inelastic Inert Doublet Dark Matter from Triplet Scalar Leptogenesis, Nucl. Phys. B 854 (2012) 666 [arXiv:1108.3967] [INSPIRE].

[66] C. Arina, R.N. Mohapatra and N. Sahu, Co-genesis of Matter and Dark Matter with Vector-like Fourth Generation Leptons, Phys. Lett. B 720 (2013) 130 [arXiv:1211.0435] [INSPIRE].

[67] R. Foot, H. Lew, X.G. He and G.C. Joshi, Seesaw Neutrino Masses Induced by a Triplet of Leptons, Z. Phys. C 44 (1989) 441 [inSPIRE].

[68] E. Ma, Pathways to naturally small neutrino masses, Phys. Rev. Lett. 81 (1998) 1171 [hep-ph/9805219] [INSPIRE].

[69] F. del Aguila and J.A. Aguilar-Saavedra, Electroweak scale seesaw and heavy Dirac neutrino signals at LHC, Phys. Lett. B 672 (2009) 158 [arXiv: 0809.2096] [INSPIRE].

[70] M. Cirelli, N. Fornengo and A. Strumia, Minimal dark matter, Nucl. Phys. B 753 (2006) 178 [hep-ph/0512090] [INSPIRE].

[71] B. Bhattacherjee, M. Ibe, K. Ichikawa, S. Matsumoto and K. Nishiyama, Wino Dark Matter and Future dSph Observations, JHEP 07 (2014) 080 [arXiv: 1405.4914] [INSPIRE].

[72] M. Cirelli, F. Sala and M. Taoso, Wino-like Minimal Dark Matter and future colliders, JHEP 10 (2014) 033 [Erratum ibid. 01 (2015) 041] [arXiv:1407.7058] [INSPIRE].

[73] T. Moroi, M. Nagai and M. Takimoto, Non-Thermal Production of Wino Dark Matter via the Decay of Long-Lived Particles, JHEP 07 (2013) 066 [arXiv: 1303.0948] [INSPIRE].

[74] R. Franceschini, T. Hambye and A. Strumia, Type-III see-saw at LHC, Phys. Rev. D 78 (2008) 033002 [arXiv:0805.1613] [INSPIRE].

[75] M. Low and L.-T. Wang, Neutralino dark matter at $14 \mathrm{TeV}$ and $100 \mathrm{TeV}$, JHEP 08 (2014) 161 [arXiv: 1404.0682] [INSPIRE].

[76] Y. Kats and M.J. Strassler, Probing Colored Particles with Photons, Leptons and Jets, JHEP 11 (2012) 097 [Erratum ibid. 07 (2016) 009] [arXiv:1204.1119] [INSPIRE].

[77] Y. Kats and M.D. Schwartz, Annihilation decays of bound states at the LHC, JHEP 04 (2010) 016 [arXiv:0912.0526] [INSPIRE].

[78] A.D. Martin, W.J. Stirling, R.S. Thorne and G. Watt, Parton distributions for the LHC, Eur. Phys. J. C 63 (2009) 189 [arXiv:0901.0002] [INSPIRE].

[79] J.E. Younkin and S.P. Martin, QCD corrections to stoponium production at hadron colliders, Phys. Rev. D 81 (2010) 055006 [arXiv:0912.4813] [InSPIRE].

[80] A. Pineda and F.J. Yndurain, Calculation of quarkonium spectrum and $m(b), m(c)$ to order alpha-S ${ }^{4}$, Phys. Rev. D 58 (1998) 094022 [hep-ph/9711287] [INSPIRE].

[81] V.D. Barger et al., Superheavy Quarkonium Production and Decays: A New Higgs Signal, Phys. Rev. D 35 (1987) 3366 [Erratum ibid. D 38 (1988) 1632] [INSPIRE]. 
[82] ATLAS collaboration, Search for scalar diphoton resonances with $15.4 \mathrm{fb}^{-1}$ of data collected at $\sqrt{s}=13 \mathrm{TeV}$ in 2015 and 2016 with the ATLAS detector, ATLAS-CONF-2016-059 [INSPIRE].

[83] CMS collaboration, Search for high-mass diphoton resonances in proton-proton collisions at $13 \mathrm{TeV}$ and combination with $8 \mathrm{TeV}$ search, Phys. Lett. B 767 (2017) 147 [arXiv: 1609.02507] [INSPIRE].

[84] CMS collaboration, Search for narrow resonances in dijet final states at $\sqrt{s}=8 \mathrm{TeV}$ with the novel CMS technique of data scouting, Phys. Rev. Lett. 117 (2016) 031802 [arXiv: 1604.08907] [INSPIRE].

[85] ATLAS collaboration, Search for new phenomena in the dijet mass distribution using $p-p$ collision data at $\sqrt{s}=8 \mathrm{TeV}$ with the ATLAS detector, Phys. Rev. D 91 (2015) 052007 [arXiv:1407.1376] [INSPIRE].

[86] ATLAS collaboration, Search for new phenomena in dijet events using $37 \mathrm{fb}^{-1}$ of pp collision data collected at $\sqrt{s}=13 \mathrm{TeV}$ with the ATLAS detector, Phys. Rev. D 96 (2017) 052004 [arXiv: 1703.09127] [INSPIRE].

[87] CMS collaboration, Searches for dijet resonances in pp collisions at $\sqrt{s}=13 \mathrm{TeV}$ using data collected in 2016., CMS-PAS-EXO-16-056 [INSPIRE].

[88] ATLAS collaboration, Search for New Phenomena in Dijet Events with the ATLAS Detector at $\sqrt{s}=13 \mathrm{TeV}$ with 2015 and 2016 data, ATLAS-CONF-2016-069 [INSPIRE].

[89] CMS collaboration, Search for dijet resonances in proton-proton collisions at $\sqrt{s}=13 \mathrm{TeV}$ and constraints on dark matter and other models, Phys. Lett. B 769 (2017) 520 [Erratum ibid. B 772 (2017) 882] [arXiv: 1611.03568] [INSPIRE].

[90] F. Yu, Di-jet resonances at future hadron colliders: A snowmass whitepaper, arXiv:1308.1077 [INSPIRE].

[91] ATLAS collaboration, Combination of searches for $W W, W Z$ and $Z Z$ resonances in $p p$ collisions at $\sqrt{s}=8 \mathrm{TeV}$ with the ATLAS detector, Phys. Lett. B 755 (2016) 285 [arXiv: 1512.05099] [INSPIRE].

[92] ATLAS collaboration, Search for high-mass diphoton resonances in pp collisions at $\sqrt{s}=8 \mathrm{TeV}$ with the ATLAS detector, Phys. Rev. D 92 (2015) 032004 [arXiv:1504.05511] [INSPIRE].

[93] CMS collaboration, Search for high-mass resonances in the $\mathrm{Z}(\mathrm{q} \overline{\mathrm{q}}) \gamma$ final state at $\sqrt{s}=8 \mathrm{TeV}$, CMS-PAS-EXO-16-025 [inSPIRE].

[94] ATLAS collaboration, Search for new resonances in $W \gamma$ and $Z \gamma$ final states in pp collisions at $\sqrt{s}=8 \mathrm{TeV}$ with the ATLAS detector, Phys. Lett. B 738 (2014) 428 [arXiv:1407.8150] [INSPIRE].

[95] CMS collaboration, Search for high-mass $Z \gamma$ resonances in $\mathrm{e}^{+} \mathrm{e}^{-} \gamma$ and $\mu^{+} \mu^{-} \gamma$ final states in proton-proton collisions at $\sqrt{s}=8$ and 13 TeV, JHEP 01 (2017) 076 [arXiv:1610.02960] [INSPIRE].

[96] ATLAS collaboration, Searches for heavy $Z Z$ and $Z W$ resonances in the llqq and vvqq final states in pp collisions at $\sqrt{s}=13 \mathrm{TeV}$ with the ATLAS detector, ATLAS-CONF-2016-082 [INSPIRE].

[97] G. Cacciapaglia, A. Deandrea, N. Gaur, D. Harada, Y. Okada and L. Panizzi, Interplay of vector-like top partner multiplets in a realistic mixing set-up, JHEP 09 (2015) 012 [arXiv: 1502.00370] [INSPIRE]. 
[98] G. Cacciapaglia, A. Deandrea, D. Harada and Y. Okada, Bounds and Decays of New Heavy Vector-like Top Partners, JHEP 11 (2010) 159 [arXiv:1007.2933] [INSPIRE].

[99] S. Gopalakrishna, T. Mandal, S. Mitra and R. Tibrewala, LHC Signatures of a Vector-like b', Phys. Rev. D 84 (2011) 055001 [arXiv: 1107.4306] [INSPIRE].

[100] K. Fujikawa, A vector-like extension of the standard model, Prog. Theor. Phys. 92 (1994) 1149 [hep-ph/9411258] [INSPIRE].

[101] S. Dawson and E. Furlan, A Higgs Conundrum with Vector Fermions, Phys. Rev. D 86 (2012) 015021 [arXiv: 1205.4733] [INSPIRE].

[102] S. Fajfer, A. Greljo, J.F. Kamenik and I. Mustac, Light Higgs and Vector-like Quarks without Prejudice, JHEP 07 (2013) 155 [arXiv:1304.4219] [INSPIRE].

[103] A. Atre et al., Model-Independent Searches for New Quarks at the LHC, JHEP 08 (2011) 080 [arXiv: 1102.1987] [INSPIRE].

[104] M.E. Peskin and T. Takeuchi, A new constraint on a strongly interacting Higgs sector, Phys. Rev. Lett. 65 (1990) 964 [INSPIRE].

[105] A. Angelescu, A. Djouadi and G. Moreau, Vector-like top/bottom quark partners and Higgs physics at the LHC, Eur. Phys. J. C 76 (2016) 99 [arXiv:1510.07527] [inSPIRE].

[106] J.A. Aguilar-Saavedra, Identifying top partners at LHC, JHEP 11 (2009) 030 [arXiv: 0907.3155] [INSPIRE].

[107] R. Contino and G. Servant, Discovering the top partners at the LHC using same-sign dilepton final states, JHEP 06 (2008) 026 [arXiv:0801.1679] [INSPIRE].

[108] CMS collaboration, Inclusive search for a vector-like $T$ quark with charge $\frac{2}{3}$ in pp collisions at $\sqrt{s}=8 \mathrm{TeV}$, Phys. Lett. B 729 (2014) 149 [arXiv:1311.7667] [INSPIRE].

[109] CMS collaboration, Search for vector-like charge $2 / 3$ T quarks in proton-proton collisions at $\sqrt{s}=8$ TeV, Phys. Rev. D 93 (2016) 012003 [arXiv:1509.04177] [InSPIRE].

[110] CMS collaboration, Search for pair-produced vectorlike $B$ quarks in proton-proton collisions at $\sqrt{s}=8 \mathrm{TeV}$, Phys. Rev. D 93 (2016) 112009 [arXiv: 1507.07129] [InSPIRE].

[111] ATLAS collaboration, Search for vector-like B quarks in events with one isolated lepton, missing transverse momentum and jets at $\sqrt{s}=8 \mathrm{TeV}$ with the ATLAS detector, Phys. Rev. D 91 (2015) 112011 [arXiv: 1503.05425] [INSPIRE].

[112] ATLAS collaboration, Search for production of vector-like quark pairs and of four top quarks in the lepton-plus-jets final state in pp collisions at $\sqrt{s}=8 \mathrm{TeV}$ with the ATLAS detector, JHEP 08 (2015) 105 [arXiv: 1505.04306] [INSPIRE].

[113] M. Ibe, S. Matsumoto and R. Sato, Mass Splitting between Charged and Neutral Winos at Two-Loop Level, Phys. Lett. B 721 (2013) 252 [arXiv:1212.5989] [INSPIRE].

[114] A. Falkowski, D.M. Straub and A. Vicente, Vector-like leptons: Higgs decays and collider phenomenology, JHEP 05 (2014) 092 [arXiv: 1312.5329] [INSPIRE].

[115] W. Altmannshofer, M. Bauer and M. Carena, Exotic Leptons: Higgs, Flavor and Collider Phenomenology, JHEP 01 (2014) 060 [arXiv:1308.1987] [INSPIRE].

[116] N. Kumar and S.P. Martin, Vectorlike Leptons at the Large Hadron Collider, Phys. Rev. D 92 (2015) 115018 [arXiv:1510.03456] [INSPIRE].

[117] C.-H. Chen and T. Nomura, Bounds on LFV Higgs decays in a vector-like lepton model and searching for doubly charged leptons at the LHC, Eur. Phys. J. C 76 (2016) 353 [arXiv: 1602.07519] [INSPIRE]. 